ORISE-09-OEWH-0199

\title{
2008
}

Kansas City Plant

Annual IIIness and Injury

Surveillance Report

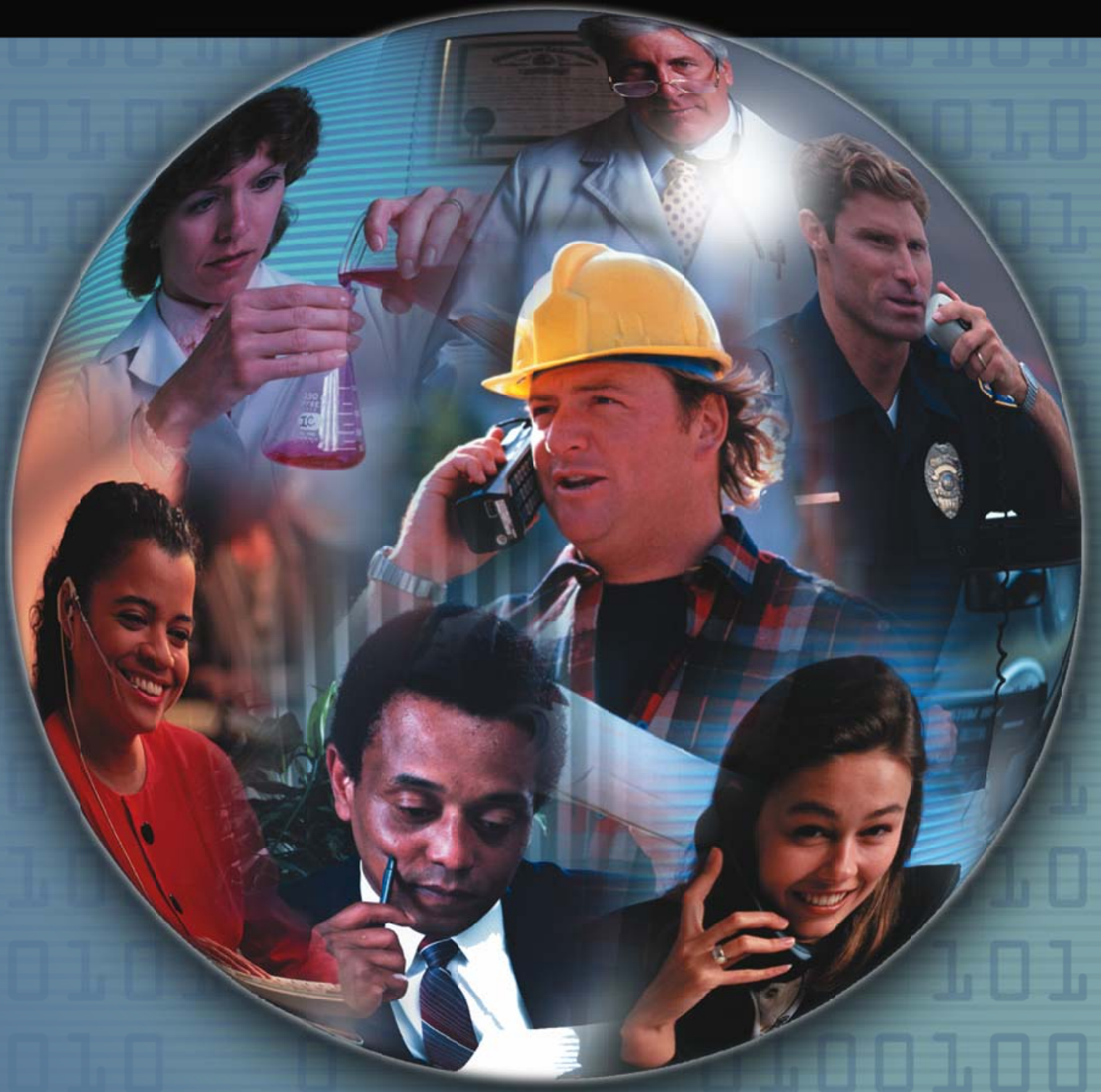




\section{Kansas City Plant 2008 Illness and Injury Surveillance Report}

Questions or comments about this report or the Illness and Injury Surveillance Program (IISP) may be directed to:

E-mail:

Dr. Cliff Strader at cliff.strader@hq.doe.gov or Dr. Bonnie Richter at bonnie.richter@hq.doe.gov

or direct letters to:

Mail Stop HS-13 / 270CC

U.S. Department of Energy

1000 Independence Avenue, S.W.

Washington, DC 20585-0270

Additional information about the Department of Energy's Office of Illness and Injury Prevention Programs, the IISP, and annual reports for DOE sites participating in this program can be found at:

http://www.hss.energy.gov/healthsafety/WSHP/epi/surv/

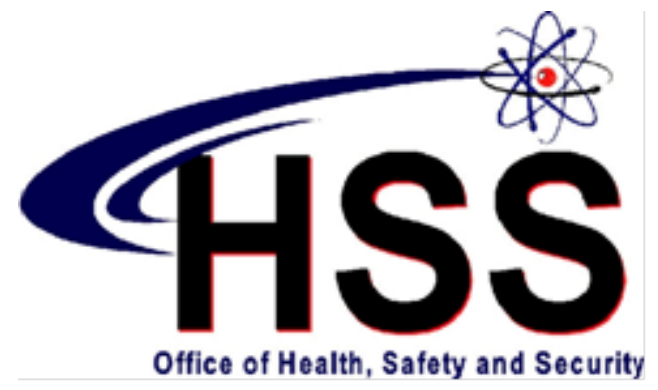

ACKNOWLEDGEMENT

LifeART images copyright 2000 Lippincott Williams \& Wilkins. All rights reserved.

This document was produced under contract number DE-AC05-06OR23100 between the

U.S. Department of Energy and Oak Ridge Associated Universities. 


\section{Kansas City Plant \\ 2008 Illness and Injury Surveillance Report}

\section{At A Glance}

A total of 2,549 Kansas City Plant employees were included in illness and injury surveillance in 2008; 652 (25 percent) women and 1,997 (75 percent) men. The ratio of women to men has been constant since 2002 and is similar to the ratio seen among all DOE IISP sites from 1998 to 2007.

Service workers had the highest absence rate; workers in the Professional group had the lowest rate among both men and women in 2008. Service workers have had the highest absence rate among men since 2006; this is the first year that women in the Service occupational group have had the highest rate since 2004 .

Women lost 2,085 calendar days due to injury and illness, about a 6 percent increase over the calendar days lost in 2007. Musculoskeletal conditions (20 percent), respiratory conditions (12 percent), and unspecified symptoms (13 percent) accounted for 45 percent of all reported diagnoses among women.

Men lost 3,655 calendar days due to injury and illness, about 7 percent more calendar days lost than in 2007. Fifty-four percent of all reported diagnoses among men were due to injuries (21 percent), musculoskeletal conditions (18 percent), and unspecified symptoms (15 percent).

The rate of all diagnoses combined increased for men and women by 12 percent and 33 percent, respectively, in 2008. The increases appear to be due to cancer and digestive system diagnoses among women, and injuries, respiratory conditions, and unspecified symptoms among men. Despite the increase noted from 2007 to 2008 , we observed no consistent change in the rates of all diagnoses combined between 2003 and 2008. 
The 2008 age-adjusted rate for all diagnoses was similar to the rates in the previous year for most occupational groups. Among women, the rate in the Service group increased sharply in 2008 but the increase was not due to any particular injury or illness category. For both men and women the rate among Security and Fire workers has steadily decreased since 2005. This decrease resulted from a decline in the number of injuries reported by men and a decline in all types of injuries and illnesses reported by women.

Six diagnoses for sentinel occupational health events were reported. Carpal tunnel syndrome accounted for half of these diagnoses and were reported by workers in the Administrative Support, Service, and Crafts groups; all were aged 50 or older.

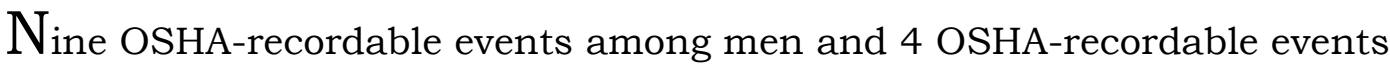
among women produced an event rate of not more than 1 per 100 workers for both men and women in 2008. Service workers had the highest rate of OSHA events among both men and women.

Musculoskeletal conditions, namely joint disorders and rheumatism, accounted for almost half (48 percent) of the OSHA diagnoses. Overexertion and strenuous movements and falls were the most frequent types of accidents.

OsHA events were responsible for 176 days lost and 298 days of restricted duty in 2008; lost days were more than 6 times higher than in 2007 and days restricted increased 77 percent. Most of the days lost were reported by a male Crafts worker for respiratory disorders. Male Technical Support, Service, Security and Fire, and Crafts workers reported 295 of the days restricted due to consequences of overexertion and strenuous movements. 
The Kansas City Plant

Work Force - 2008

The Work Force by Gender and Age ............ 1

The Work Force by Gender and Job

Category 1

\section{Number and Length of Absences}

Absence Rate by Gender and Age 2

Number of Days Absent by

Gender and Age

Absence Rate by Job Category

and Gender. .3

Average Duration of Absence by

Job Category and Gender. ... 3

\section{Diagnostic Categories}

Number of Diagnoses and Lost Calendar Days by Diagnostic Category

(Categorized by ICD-9-CM) and Gender

Common Diagnoses Among Female

Workers in 2008 . . .5

Common Diagnoses Among Male

Workers in 2008 .... 6

Number of Most Frequently Reported Diagnoses by Job Category and Gender 7

\section{Rates of Disease Occurrence}

Rates for All Illnesses and Injuries Combined by Job Category, Gender, and Age

Rates for Selected Diagnostic Categories by Job Category, Gender, and Age .8

\section{Time Trends}

Age-Adjusted Rates for All Diagnoses Combined Among Women and Men from 2003 to 2008 10
Age-Adjusted Rates for Selected Diagnostic Categories Among Women and Men from 2003 to 2008

Age-Adjusted Rates for All Diagnoses

Combined Among Women and Men by Job

Category from 2003 to 2008 ......................12

\section{Sentinel Health Events for Occupations (SHEOs)}

Characteristics of SHEOs by Gender 13

SHEO Diagnoses by Gender 13

\section{Occupational Safety and Health Administration (OSHA)-Recordable Events}

OSHA-Recordable Events by Gender and Age. 14

OSHA-Recordable Events by Job

Category and Gender 14

\section{Diagnostic and Accident Categories for OSHA-Recordable Events}

OSHA-Recordable Diagnoses by

Diagnostic Category and Gender

OSHA-Recordable Accidents by Type

and Gender.

\section{Rates of OSHA-Recordable Events}

OSHA-Recordable Rates by Age and Job

Categories Among Women, All Diagnoses

Combined

OSHA-Recordable Rates by Age and Job

Categories Among Men, All Diagnoses

Combined

Time Trends for OSHA-Recordable Events

Age-Adjusted Rates for All OSHA-Recordable Diagnoses Combined Among Women and Men by Job Category from 2003 to 2008 .

\section{Appendices}

Appendices A-W 19 
The Kansas City Plant Work Force - 2008

Figure 1. The Work Force by Gender and Age

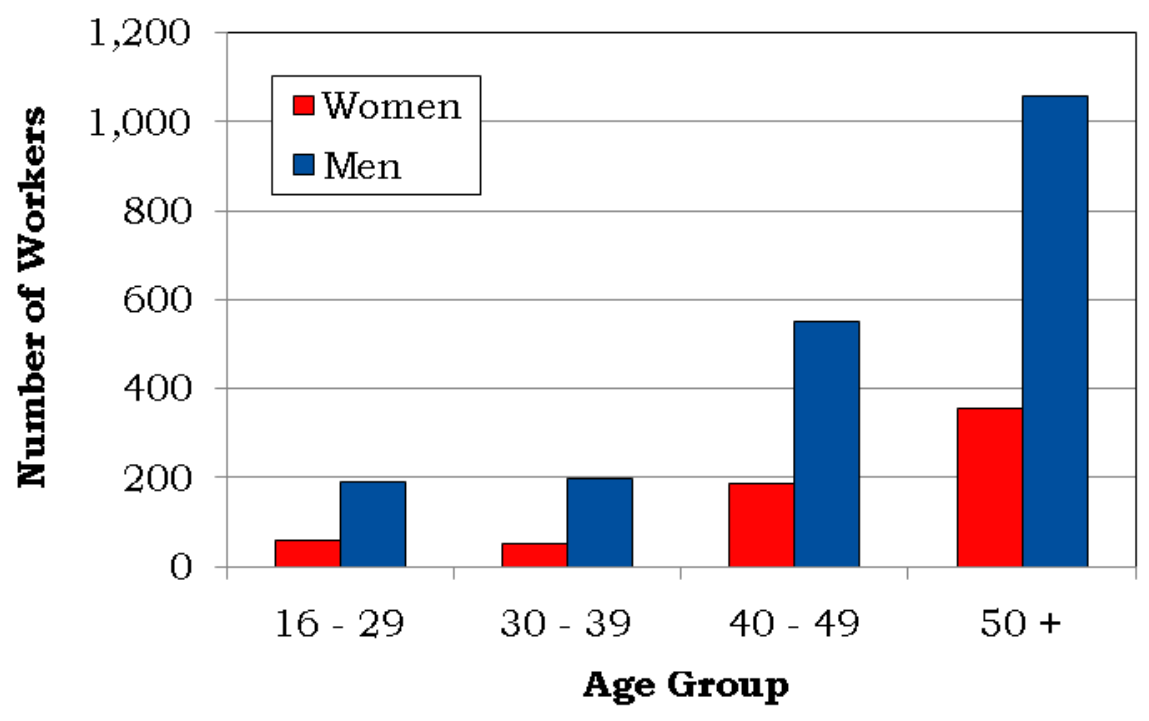

Figure 2. The Work Force by Gender and Job Category

\begin{tabular}{|l|c|c|}
\hline \multicolumn{1}{|c|}{ Job Category } & Women & M en \\
\hline \multirow{2}{*}{ Professional } & 149 & 859 \\
& $23 \%$ & $43 \%$ \\
\hline \multirow{2}{*}{ Administrative Support } & 266 & 260 \\
& $41 \%$ & $13 \%$ \\
\hline \multirow{2}{*}{ Technical Support } & 60 & 265 \\
& $9 \%$ & $14 \%$ \\
\hline \multirow{2}{*}{ Service } & 39 & 58 \\
& $6 \%$ & $3 \%$ \\
\hline \multirow{2}{*}{ Security and Fire } & 12 & 66 \\
\hline \multirow{2}{*}{ Crafts } & $2 \%$ & $3 \%$ \\
\hline \multirow{2}{*}{ Line Operators } & 18 & 325 \\
& $3 \%$ & $16 \%$ \\
\hline \multirow{2}{*}{ Total } & 108 & 164 \\
& $16 \%$ & $8 \%$ \\
\hline
\end{tabular}




\section{Number and Length of Absences}

Figure 3. Absence Rate by Gender and Age

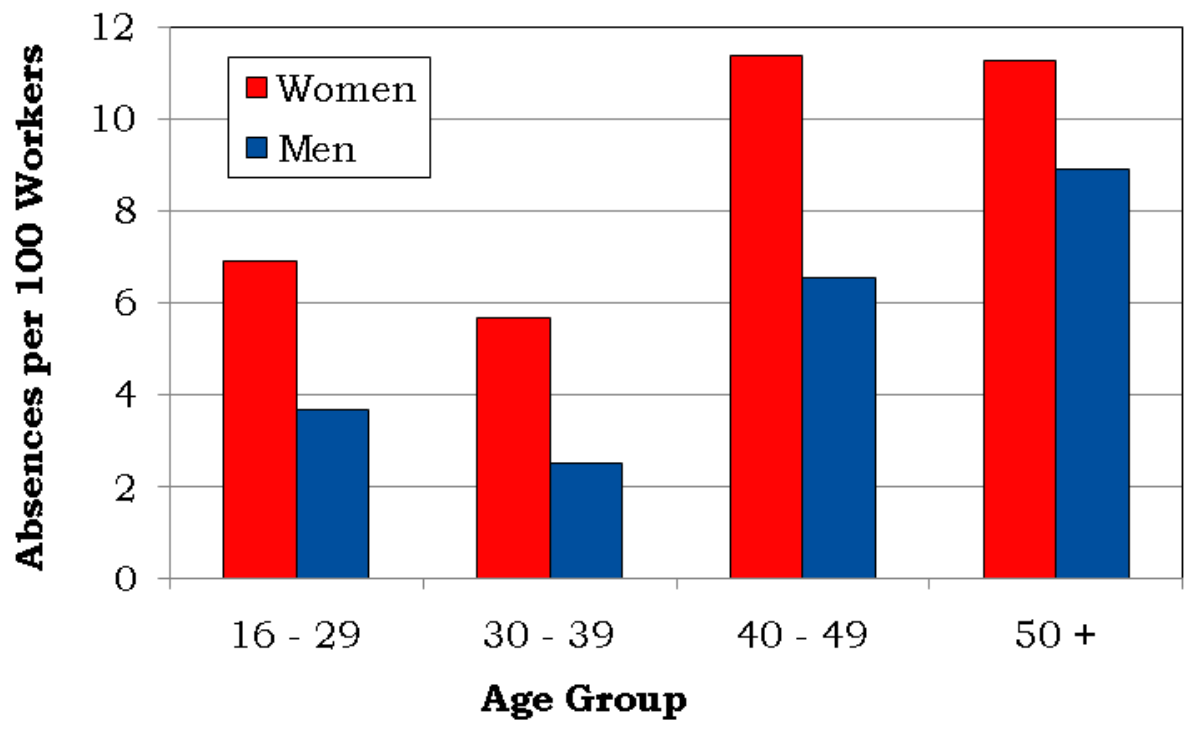

Figure 4. Number of Days Absent by Gender and Age

\begin{tabular}{|c|c|c|c|c|}
\hline \multirow{3}{*}{ Gender } & \multirow{2}{*}{ Age } & Number of & \multicolumn{2}{|c|}{ Number of Days Absent } \\
\cline { 3 - 5 } & & Absences & Total & Average \\
\hline \multirow{4}{*}{ Women } & $16-29$ & 4 & 65 & 16 \\
\cline { 2 - 5 } & $30-39$ & 3 & 24 & 8 \\
\cline { 2 - 5 } & $40-49$ & 21 & 742 & 35 \\
\cline { 2 - 5 } & $50+$ & 40 & 1,254 & 31 \\
\cline { 2 - 5 } & Total & 68 & 2,085 & 31 \\
\hline \multirow{4}{*}{ Men } & $16-29$ & 7 & 93 & 13 \\
\cline { 2 - 5 } & $30-39$ & 5 & 117 & 23 \\
\cline { 2 - 5 } & $40-49$ & 36 & 1,017 & 28 \\
\cline { 2 - 5 } & $50+$ & 94 & 2,428 & 26 \\
\cline { 2 - 5 } & Total & 142 & 3,655 & 26 \\
\hline
\end{tabular}


Figure 5. Absence Rate by Job Category and Gender

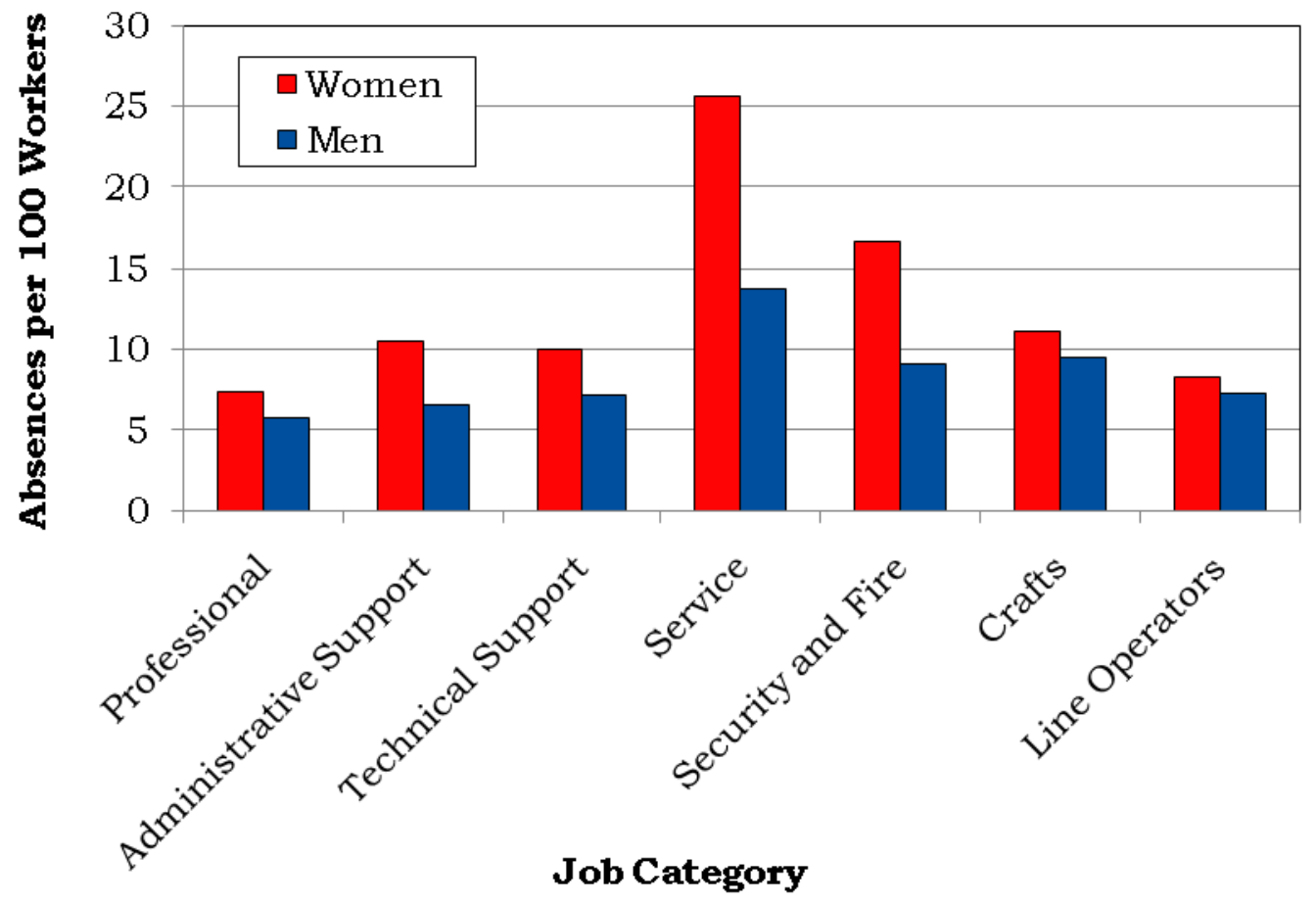

Figure 6. Average Duration of Absence by Job Category and Gender

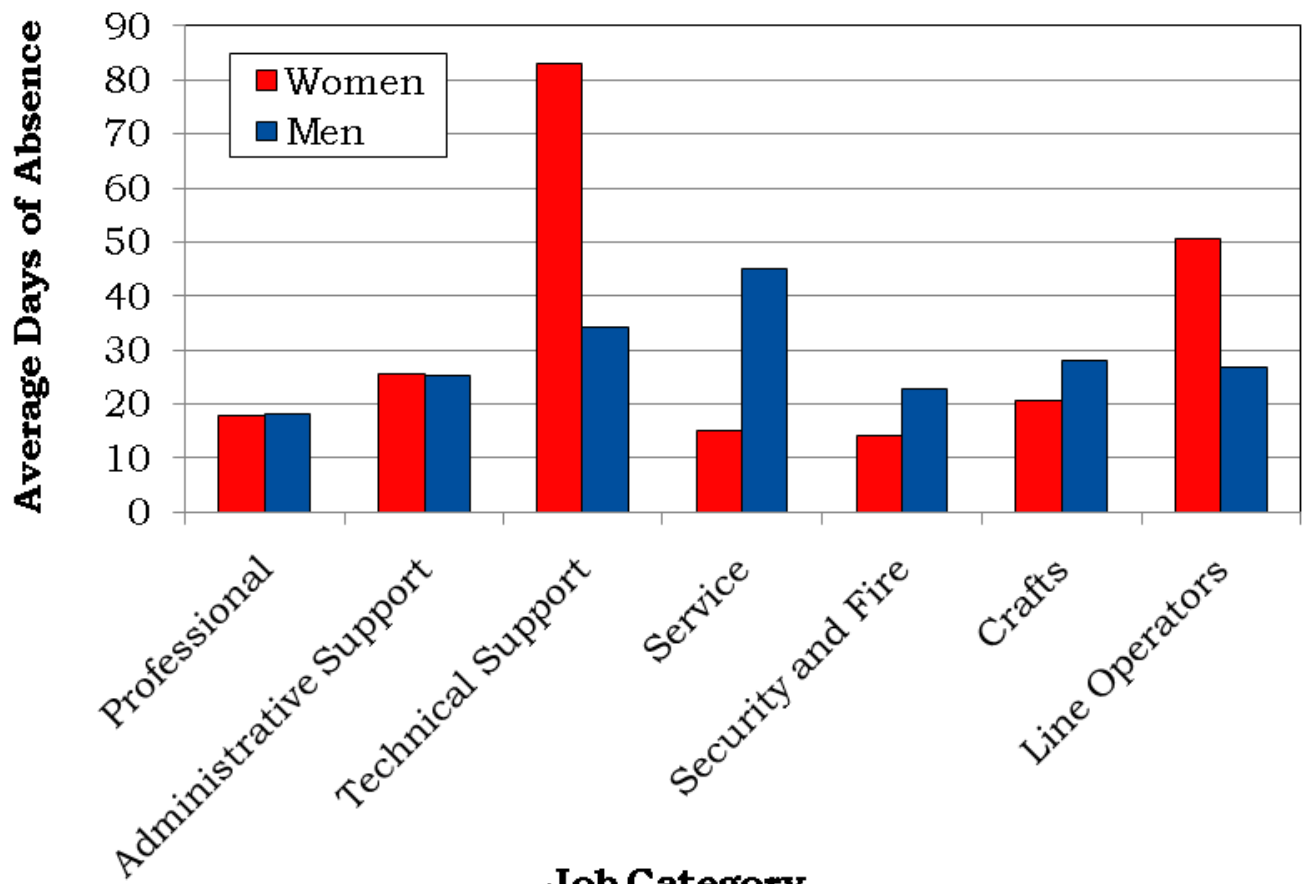

Job Category 


\section{Diagnostic Categories}

Figure 7. Number of Diagnoses and Lost Calendar Days by Diagnostic Category (Categorized by ICD-9-CM) and Gender

\begin{tabular}{|l|c|c|c|c|}
\hline \multirow{2}{*}{ Diagnostic Category } & \multicolumn{2}{|c|}{ Women } & \multicolumn{2}{c|}{ Men } \\
\cline { 2 - 5 } & $\begin{array}{c}\text { Number of } \\
\text { Diagnoses }\end{array}$ & $\begin{array}{c}\text { Number of } \\
\text { Lost } \\
\text { Calendar } \\
\text { Days }\end{array}$ & $\begin{array}{c}\text { Number of } \\
\text { Diagnoses }\end{array}$ & $\begin{array}{c}\text { Number of } \\
\text { Lost } \\
\text { Calendar } \\
\text { Days }\end{array}$ \\
\hline Benign Growths & $\mathbf{3}$ & $\mathbf{1 0 2}$ & $\mathbf{3}$ & $\mathbf{8 2}$ \\
\hline Blood & $\mathbf{1}$ & $\mathbf{4 3}$ & $\mathbf{0}$ & $\mathbf{0}$ \\
\hline Cancer & $\mathbf{9}$ & $\mathbf{3 8 0}$ & $\mathbf{1 1}$ & $\mathbf{3 2 4}$ \\
\hline Digestive & $\mathbf{7}$ & $\mathbf{1 9 6}$ & $\mathbf{2 9}$ & $\mathbf{5 4 4}$ \\
\hline Endocrine/Metabolic & $\mathbf{3}$ & $\mathbf{9 9}$ & $\mathbf{3}$ & $\mathbf{3 3}$ \\
\hline Existing Birth Condition & $\mathbf{0}$ & $\mathbf{0}$ & $\mathbf{1}$ & $\mathbf{5 0}$ \\
\hline Genitourinary & $\mathbf{9}$ & $\mathbf{2 4 4}$ & $\mathbf{4}$ & $\mathbf{1 7 7}$ \\
\hline Heart/Circulatory & $\mathbf{5}$ & $\mathbf{1 6 2}$ & $\mathbf{1 7}$ & $\mathbf{2 5 3}$ \\
\hline Infections/Parasites & $\mathbf{4}$ & $\mathbf{5 9}$ & $\mathbf{5}$ & $\mathbf{2 3 9}$ \\
\hline Injury & $\mathbf{1 0}$ & $\mathbf{4 6 0}$ & $\mathbf{4 9}$ & $\mathbf{1 , 0 3 1}$ \\
\hline Miscarriage & $\mathbf{0}$ & $\mathbf{0}$ & $\mathbf{N A}$ & NA \\
\hline Musculoskeletal & $\mathbf{2 3}$ & $\mathbf{8 3 9}$ & $\mathbf{4 2}$ & $\mathbf{1 , 2 1 7}$ \\
\hline Nervous System & $\mathbf{7}$ & $\mathbf{2 1 0}$ & $\mathbf{6}$ & $\mathbf{1 8 6}$ \\
\hline Psychological & $\mathbf{1}$ & $\mathbf{7 7}$ & $\mathbf{6}$ & $\mathbf{8 3}$ \\
\hline Respiratory & $\mathbf{1 3}$ & $\mathbf{1 2 1}$ & $\mathbf{2 1}$ & $\mathbf{3 5 2}$ \\
\hline Skin & $\mathbf{2}$ & $\mathbf{6 3}$ & $\mathbf{1}$ & $\mathbf{3 5}$ \\
\hline Unspecified Symptoms & $\mathbf{1 5}$ & $\mathbf{2 7 8}$ & $\mathbf{3 9 6}$ \\
\hline
\end{tabular}

Note: Lost calendar days for each absence are counted more than once when multiple diagnoses occur in different diagnostic categories for the same absence. 
Figure 8. Common Diagnoses Among Female Workers in 2008

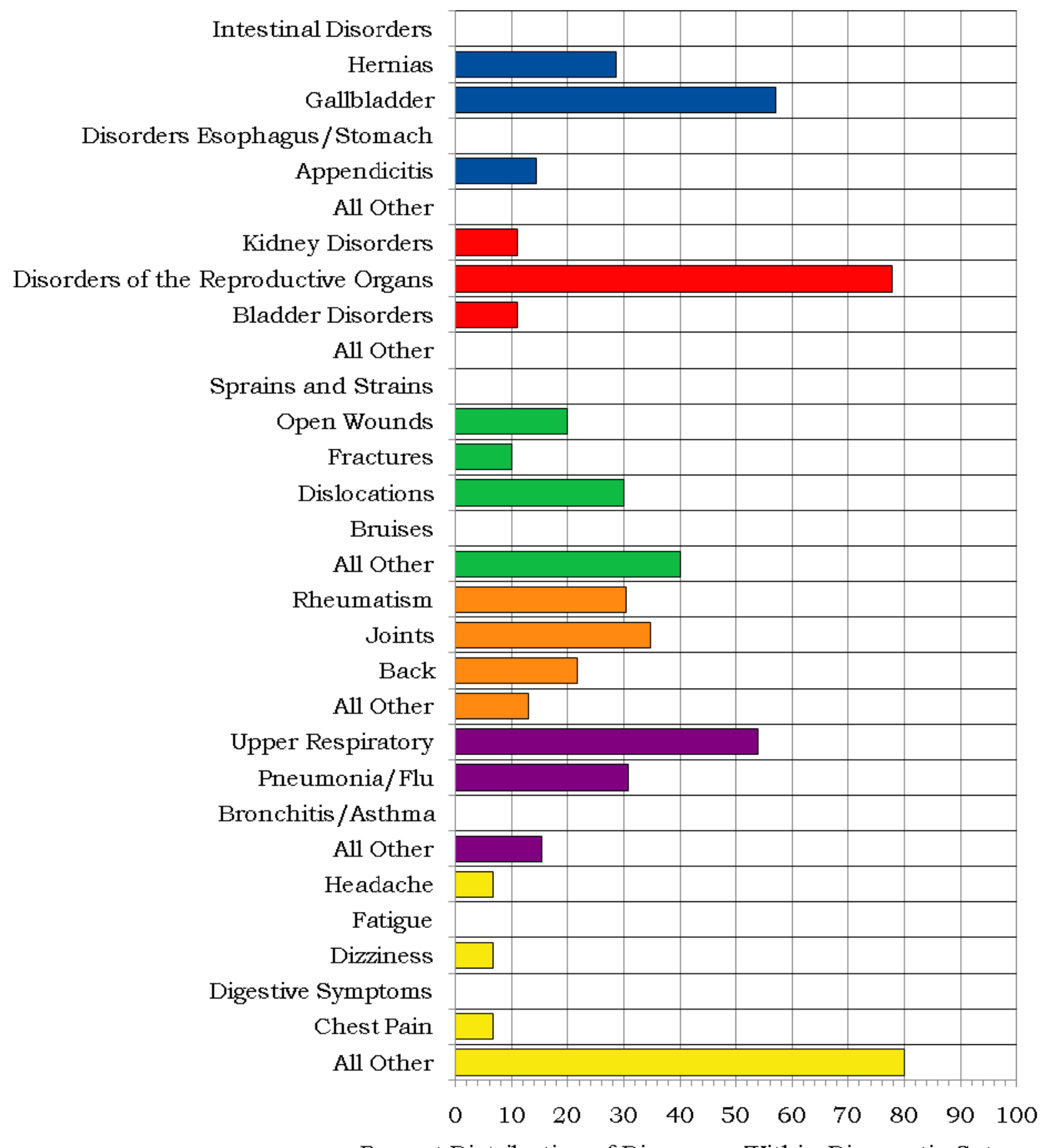

Percent Distribution of Diagnoses Within Diagnostic Category

Digestive, 7 Diagnoses

Genitourinary, 9 Diagnoses

Injury, 10 Diagnoses
Musculoskeletal, 23 Diagnoses

Respiratory, 13 Diagnoses

Unspecified Symptoms, 15 Diagnoses 
Figure 9. Common Diagnoses Among Male Workers in 2008

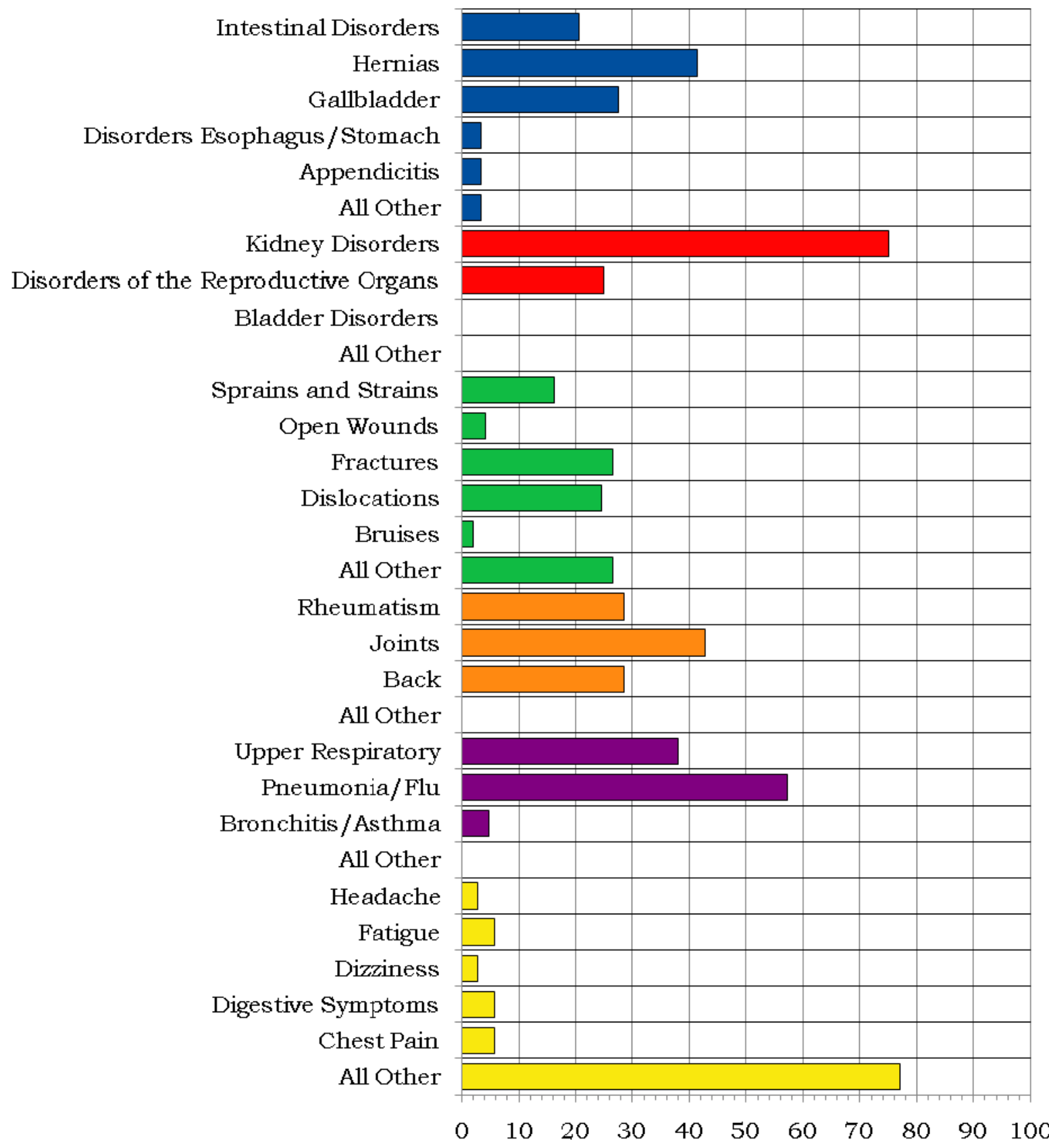

Percent Distribution of Diagnoses Within Diagnostic Category

Dige stive, 29 Diagnoses

Genitourinary, 4 Diagnoses

Injury, 49 Diagnoses
Musculoskeletal, 42 Diagnoses

Respiratory, 21 Diagnoses

Unspecified Symptoms, 35 Diagnoses 


\section{Figure 10. Number of Most Frequently Reported Diagnoses by Job Category and Gender}

\begin{tabular}{|c|c|c|c|c|}
\hline Job Category & Men & & Women & \\
\hline \multirow{3}{*}{ Professional } & Unspecified Symptoms & 16 & M usculoskeletal & 5 \\
\hline & M usculoskeletal & 14 & Respiratory & 5 \\
\hline & Digestive & 13 & Unspecified Symptoms & 2 \\
\hline \multirow{3}{*}{ Administrative Support } & Respiratory & 8 & Unspecified Symptoms & 10 \\
\hline & Musculoskeletal & 7 & M usculoskeletal & 7 \\
\hline & Injury & 5 & Cancer & 7 \\
\hline \multirow{3}{*}{ Technical Support } & Injury & 15 & M usculoskeletal & 5 \\
\hline & Respiratory & 5 & Injury & 3 \\
\hline & M usculoskeletal & 4 & Nervous System & 2 \\
\hline \multirow{4}{*}{ Service } & Injury & 8 & Injury & 3 \\
\hline & M usculoskeletal & 2 & M usculoskeletal & 2 \\
\hline & Unspecified Symptoms & 2 & Unspecified Symptoms & 2 \\
\hline & Respiratory & 2 & & \\
\hline \multirow{3}{*}{ Security and Fire } & Injury & 3 & M usculoskeletal & 2 \\
\hline & M usculoskeletal & 2 & Nervous System & 1 \\
\hline & Digestive & 1 & Infections/Parasites & 1 \\
\hline \multirow{3}{*}{ Crafts } & M usculoskeletal & 9 & Digestive & 1 \\
\hline & Digestive & 9 & Respiratory & 1 \\
\hline & Unspecified Symptoms & 9 & & \\
\hline \multirow{5}{*}{ Line Operators } & M usculoskeletal & 4 & M usculoskeletal & 2 \\
\hline & Unspecified Symptoms & 3 & Digestive & 2 \\
\hline & Heart/Circulatory & 3 & Genitourinary & 2 \\
\hline & & & Heart/Circulatory & 2 \\
\hline & & & Cancer & 2 \\
\hline
\end{tabular}




\section{Rates of Disease Occurrence}

Figure 11. Rates for All Illnesses and Injuries Combined by Job Category, Gender, and Age

\begin{tabular}{|c|c|c|c|c|}
\hline \multirow{2}{*}{$\begin{array}{c}\text { All Illnesses \& } \\
\text { Injuries Combined }\end{array}$} & \multicolumn{4}{|c|}{ Rate per 1,000} \\
\hline & Job Category & Age & Men & Women \\
\hline & \multirow{2}{*}{ Professional } & $<50$ & 81 & 57 \\
\hline & & $50+$ & 117 & 250 \\
\hline & \multirow{2}{*}{ Administrative Support } & $<50$ & 92 & 145 \\
\hline & & $50+$ & 131 & 239 \\
\hline & \multirow{2}{*}{ Technical Support } & $<50$ & 163 & 588 \\
\hline & & $50+$ & 120 & 70 \\
\hline & \multirow{2}{*}{ Service } & $<50$ & 185 & 556 \\
\hline & & $50+$ & 355 & 233 \\
\hline & \multirow{2}{*}{ Security and Fire } & $<50$ & 71 & 0 \\
\hline & & $50+$ & 105 & 800 \\
\hline & \multirow{2}{*}{ Crafts } & $<50$ & 82 & 0 \\
\hline & & $50+$ & 167 & 133 \\
\hline & \multirow{2}{*}{ Line Operators } & $<50$ & 39 & 129 \\
\hline & & $50+$ & 197 & 104 \\
\hline
\end{tabular}

Figure 12. Rates for Selected Diagnostic Categories by Job Category, Gender, and Age

\begin{tabular}{|c|c|c|c|c|}
\hline \multirow{2}{*}{ Cancer } & \multicolumn{4}{|c|}{ Rate per 1,000} \\
\hline & Job Category & Age & Men & Women \\
\hline & \multirow{2}{*}{ Professional } & $<50$ & 4 & 0 \\
\hline & & $50+$ & 10 & 0 \\
\hline & \multirow{2}{*}{ Administrative Support } & $<50$ & 0 & 8 \\
\hline & & $50+$ & 0 & 42 \\
\hline & \multirow{2}{*}{ Technical Support } & $<50$ & 0 & 0 \\
\hline & & $50+$ & 0 & 0 \\
\hline & \multirow{2}{*}{ Service } & $<50$ & 0 & 0 \\
\hline & & $50+$ & 0 & 0 \\
\hline & \multirow{2}{*}{ Security and Fire } & $<50$ & 0 & 0 \\
\hline & & $50+$ & 0 & 0 \\
\hline & \multirow{2}{*}{ Crafts } & $<50$ & 0 & 0 \\
\hline & & $50+$ & 18 & 0 \\
\hline & \multirow{2}{*}{ Line Operators } & $<50$ & 0 & 0 \\
\hline & & $50+$ & 16 & 26 \\
\hline
\end{tabular}


Figure 12. Rates for Selected Diagnostic Categories by Job Category, Gender, and Age (Continued)

\begin{tabular}{|l|l|c|c|c|}
\hline \multirow{2}{*}{ Heart/Circulatory } & \multicolumn{4}{|c|}{ Rate per 1,000 } \\
\cline { 2 - 5 } & \multicolumn{1}{|c|}{ Job Category } & Age & M en & W omen \\
\hline \multirow{2}{*}{ Profe ssional } & $<50$ & $\mathbf{4}$ & $\mathbf{0}$ \\
\cline { 2 - 5 } & $50+$ & $\mathbf{1 2}$ & $\mathbf{0}$ \\
\cline { 2 - 5 } & \multirow{2}{*}{ Administrative Support } & $<50$ & $\mathbf{1 5}$ & $\mathbf{8}$ \\
\cline { 2 - 5 } & $50+$ & $\mathbf{0}$ & $\mathbf{7}$ \\
\cline { 2 - 5 } & Technical Support & $<50$ & $\mathbf{0}$ & $\mathbf{0}$ \\
\cline { 2 - 5 } & $50+$ & $\mathbf{1 2}$ & $\mathbf{0}$ \\
\cline { 2 - 5 } & \multirow{2}{*}{ Service } & $<50$ & $\mathbf{0}$ & $\mathbf{1 1 1}$ \\
\cline { 2 - 5 } & $50+$ & $\mathbf{0}$ & $\mathbf{0}$ \\
\cline { 2 - 5 } & \multirow{2}{*}{ Security and Fire } & $<50$ & $\mathbf{0}$ & $\mathbf{0}$ \\
\cline { 2 - 5 } & $50+$ & $\mathbf{0}$ & $\mathbf{0}$ \\
\cline { 2 - 5 } & \multirow{2}{*}{ Crafts } & $<50$ & $\mathbf{0}$ & $\mathbf{0}$ \\
\cline { 2 - 5 } & $50+$ & $\mathbf{1 3}$ & $\mathbf{0}$ \\
\cline { 2 - 5 } & \multirow{2}{*}{ Line Operators } & $<50$ & $\mathbf{1 0}$ & $\mathbf{0}$ \\
\cline { 2 - 5 } & $50+$ & $\mathbf{3 3}$ & $\mathbf{2 6}$ \\
\hline
\end{tabular}

\begin{tabular}{|c|c|c|c|c|}
\hline \multirow{2}{*}{ Respiratory } & \multicolumn{4}{|c|}{ Rate per 1,000} \\
\hline & Job Category & Age & Men & Women \\
\hline \multirow{14}{*}{3} & \multirow{2}{*}{ Professional } & $<50$ & 7 & 19 \\
\hline & & $50+$ & 2 & 68 \\
\hline & \multirow{2}{*}{ Administrative Support } & $<50$ & 31 & 24 \\
\hline & & $50+$ & 31 & 21 \\
\hline & \multirow{2}{*}{ Technical Support } & $<50$ & 20 & 0 \\
\hline & & $50+$ & 18 & 0 \\
\hline & \multirow{2}{*}{ Service } & $<50$ & 37 & 0 \\
\hline & & $50+$ & 32 & 33 \\
\hline & \multirow{2}{*}{ Security and Fire } & $<50$ & 0 & 0 \\
\hline & & $50+$ & 0 & 0 \\
\hline & \multirow{2}{*}{ Crafts } & $<50$ & 0 & 0 \\
\hline & & $50+$ & 4 & 67 \\
\hline & \multirow{2}{*}{ Line Operators } & $<50$ & 0 & 0 \\
\hline & & $50+$ & 16 & 0 \\
\hline
\end{tabular}


Figure 12. Rates for Selected Diagnostic Categories by Job Category, Gender, and Age (Continued)

\begin{tabular}{|c|c|c|c|c|}
\hline \multirow{2}{*}{ Injury } & \multicolumn{4}{|c|}{ Rate per 1,000} \\
\hline & Job Category & Age & Men & Women \\
\hline & \multirow{2}{*}{ Professional } & $<50$ & 11 & 0 \\
\hline & & $50+$ & 12 & 23 \\
\hline & \multirow{2}{*}{ Administrative Support } & $<50$ & 15 & 8 \\
\hline & & $50+$ & 23 & 14 \\
\hline & \multirow{2}{*}{ Technical Support } & $<50$ & 92 & 118 \\
\hline & & $50+$ & 36 & 23 \\
\hline & \multirow{2}{*}{ Service } & $<50$ & 111 & 0 \\
\hline & & $50+$ & 161 & 100 \\
\hline & \multirow{2}{*}{ Security and Fire } & $<50$ & 36 & 0 \\
\hline & & $50+$ & 53 & 0 \\
\hline & \multirow{2}{*}{ Crafts } & $<50$ & 10 & 0 \\
\hline & & $50+$ & 26 & 0 \\
\hline & \multirow{2}{*}{ Line Operators } & $<50$ & 10 & 0 \\
\hline & & $50+$ & 0 & 0 \\
\hline
\end{tabular}

\section{Time Trends}

Figure 13. Age-Adjusted Rates for All Diagnoses Combined Among Women and Men from 2003 to 2008*

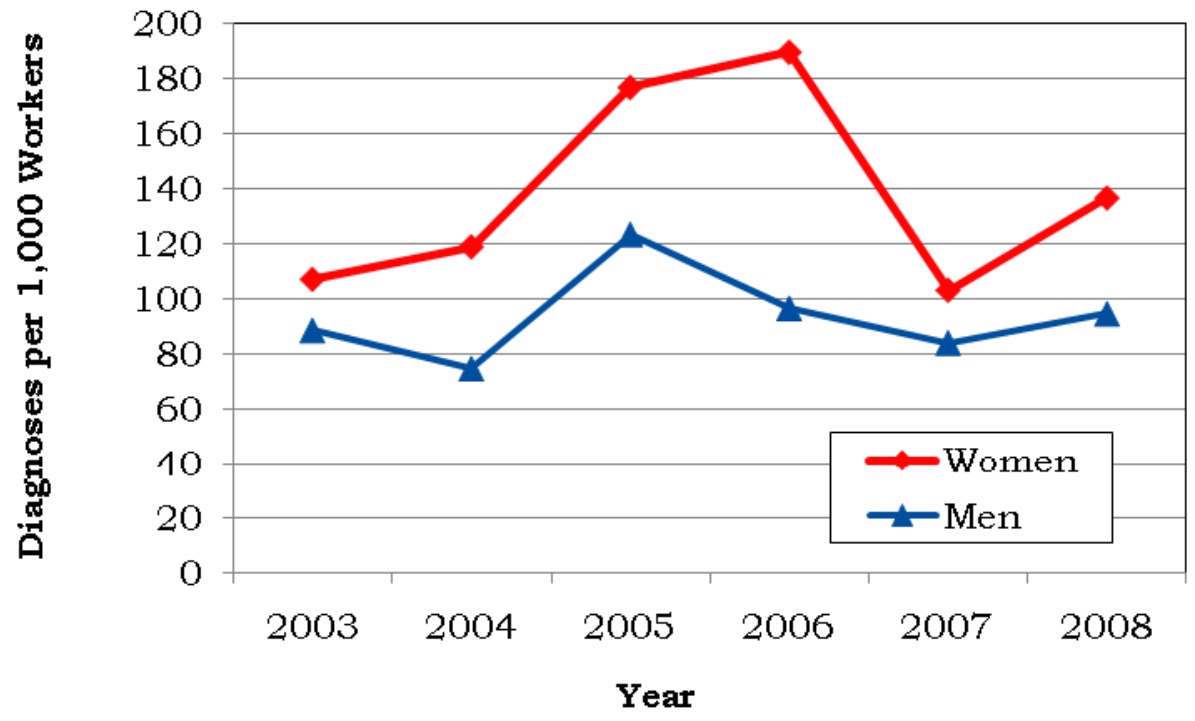

*Standardized to age distribution of 2000 U.S. population. 
Figure 14. Age-Adjusted Rates for Selected Diagnostic Categories Among Women and Men from 2003 to 2008*

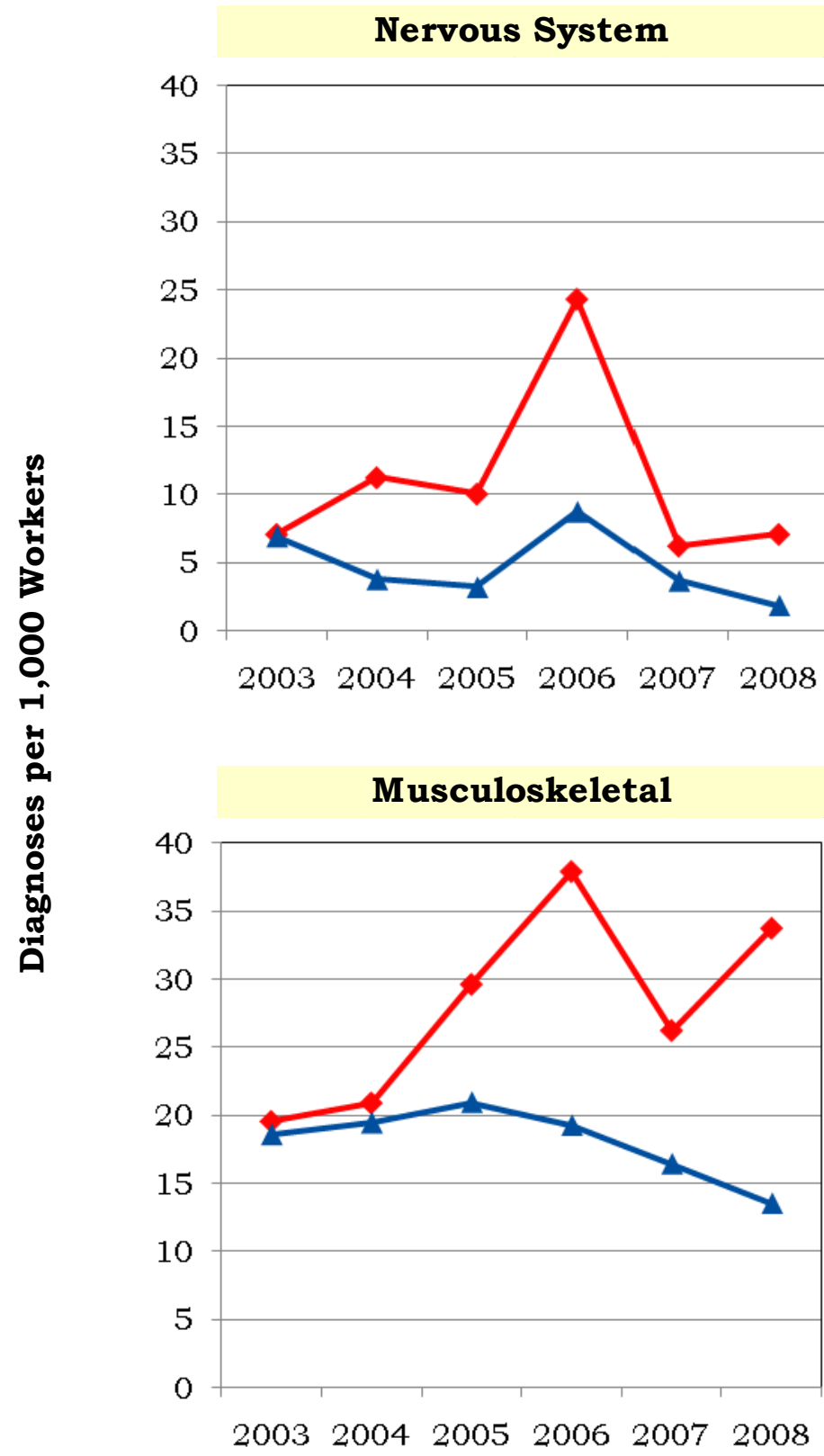

Chronic Respiratory

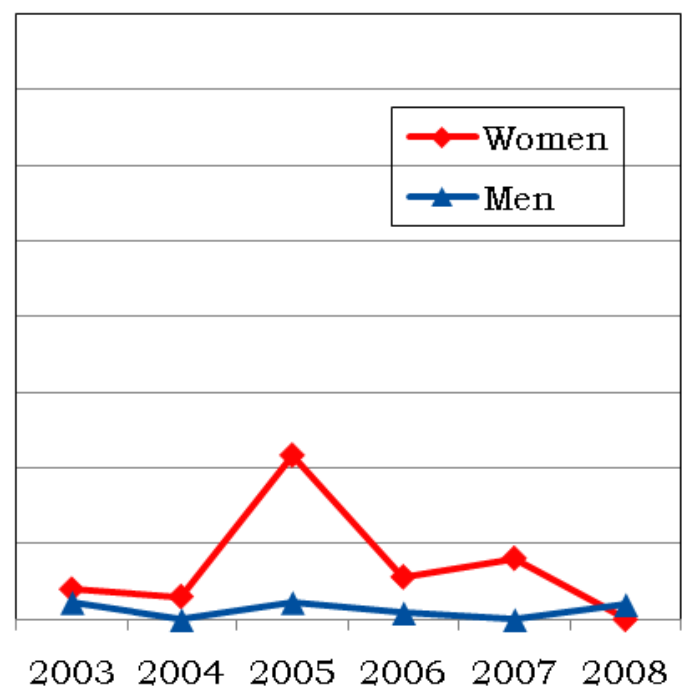

Injury

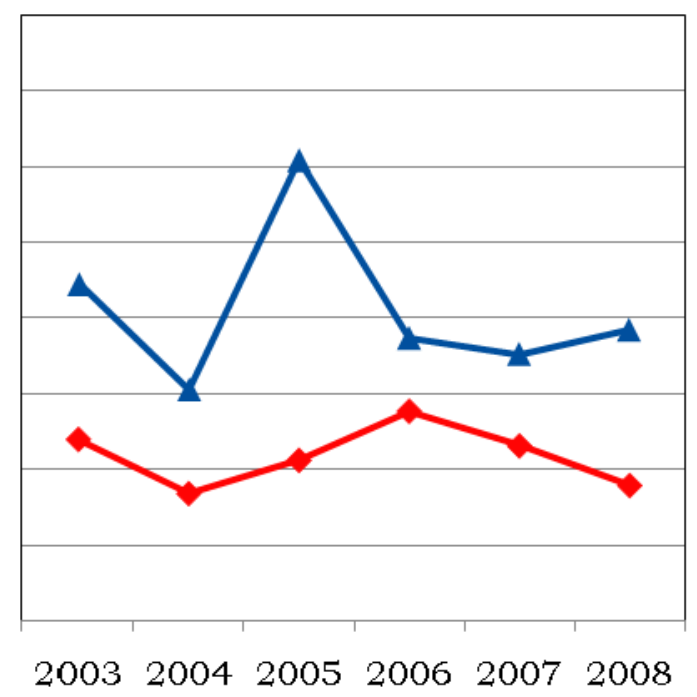

Year

*Standardized to age distribution of 2000 U.S. population. 
Figure 15. Age-Adjusted Rates for All Diagnoses Combined Among Women and Men by Job Category from 2003 to 2008*

Professional

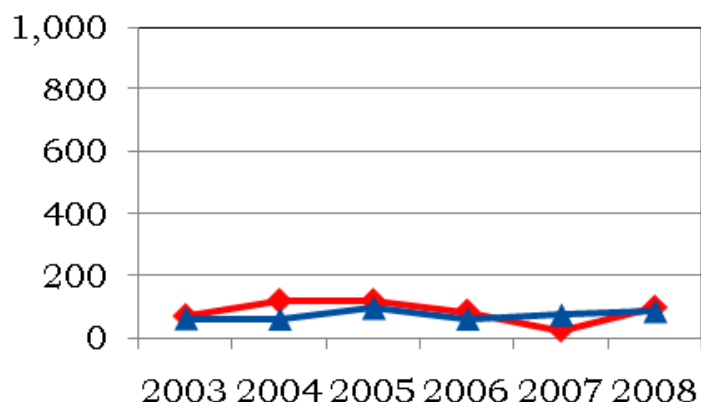

Administrative Support
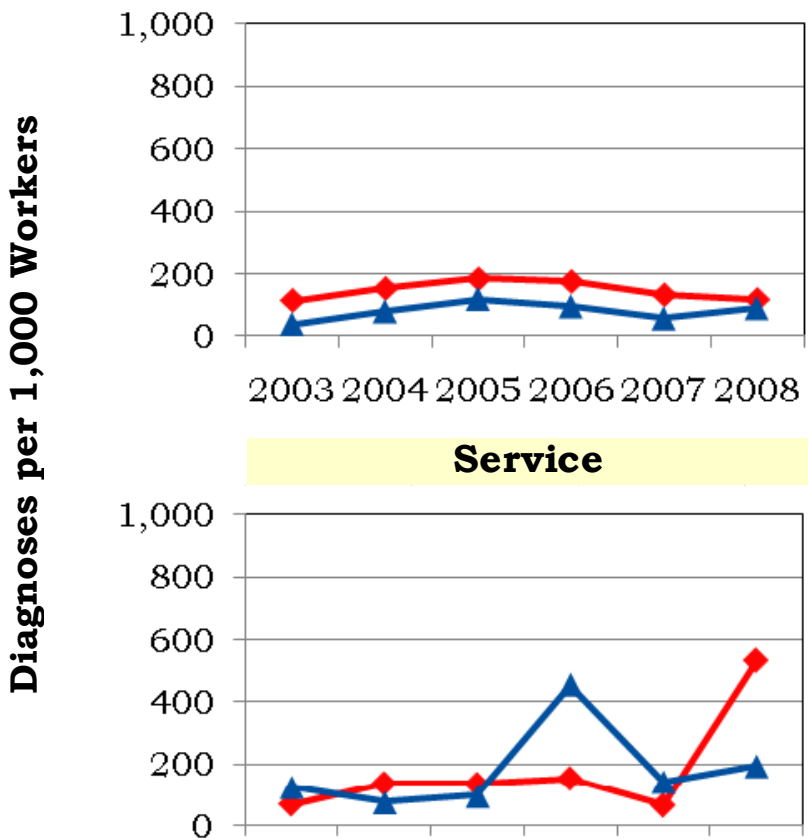

200320042005200620072008

Crafts

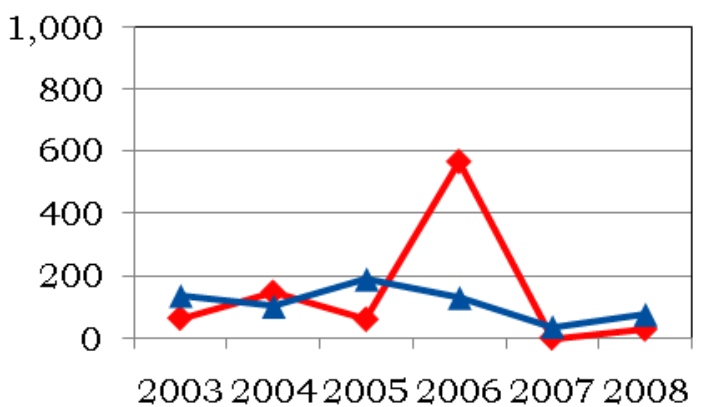

\section{Technical Support}

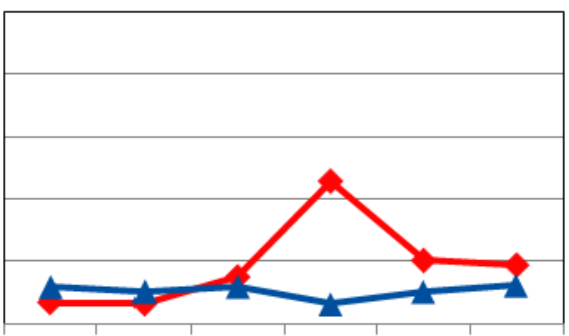

200320042005200620072008

Security and Fire

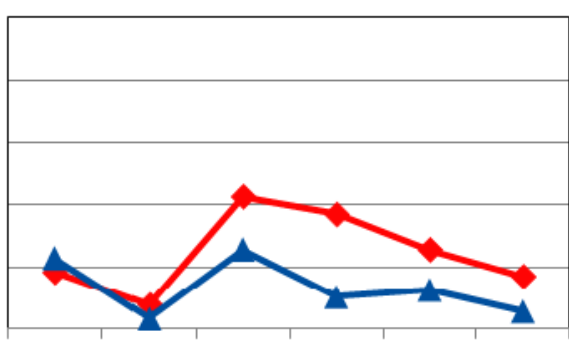

200320042005200620072008

Line Operators

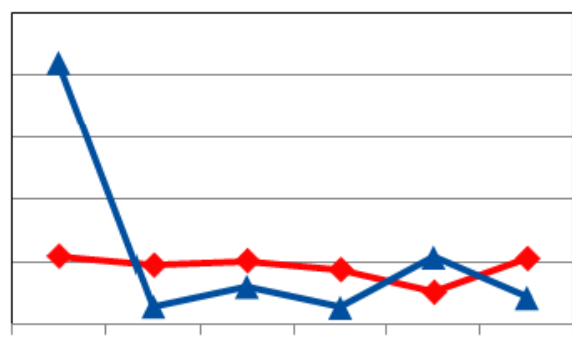

200320042005200620072008

Year

*Standardized to age distribution of 2000 U.S. population. 


\section{Sentinel Health Events for Occupations (SHEOs)}

An occupational sentinel health event (SHEO) is a disease, disability, or death that is likely to be occupationally related. Although sentinel health events may indicate an occupational exposure, many may result from nonoccupational exposures. Sentinel health events are therefore assessed in two categories:

Definite Sentinel Health Events: Diseases that are unlikely to occur in the absence of an occupational exposure (e.g., asbestosis).

Possible Sentinel Health Events: Diseases that may be occupational but can also occur in the absence of an occupational exposure (e.g., lung cancer or carpal tunnel syndrome).

Figure 16. Characteristics of SHEOs by Gender

\begin{tabular}{|l|c|c|c|c|}
\hline \multirow{2}{*}{} & \multicolumn{2}{|c|}{$\begin{array}{c}\text { Total Number of } \\
\text { SHEO Diagnoses }\end{array}$} & \multicolumn{2}{c|}{$\begin{array}{c}\text { Total Number of } \\
\text { Days Absent }\end{array}$} \\
\cline { 2 - 5 } & Men & Women & Men & W omen \\
\hline Definite & 2 & 0 & 124 & 0 \\
\hline Possible & 2 & 2 & 27 & 6 \\
\hline Total & 4 & 2 & 151 & 6 \\
\hline
\end{tabular}

Figure 17. SHEO Diagnoses by Gender

\begin{tabular}{|l|c|c|}
\hline \multirow{2}{*}{ Diagnoses } & \multicolumn{2}{c|}{ Gender } \\
\cline { 2 - 3 } & Women & M en \\
\hline Carpal Tunnel Syndrome & 2 & 1 \\
\hline Other Conditions & 0 & 3 \\
\hline
\end{tabular}


Occupational Safety and Health Administration (OSHA)-Recordable Events

Figure 18. OSHA-Recordable Events by Gender and Age

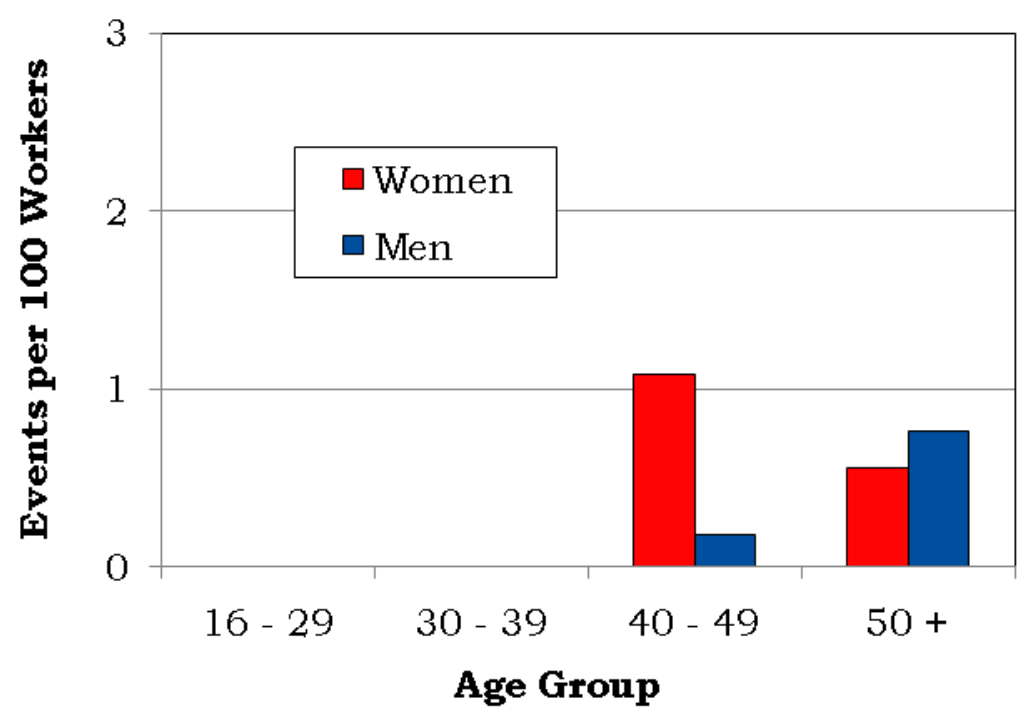

Figure 19. OSHA-Recordable Events by Job Category and Gender

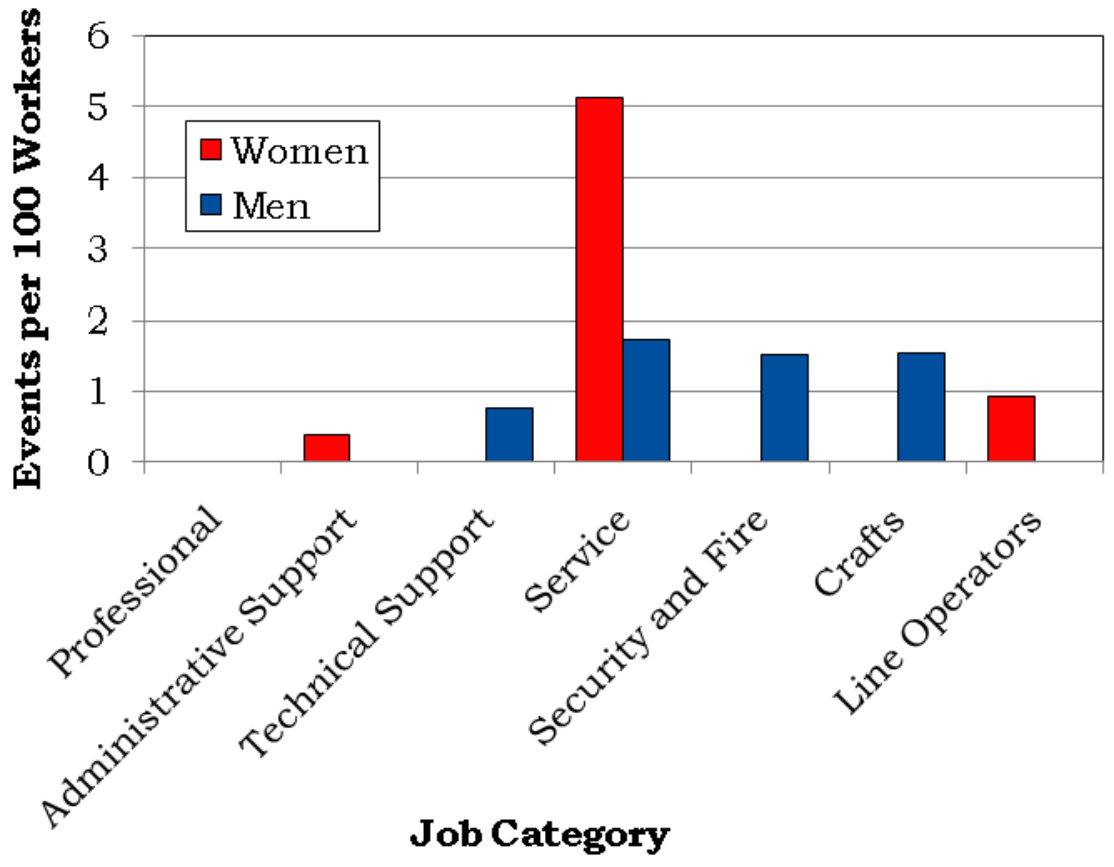




\section{Diagnostic and Accident Categories for OSHA-Recordable Events}

Figure 20. OSHA-Recordable Diagnoses by Diagnostic Category and Gender

\begin{tabular}{|l|c|c|}
\hline \multirow{2}{*}{ Diagnostic Category } & \multicolumn{2}{c|}{ Gender } \\
\cline { 2 - 3 } & Women & M en \\
\hline Cancer & $\mathbf{0}$ & $\mathbf{1}$ \\
\hline Digestive & $\mathbf{0}$ & $\mathbf{1}$ \\
\hline Musculoskeletal & $\mathbf{5}$ & $\mathbf{9}$ \\
\hline Nervous System & $\mathbf{3}$ & $\mathbf{0}$ \\
\hline Respiratory & $\mathbf{1}$ & $\mathbf{0}$ \\
\hline Unspecified Symptoms & $\mathbf{0}$ & $\mathbf{2}$ \\
\hline Injury & $\mathbf{2}$ & $\mathbf{5}$ \\
\hline Other Sprains \& Strains & $\mathbf{0}$ & $\mathbf{1}$ \\
\hline Open Wounds - Upper Limb & $\mathbf{1}$ & $\mathbf{1}$ \\
\hline Burns & $\mathbf{0}$ & $\mathbf{1}$ \\
\hline Unspecified Injuries & $\mathbf{1}$ & $\mathbf{2}$ \\
\hline
\end{tabular}

Figure 21. OSHA-Recordable Accidents by Type and Gender

\begin{tabular}{|l|c|c|}
\hline \multirow{2}{*}{ Accident Category } & \multicolumn{2}{c|}{ Gender } \\
\cline { 2 - 3 } & Women & Men \\
\cline { 2 - 3 } & $\begin{array}{c}\text { Number of } \\
\text { Accidents }\end{array}$ & $\begin{array}{c}\text { Number of } \\
\text { Accidents }\end{array}$ \\
\hline Falls & $\mathbf{0}$ & $\mathbf{2}$ \\
\hline Other Accidents & $\mathbf{3}$ & $\mathbf{6}$ \\
\hline Cutting/Piercing Instrument/Object & $\mathbf{1}$ & $\mathbf{0}$ \\
\hline Hot, Corrosive, or Caustic Material/Steam & $\mathbf{0}$ & $\mathbf{1}$ \\
\hline Overexertion/Strenuous Movements & $\mathbf{1}$ & $\mathbf{5}$ \\
\hline Repetitive Trauma & $\mathbf{1}$ & $\mathbf{0}$ \\
\hline Total & $\mathbf{3}$ & $\mathbf{8}$ \\
\hline
\end{tabular}




\section{Rates of OSHA-Recordable Events}

Figure 22. OSHA-Recordable Rates by Age and Job Categories Among Women, All Diagnoses Combined
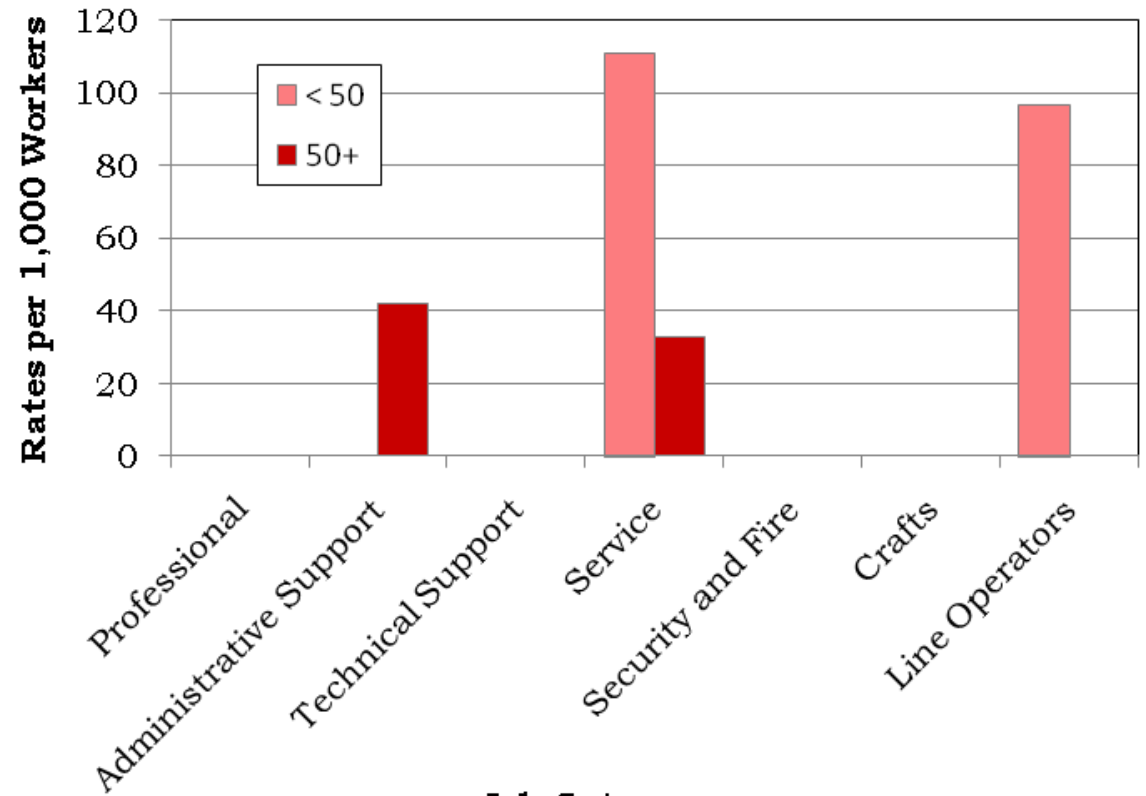

Job Category

Figure 23. OSHA-Recordable Rates by Age and Job Categories Among Men, All Diagnoses Combined

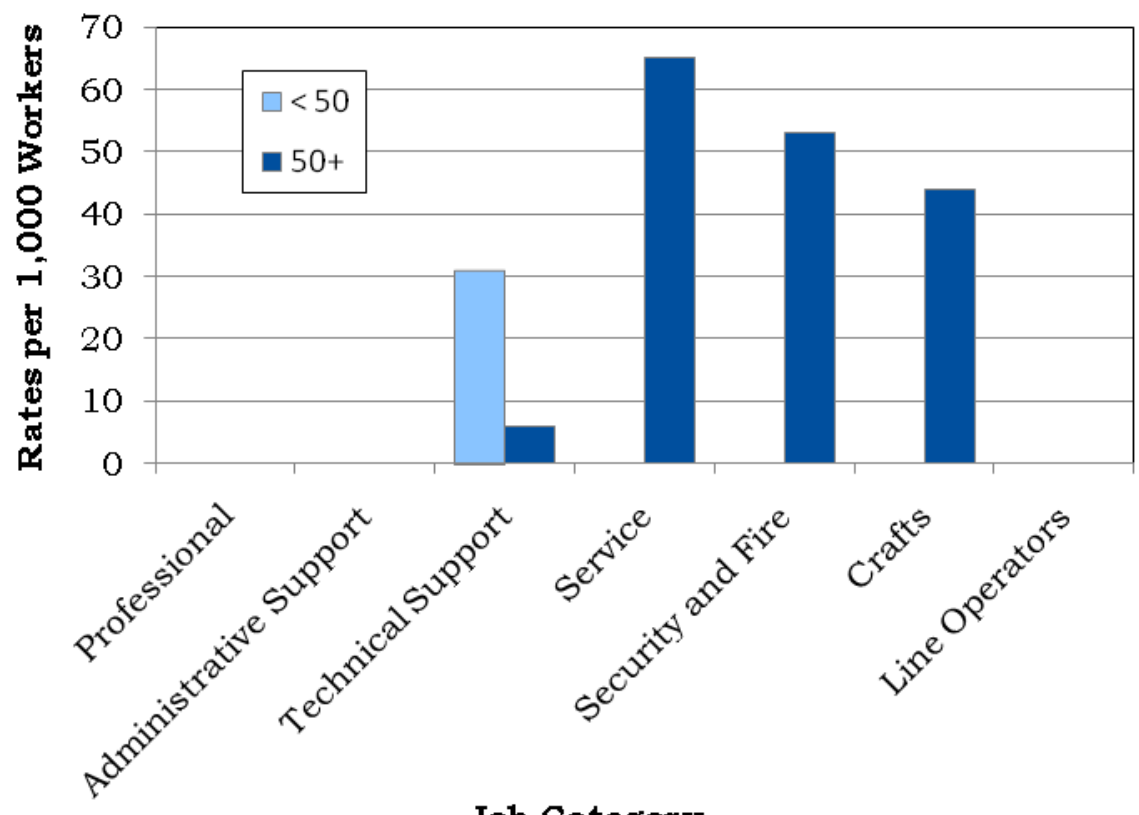

Job Category 


\section{Time Trends for OSHA-Recordable Events}

Figure 24. Age-Adjusted Rates for All OSHA-Recordable Diagnoses Combined Among Women and Men by Job Category from 2003 to 2008*

Professional

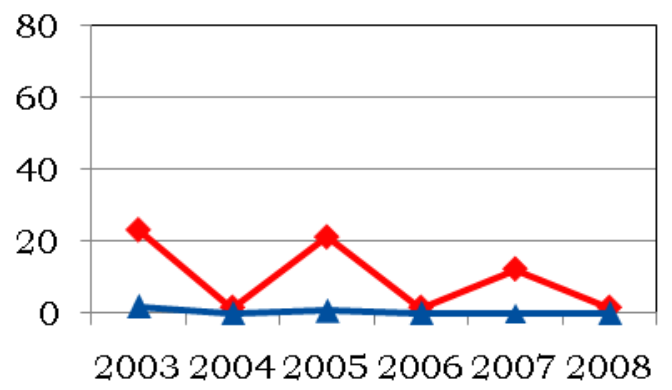

Administrative Sup port
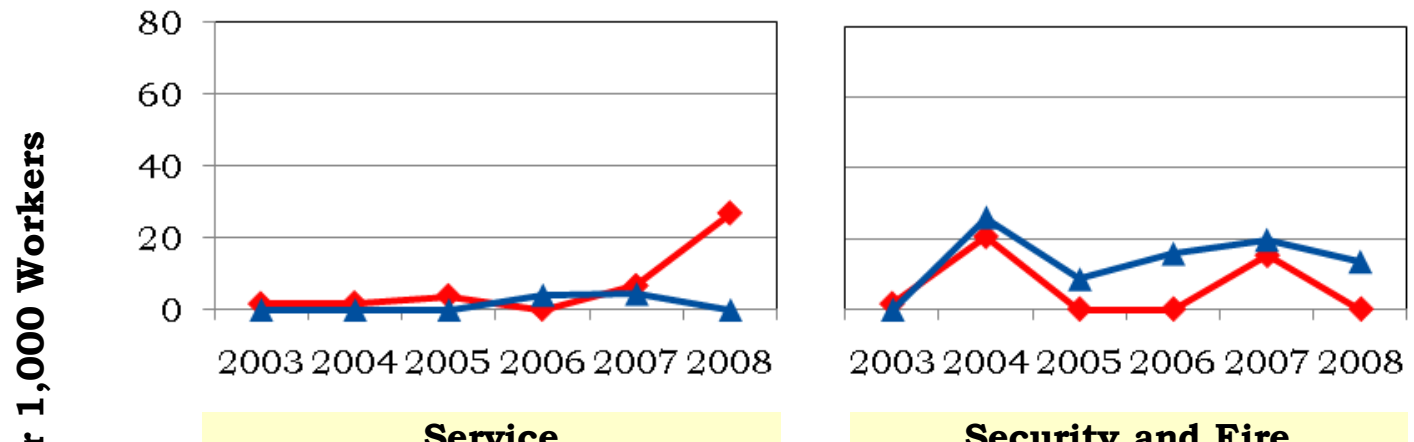

200320042005200620072008

Service

Security and Fire

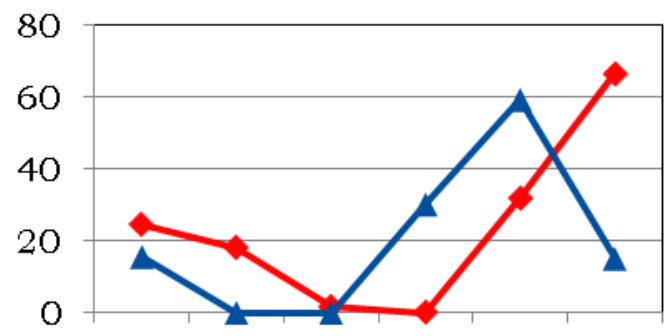

200320042005200620072008

Crafts

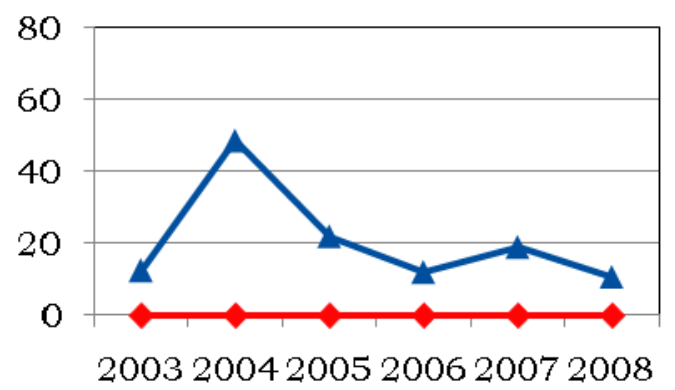

Technical Support

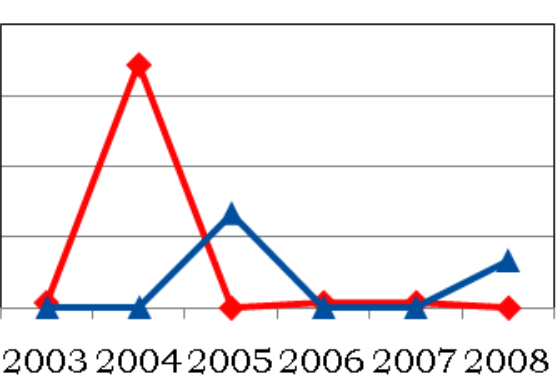

Line Operators

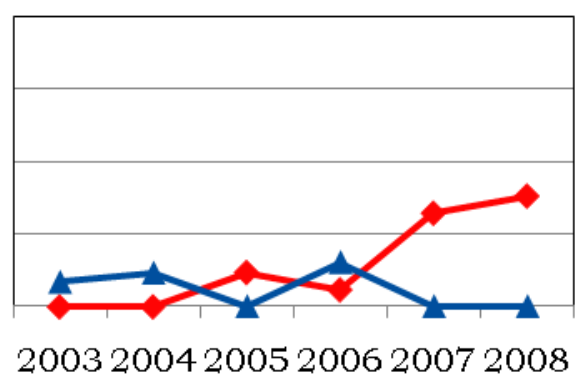

Year

*Standardized to age distribution of 2000 U.S. population. 
Appendices 
Kansas City Plant 2008

Absence Data

Appendix A. Work Force by Gender, Age, and Job Category

\begin{tabular}{|c|c|c|c|c|c|c|c|c|c|c|c|}
\hline \multirow{3}{*}{ Job Category } & \multicolumn{5}{|c|}{ Women } & \multicolumn{5}{|c|}{ Men } & \multirow[b]{3}{*}{ TOTAI } \\
\hline & \multicolumn{4}{|c|}{ Age Group } & \multirow[b]{2}{*}{ TOTAL } & \multicolumn{4}{|c|}{ Age Group } & \multirow[b]{2}{*}{ TOTAL } & \\
\hline & $16-29$ & $30-39$ & $40-49$ & $50+$ & & $16-29$ & $30-39$ & $40-49$ & $\mathbf{5 0}+$ & & \\
\hline Professional & 35 & 21 & 49 & 44 & 149 & 87 & 94 & 277 & 401 & 859 & 1,008 \\
\hline Administrative Support & 12 & 25 & 87 & 142 & 266 & 24 & 29 & 77 & 130 & 260 & 526 \\
\hline Technical Support & 1 & 2 & 14 & 43 & 60 & 18 & 23 & 57 & 167 & 265 & 325 \\
\hline Service & 4 & 1 & 4 & 30 & 39 & 16 & 2 & 9 & 31 & 58 & 97 \\
\hline Security and Fire & 1 & 1 & 5 & 5 & 12 & 4 & 9 & 15 & 38 & 66 & 78 \\
\hline Crafts & 0 & 0 & 3 & 15 & 18 & 5 & 22 & 70 & 228 & 325 & 343 \\
\hline Line Operators & 5 & 3 & 23 & 77 & 108 & 37 & 20 & 46 & 61 & 164 & 272 \\
\hline TOTAL & 58 & 53 & 185 & 356 & 652 & 191 & 199 & 551 & 1,056 & 1,997 & 2,649 \\
\hline
\end{tabular}

Appendix B. Age Distribution of the Work Force by Gender

\begin{tabular}{|l|r|r|r|r|r|r|r|r|}
\hline \multirow{3}{*}{ Year } & \multicolumn{4}{|c|}{ Women } & \multicolumn{4}{c|}{ Men } \\
\cline { 2 - 9 } & \multicolumn{2}{|c|}{ Percent Distribution by Age Group } & \multicolumn{3}{|c|}{ Percent Distribution by Age Group } \\
\cline { 2 - 9 } & $\mathbf{1 6}-\mathbf{2 9}$ & $\mathbf{3 0}-\mathbf{3 9}$ & $\mathbf{4 0}-\mathbf{4 9}$ & $\mathbf{5 0}+$ & $\mathbf{1 6}-\mathbf{2 9}$ & $\mathbf{3 0}-\mathbf{3 9}$ & $\mathbf{4 0}-\mathbf{4 9}$ & $\mathbf{5 0}+$ \\
\hline $\mathbf{2 0 0 2}$ & 9.74 & 9.62 & 40.68 & 39.95 & 8.47 & 8.60 & 42.40 & 40.53 \\
\hline $\mathbf{2 0 0 3}$ & 6.88 & 9.04 & 40.76 & 43.31 & 8.02 & 7.46 & 42.54 & 41.98 \\
\hline $\mathbf{2 0 0 4}$ & 10.52 & 7.79 & 37.98 & 43.72 & 9.04 & 7.25 & 40.96 & 42.75 \\
\hline $\mathbf{2 0 0 5}$ & 9.86 & 7.46 & 35.63 & 47.04 & 8.51 & 7.66 & 37.94 & 45.88 \\
\hline $\mathbf{2 0 0 6}$ & 9.23 & 6.85 & 33.78 & 50.15 & 8.31 & 7.81 & 34.78 & 49.10 \\
\hline $\mathbf{2 0 0 7}$ & 9.57 & 7.10 & 30.71 & 52.62 & 9.43 & 8.31 & 31.12 & 51.14 \\
\hline $\mathbf{2 0 0 8}$ & 8.90 & 8.13 & 28.37 & 54.60 & 9.56 & 9.96 & 27.59 & 52.88 \\
\hline
\end{tabular}


Kansas City Plant 2008

Absence Data

Appendix C. Total Number of Workers Who Reported at Least One Absence by Gender, Age, and Job Category*

\begin{tabular}{|c|c|c|c|c|c|c|c|c|c|c|c|}
\hline \multirow{3}{*}{ Job Category } & \multicolumn{5}{|c|}{ Women } & \multicolumn{5}{|c|}{ Men } & \multirow[b]{3}{*}{ TOTAL } \\
\hline & \multicolumn{4}{|c|}{ Age Group } & \multirow[b]{2}{*}{ TOTAL } & \multicolumn{4}{|c|}{ Age Group } & \multirow[b]{2}{*}{ TOTAL } & \\
\hline & $16-29$ & 30 - 39 & $40-49$ & $50+$ & & $16-29$ & 30 - 39 & $40-49$ & $50+$ & & \\
\hline Professional & 2 & 1 & 1 & 5 & 9 & 2 & 3 & 13 & 23 & 41 & 50 \\
\hline Administrative Support & 0 & 0 & 11 & 13 & 24 & 0 & 2 & 6 & 8 & 16 & 40 \\
\hline Technical Support & 0 & 0 & 3 & 2 & 5 & 2 & 0 & 5 & 11 & 18 & 23 \\
\hline Service & 1 & 1 & 1 & 5 & 8 & 2 & 0 & 1 & 5 & 8 & 16 \\
\hline Security and Fire & 0 & 0 & 0 & 2 & 2 & 0 & 0 & 2 & 4 & 6 & 8 \\
\hline Crafts & 0 & 0 & 0 & 2 & 2 & 0 & 0 & 4 & 24 & 28 & 30 \\
\hline Line Operators & 0 & 1 & 2 & 6 & 9 & 1 & 0 & 3 & 7 & 11 & 20 \\
\hline TOTAL & 3 & 3 & 18 & 35 & 59 & 7 & 5 & 34 & 82 & 128 & 187 \\
\hline
\end{tabular}

*Only those job categories and gender/age combinations with at least one absence appear in this table.

Appendix D. Total Number of Absences by Gender, Age, and Job Category*

\begin{tabular}{|c|c|c|c|c|c|c|c|c|c|c|c|}
\hline \multirow{3}{*}{ Job Category } & \multicolumn{5}{|c|}{ Women } & \multicolumn{5}{|c|}{ Men } & \multirow[b]{3}{*}{ TOTAL } \\
\hline & \multicolumn{4}{|c|}{ Age Group } & \multirow[b]{2}{*}{ TOTAL } & \multicolumn{4}{|c|}{ Age Group } & \multirow[b]{2}{*}{ TOTAL } & \\
\hline & $16-29$ & 30 - 39 & $40-49$ & $50+$ & & $16-29$ & 30 - 39 & $40-49$ & $50+$ & & \\
\hline Professional & 3 & 1 & 1 & 6 & 11 & 2 & 3 & 14 & 30 & 49 & 60 \\
\hline Administrative Support & 0 & 0 & 12 & 16 & 28 & 0 & 2 & 6 & 9 & 17 & 45 \\
\hline Technical Support & 0 & 0 & 4 & 2 & 6 & 2 & 0 & 5 & 12 & 19 & 25 \\
\hline Service & 1 & 1 & 2 & 6 & 10 & 2 & 0 & 1 & 5 & 8 & 18 \\
\hline Security and Fire & 0 & 0 & 0 & 2 & 2 & 0 & 0 & 2 & 4 & 6 & 8 \\
\hline Crafts & 0 & 0 & 0 & 2 & 2 & 0 & 0 & 5 & 26 & 31 & 33 \\
\hline Line Operators & 0 & 1 & 2 & 6 & 9 & 1 & 0 & 3 & 8 & 12 & 21 \\
\hline TOTAL & 4 & 3 & 21 & 40 & 68 & 7 & 5 & 36 & 94 & 142 & 210 \\
\hline
\end{tabular}

*Only those job categories and gender/age combinations with at least one absence appear in this table. 
Kansas City Plant 2008

Absence Data

Appendix E. Distribution of the Number of Calendar Days Missed per Absence by Gender and Age*

\begin{tabular}{|c|c|c|c|c|c|c|c|c|c|c|c|}
\hline \multirow{3}{*}{$\begin{array}{c}\text { Number of } \\
\text { Calendar Days }\end{array}$} & \multicolumn{5}{|c|}{ Women } & \multicolumn{5}{|c|}{ Men } & \multirow[b]{3}{*}{ TOTAL } \\
\hline & \multicolumn{4}{|c|}{ Age Group } & \multirow[b]{2}{*}{ TOTAL } & \multicolumn{4}{|c|}{ Age Group } & \multirow[b]{2}{*}{ TOTAL } & \\
\hline & $16-29$ & $30-39$ & $40-49$ & $50+$ & & $16-29$ & 30 - 39 & $40-49$ & $50+$ & & \\
\hline$<15$ & 1 & 3 & 9 & 19 & 32 & 6 & 3 & 22 & 43 & 74 & 106 \\
\hline $15-28$ & 3 & 0 & 2 & 6 & 11 & 0 & 1 & 4 & 26 & 31 & 42 \\
\hline $29-42$ & 0 & 0 & 2 & 6 & 8 & 1 & 0 & 2 & 9 & 12 & 20 \\
\hline $43-56$ & 0 & 0 & 4 & 4 & 8 & 0 & 0 & 3 & 8 & 11 & 19 \\
\hline $57-91$ & 0 & 0 & 3 & 3 & 6 & 0 & 1 & 2 & 3 & 6 & 12 \\
\hline $92-182$ & 0 & 0 & 1 & 1 & 2 & 0 & 0 & 3 & 5 & 8 & 10 \\
\hline $183+$ & 0 & 0 & 0 & 1 & 1 & 0 & 0 & 0 & 0 & 0 & 1 \\
\hline TOTAL & 4 & 3 & 21 & 40 & 68 & 7 & 5 & 36 & 94 & 142 & 210 \\
\hline
\end{tabular}

*Only those gender/age combinations with at least one absence appear in this table. 
Kansas City Plant 2008

Absence Data

Appendix F. Distribution of the Number of Calendar Days Missed per Absence by Gender and Job Category*

Women

\begin{tabular}{|c|c|c|c|c|c|c|c|c|}
\hline \multirow{2}{*}{$\begin{array}{c}\text { Number of } \\
\text { Calendar Days }\end{array}$} & \multicolumn{7}{|c|}{ Job Category } & \multirow[b]{2}{*}{ TOTAL } \\
\hline & Professional & $\begin{array}{c}\text { Administrative } \\
\text { Support }\end{array}$ & Technical Support & Service & Security and Fire & Crafts & Line Operators & \\
\hline$<15$ & 5 & 16 & 0 & 7 & 1 & 1 & 2 & 32 \\
\hline $15-28$ & 5 & 2 & 0 & 1 & 1 & 0 & 2 & 11 \\
\hline $57-91$ & 0 & 4 & 1 & 0 & 0 & 0 & 1 & 6 \\
\hline $92-182$ & 0 & 0 & 2 & 0 & 0 & 0 & 0 & 2 \\
\hline $183+$ & 0 & 0 & 0 & 0 & 0 & 0 & 1 & 1 \\
\hline TOTAL & 11 & 28 & 6 & 10 & 2 & 2 & 9 & 68 \\
\hline
\end{tabular}

Men

\begin{tabular}{|c|c|c|c|c|c|c|c|c|}
\hline \multirow{2}{*}{$\begin{array}{l}\text { Number of } \\
\text { Calendar Days }\end{array}$} & \multicolumn{7}{|c|}{ Job Category } & \multirow[b]{2}{*}{ TOTAL } \\
\hline & Professional & $\begin{array}{l}\text { Administrative } \\
\text { Support }\end{array}$ & Technical Support & Service & Security and Fire & Crafts & Line Operators & \\
\hline$<15$ & 31 & 10 & 8 & 2 & 1 & 15 & 7 & 74 \\
\hline $15-28$ & 12 & 1 & 4 & 2 & 3 & 7 & 2 & 31 \\
\hline $57-91$ & 3 & 0 & 0 & 1 & 0 & 2 & 0 & 6 \\
\hline $92-182$ & 1 & 1 & 2 & 1 & 0 & 2 & 1 & 8 \\
\hline $183+$ & 0 & 0 & 0 & 0 & 0 & 0 & 0 & 0 \\
\hline TOTAL & 49 & 17 & 19 & 8 & 6 & 31 & 12 & 142 \\
\hline
\end{tabular}

*Only those gender/job category combinations with at least one absence appear in this table. 


\section{Kansas City Plant 2008}

Absence Data

Appendix G. Number of Diagnoses in Each Diagnostic Category by Gender and Age*

\begin{tabular}{|c|c|c|c|c|c|c|}
\hline & & \multicolumn{5}{|c|}{ Women } \\
\hline & & \multicolumn{4}{|c|}{ Age Group } & \multirow[b]{2}{*}{ TOTAL } \\
\hline & & $16-29$ & $30-39$ & $40-49$ & $50+$ & \\
\hline Diagnostic Category & ICD-9-CM Code & & & & & \\
\hline INFECTIOUS \& PARASITIC DISEASES (DIS) & 001-139 & 0 & 0 & 0 & 4 & 4 \\
\hline -Intestinal Infectious Dis & 001-009 & 0 & 0 & 0 & 0 & 0 \\
\hline -Other Bacterial Dis & 030-041 & 0 & 0 & 0 & 1 & 1 \\
\hline -Viral Dis with Exanthem & 050-057 & 0 & 0 & 0 & 1 & 1 \\
\hline -Other Viral Dis \& Chlamydiae & 070-079 & 0 & 0 & 0 & 1 & 1 \\
\hline -Mycoses & $110-118$ & 0 & 0 & 0 & 1 & 1 \\
\hline MALIGNANT NEOPLASMS & $140-208,230-234$ & 0 & 0 & 1 & 8 & 9 \\
\hline -Digestive \& Peritoneal & $150-159$ & 0 & 0 & 1 & 0 & 1 \\
\hline -Respiratory \& Intrathoracic & $160-165$ & 0 & 0 & 0 & 0 & 0 \\
\hline -Breast & 174-175 & 0 & 0 & 0 & 1 & 1 \\
\hline -Genitourinary & 179-189 & 0 & 0 & 0 & 1 & 1 \\
\hline -Other \& Unspecified Sites & 190, 193-199 & 0 & 0 & 0 & 6 & 6 \\
\hline -Nervous System & 191-192 & 0 & 0 & 0 & 0 & 0 \\
\hline BENIGN \& UNCERTAIN NEOPLASMS & 210-229, 235-239 & 1 & 0 & 2 & 0 & 3 \\
\hline ENDOCRINE/METABOLIC/IMMUNITY & 240-279 & 0 & 0 & 1 & 2 & 3 \\
\hline -Thyroid Gland Disorders & $240-246$ & 0 & 0 & 1 & 0 & 1 \\
\hline -Other Endocrine Gland Dis & $250-259$ & 0 & 0 & 0 & 1 & 1 \\
\hline -Other Metabolic \& Immunity Disorders & 270-279 & 0 & 0 & 0 & 1 & 1 \\
\hline BLOOD \& BLOOD-FORMING ORGANS & 280-289 & 0 & 0 & 1 & 0 & 1 \\
\hline MENTAL DISORDERS & $290-319$ & 0 & 0 & 0 & 1 & 1 \\
\hline -Psychoses & 290-299 & 0 & 0 & 0 & 0 & 0 \\
\hline -Non-Psychotic Disorders & $300-302,306-316$ & 0 & 0 & 0 & 1 & 1 \\
\hline NERVOUS SYSTEM (NS) \& SENSE ORGANS & $320-389$ & 0 & 0 & 3 & 4 & 7 \\
\hline -Inflammatory Dis of Central NS & $320-326$ & 0 & 0 & 0 & 0 & 0 \\
\hline -Hereditary/Degenerative Central NS Dis & 330-337 & 0 & 0 & 0 & 1 & 1 \\
\hline -Other Disorders of Central NS & $340-349$ & 0 & 0 & 2 & 0 & 2 \\
\hline -Disorders of Peripheral NS & $350-359$ & 0 & 0 & 0 & 2 & 2 \\
\hline -Disorders of Eye & $360-379$ & 0 & 0 & 1 & 1 & 2 \\
\hline CIRCULATORY SYSTEM & $390-459$ & 0 & 0 & 2 & 3 & 5 \\
\hline -Hypertensive Dis & $401-405$ & 0 & 0 & 0 & 0 & 0 \\
\hline -Ischemic Heart Dis & $410-414$ & 0 & 0 & 0 & 1 & 1 \\
\hline -Dis of Pulmonary Circulation & $415-417$ & 0 & 0 & 1 & 0 & 1 \\
\hline -Other Heart Dis & $420-429$ & 0 & 0 & 1 & 1 & 2 \\
\hline -Cerebrovascular Dis & $430-438$ & 0 & 0 & 0 & 0 & 0 \\
\hline -Dis of Arteries \& Capillaries & $440-448$ & 0 & 0 & 0 & 1 & 1 \\
\hline -Dis of Veins, Lymphatics, Other & 451-459 & 0 & 0 & 0 & 0 & 0 \\
\hline RESPIRATORY SYSTEM & $460-519$ & 0 & 1 & 4 & 8 & 13 \\
\hline
\end{tabular}

*Only those diagnostic categories and gender/age combinations with at least one occurrence appear in this table. 


\section{Kansas City Plant 2008}

\section{Absence Data}

Appendix G. Number of Diagnoses in Each Diagnostic Category by Gender and Age*

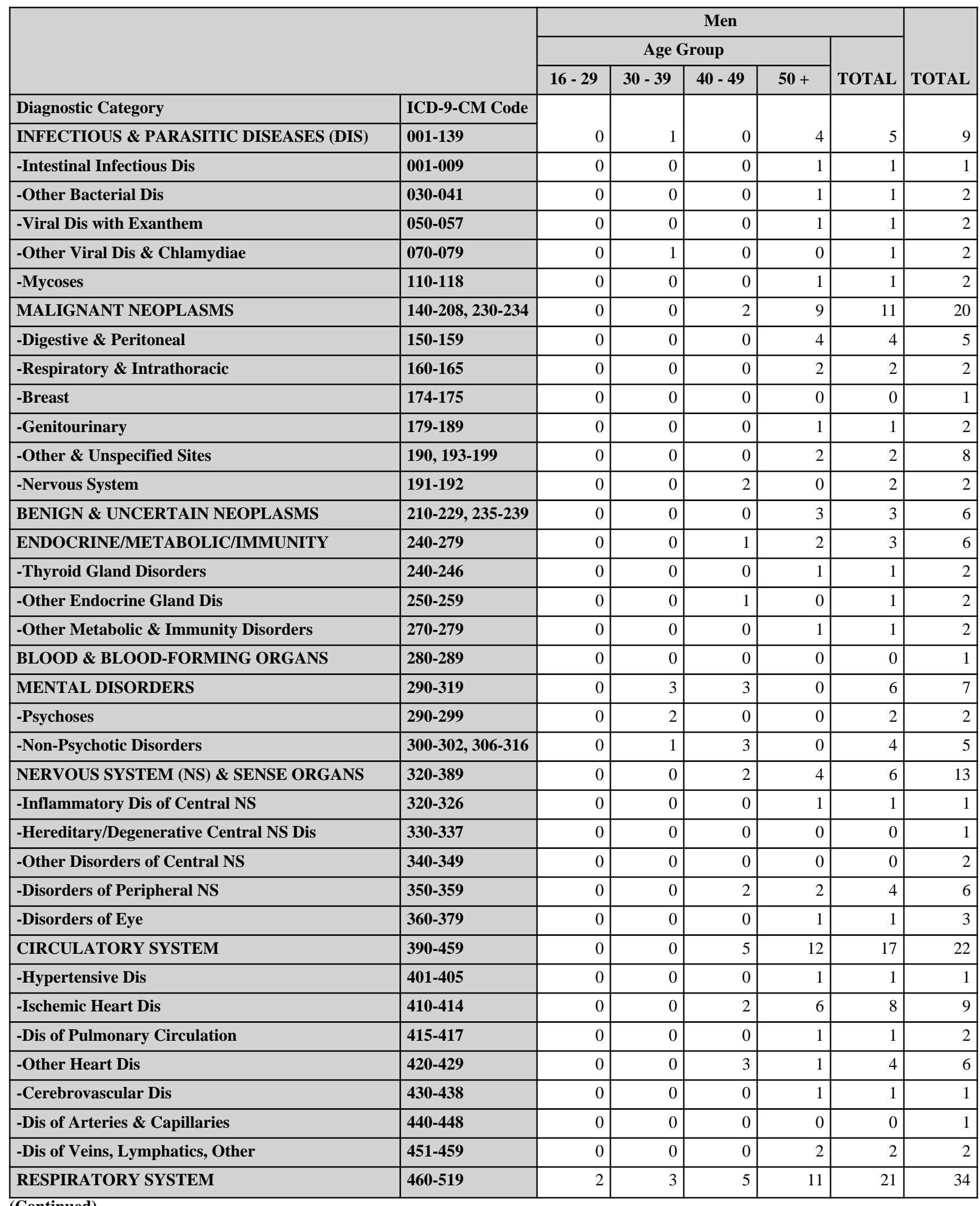

*Only those diagnostic categories and gender/age combinations with at least one occurrence appear in this table. 


\section{Kansas City Plant 2008}

Absence Data

Appendix G. Number of Diagnoses in Each Diagnostic Category by Gender and Age*

\begin{tabular}{|c|c|c|c|c|c|c|}
\hline & & \multicolumn{5}{|c|}{ Women } \\
\hline & & \multicolumn{4}{|c|}{ Age Group } & \multirow[b]{2}{*}{ TOTAL } \\
\hline & & $16-29$ & $30-39$ & $40-49$ & $\mathbf{5 0 +}$ & \\
\hline Diagnostic Category & ICD-9-CM Code & \multirow[b]{2}{*}{0} & \multirow[b]{2}{*}{0} & \multirow[b]{2}{*}{3} & \multirow[b]{2}{*}{2} & \multirow[b]{2}{*}{5} \\
\hline -Acute Respiratory Infections & $460-466$ & & & & & \\
\hline -Other Dis Upper Respiratory Tract & $470-478$ & 0 & 1 & 0 & 1 & 2 \\
\hline -Pneumonia \& Influenza & $480-487$ & 0 & 0 & 1 & 3 & 4 \\
\hline -Chronic Obstructive Dis & $490-496$ & 0 & 0 & 0 & 0 & 0 \\
\hline -Lung Dis from External Agents & $500-508$ & 0 & 0 & 0 & 1 & 1 \\
\hline -Other Respiratory Dis & $510-519$ & 0 & 0 & 0 & 1 & 1 \\
\hline DIGESTIVE SYSTEM & $520-579$ & 0 & 0 & 2 & 5 & 7 \\
\hline -Esophagus, Stomach, Duodenum & $530-537$ & 0 & 0 & 0 & 0 & 0 \\
\hline -Appendicitis & $540-543$ & 0 & 0 & 0 & 1 & 1 \\
\hline -Hernia & $550-553$ & 0 & 0 & 0 & 2 & 2 \\
\hline -Enteritis, Colitis & $555-558$ & 0 & 0 & 0 & 0 & 0 \\
\hline -Other Intestinal Dis & $560-569$ & 0 & 0 & 0 & 0 & 0 \\
\hline -Other Digestive Dis & $570-579$ & 0 & 0 & 2 & 2 & 4 \\
\hline GENITOURINARY SYSTEM & $580-629$ & 0 & 0 & 6 & 3 & 9 \\
\hline -Other Urinary Dis & $590-599$ & 0 & 0 & 1 & 1 & 2 \\
\hline -Male Genital Organ Dis & $600-608$ & 0 & 0 & 0 & 0 & 0 \\
\hline -Breast Disorders & $610-611$ & 0 & 0 & 2 & 0 & 2 \\
\hline -Other Female Disorders & $617-629$ & 0 & 0 & 3 & 2 & 5 \\
\hline SKIN \& SUBCUTANEOUS TISSUE & $680-709$ & 0 & 1 & 0 & 1 & 2 \\
\hline -Infections & $680-686$ & 0 & 1 & 0 & 1 & 2 \\
\hline $\begin{array}{l}\text { MUSCULOSKELETAL \& CONNECTIVE } \\
\text { TISSUE }\end{array}$ & 710-739 & 2 & 2 & 6 & 13 & 23 \\
\hline -Arthropathies & $710-719$ & 0 & 0 & 2 & 6 & 8 \\
\hline -Dorsopathies & $720-724$ & 0 & 1 & 2 & 2 & 5 \\
\hline -Rheumatism, Excluding Back & 725-729 & 2 & 1 & 2 & 2 & 7 \\
\hline -Other Dis \& Acquired Deformities & 730-739 & 0 & 0 & 0 & 3 & 3 \\
\hline CONGENITAL ANOMALIES & 740-759 & 0 & 0 & 0 & 0 & 0 \\
\hline $\begin{array}{l}\text { SYMPTOMS, SIGNS, \& ILL-DEFINED } \\
\text { CONDITIONS }\end{array}$ & 780-799 & 1 & 1 & 3 & 10 & 15 \\
\hline -Symptoms & 780-789 & 1 & 1 & 2 & 9 & 13 \\
\hline -Non-Specific Abnormal Findings & \begin{tabular}{|l|}
$790-796$ \\
\end{tabular} & 0 & 0 & 1 & 1 & 2 \\
\hline INJURY \& POISONING & $800-999$ & 0 & 0 & 3 & 7 & 10 \\
\hline -Fracture - Skull & $800-804$ & 0 & 0 & 0 & 1 & 1 \\
\hline -Fracture - Neck, Trunk & 805-809 & 0 & 0 & 0 & 0 & 0 \\
\hline -Fracture - Upper Limb & $810-819$ & 0 & 0 & 0 & 0 & 0 \\
\hline -Fracture - Lower Limb & 820-829 & 0 & 0 & 0 & 0 & 0 \\
\hline -Dislocation & $830-839$ & 0 & 0 & 0 & 3 & 3 \\
\hline
\end{tabular}

(Continued)

*Only those diagnostic categories and gender/age combinations with at least one occurrence appear in this table. 


\section{Kansas City Plant 2008}

Absence Data

Appendix G. Number of Diagnoses in Each Diagnostic Category by Gender and Age*

\begin{tabular}{|c|c|c|c|c|c|c|c|}
\hline & & \multicolumn{5}{|c|}{ Men } & \multirow[b]{3}{*}{ TOTAL } \\
\hline & & \multicolumn{4}{|c|}{ Age Group } & \multirow[b]{2}{*}{ TOTAL } & \\
\hline & & $16-29$ & $30-39$ & $40-49$ & $50+$ & & \\
\hline -Acute Respiratory Infections & $460-466$ & 0 & 0 & 1 & 0 & 1 & 6 \\
\hline -Other Dis Upper Respiratory Tract & $470-478$ & 2 & 2 & 1 & 2 & 7 & 9 \\
\hline -Chronic Obstructive Dis & $490-496$ & 0 & 0 & 0 & 1 & 1 & 1 \\
\hline -Lung Dis from External Agents & $500-508$ & 0 & 0 & 0 & 0 & 0 & 1 \\
\hline -Other Respiratory Dis & $510-519$ & 0 & 0 & 0 & 0 & 0 & 1 \\
\hline DIGESTIVE SYSTEM & $520-579$ & 1 & 0 & 10 & 18 & 29 & 36 \\
\hline -Enteritis, Colitis & $555-558$ & 1 & 0 & 0 & 0 & 1 & 1 \\
\hline -Other Intestinal Dis & $560-569$ & 0 & 0 & 1 & 4 & 5 & 5 \\
\hline -Other Digestive Dis & $570-579$ & 0 & 0 & 3 & 6 & 9 & 13 \\
\hline GENITOURINARY SYSTEM & $580-629$ & 1 & 0 & 0 & 3 & 4 & 13 \\
\hline -Other Urinary Dis & $590-599$ & 1 & 0 & 0 & 2 & 3 & 5 \\
\hline -Male Genital Organ Dis & $600-608$ & 0 & 0 & 0 & 1 & 1 & 1 \\
\hline -Breast Disorders & $610-611$ & 0 & 0 & 0 & 0 & 0 & 2 \\
\hline -Other Female Disorders & $617-629$ & 0 & 0 & 0 & 0 & 0 & 5 \\
\hline -Dorsopathies & $720-724$ & 0 & 0 & 1 & 11 & 12 & 17 \\
\hline -Rheumatism, Excluding Back & $725-729$ & 0 & 0 & 1 & 11 & 12 & 19 \\
\hline -Other Dis \& Acquired Deformities & $730-739$ & 0 & 0 & 0 & 0 & 0 & 3 \\
\hline CONGENITAL ANOMALIES & 740-759 & 0 & 0 & 1 & 0 & 1 & 1 \\
\hline $\begin{array}{l}\text { SYMPTOMS, SIGNS, \& ILL-DEFINED } \\
\text { CONDITIONS }\end{array}$ & 780-799 & 3 & 3 & 7 & 22 & 35 & 50 \\
\hline -Symptoms & 780-789 & 3 & 3 & 7 & 21 & 34 & 47 \\
\hline -Non-Specific Abnormal Findings & $790-796$ & 0 & 0 & 0 & 1 & 1 & 3 \\
\hline INJURY \& POISONING & $800-999$ & 3 & 1 & 18 & 27 & 49 & 59 \\
\hline -Fracture - Skull & $800-804$ & 0 & 0 & 0 & 0 & 0 & 1 \\
\hline -Fracture - Neck, Trunk & 805-809 & 0 & 0 & 3 & 4 & 7 & 7 \\
\hline -Fracture - Upper Limb & 810-819 & 0 & 0 & 2 & 0 & 2 & 2 \\
\hline -Fracture - Lower Limb & $820-829$ & 0 & 0 & 4 & 0 & 4 & 4 \\
\hline -Dislocation & $830-839$ & 0 & 0 & 1 & 11 & 12 & 15 \\
\hline
\end{tabular}

(Continued)

*Only those diagnostic categories and gender/age combinations with at least one occurrence appear in this table. 


\section{Kansas City Plant 2008}

Absence Data

Appendix G. Number of Diagnoses in Each Diagnostic Category by Gender and Age*

\begin{tabular}{|c|c|c|c|c|c|c|}
\hline & & \multicolumn{5}{|c|}{ Women } \\
\hline & & \multicolumn{4}{|c|}{ Age Group } & \multirow[b]{2}{*}{ TOTAL } \\
\hline & & $16-29$ & $30-39$ & $40-49$ & $50+$ & \\
\hline Diagnostic Category & ICD-9-CM Code & \multirow[b]{2}{*}{0} & \multirow[b]{2}{*}{0} & \multirow[b]{2}{*}{0} & \multirow[b]{2}{*}{0} & \multirow[b]{2}{*}{0} \\
\hline -Sprains \& Strains - Other & $840-845,848$ & & & & & \\
\hline -Intracranial Injury & $850-854$ & 0 & 0 & 0 & 1 & 1 \\
\hline -Open Wound - Head, Neck, Trunk & $870-879$ & 0 & 0 & 0 & 1 & 1 \\
\hline -Open Wound - Upper Limb & $880-887$ & 0 & 0 & 0 & 0 & 0 \\
\hline -Open Wound - Lower Limb & $890-897$ & 0 & 0 & 1 & 0 & 1 \\
\hline -Late Effects of Accident & $905-909$ & 0 & 0 & 0 & 0 & 0 \\
\hline -Superficial Injury & $910-919$ & 0 & 0 & 0 & 0 & 0 \\
\hline -Contusion & $920-924$ & 0 & 0 & 0 & 0 & 0 \\
\hline -Foreign Body Entering Orifice & 930-939 & 0 & 0 & 1 & 0 & 1 \\
\hline -Burns & 940-949 & 0 & 0 & 0 & 0 & 0 \\
\hline -Complications \& Unspecified Injuries & 958-959 & 0 & 0 & 0 & 1 & 1 \\
\hline -Toxic Effects - Non-medicinal & 980-989 & 0 & 0 & 0 & 0 & 0 \\
\hline -Complications of Surgical/Medical Care & 996-999 & 0 & 0 & 1 & 0 & 1 \\
\hline $\begin{array}{l}\text { HEALTH STATUS/HEALTH SERVICE } \\
\text { CONTACT }\end{array}$ & V01-V82 & 0 & 0 & 0 & 2 & 2 \\
\hline -Personal \& Family History & V10-V19 & 0 & 0 & 0 & 0 & 0 \\
\hline -Health Services Reproduction/Development & V20-V29 & 0 & 0 & 0 & 0 & 0 \\
\hline -Specific Procedures/Aftercare & V50-V59 & 0 & 0 & 0 & 1 & 1 \\
\hline -Examination \& Investigation & V70-V82 & 0 & 0 & 0 & 1 & 1 \\
\hline
\end{tabular}

*Only those diagnostic categories and gender/age combinations with at least one occurrence appear in this table. 


\section{Kansas City Plant 2008}

Absence Data

Appendix G. Number of Diagnoses in Each Diagnostic Category by Gender and Age*

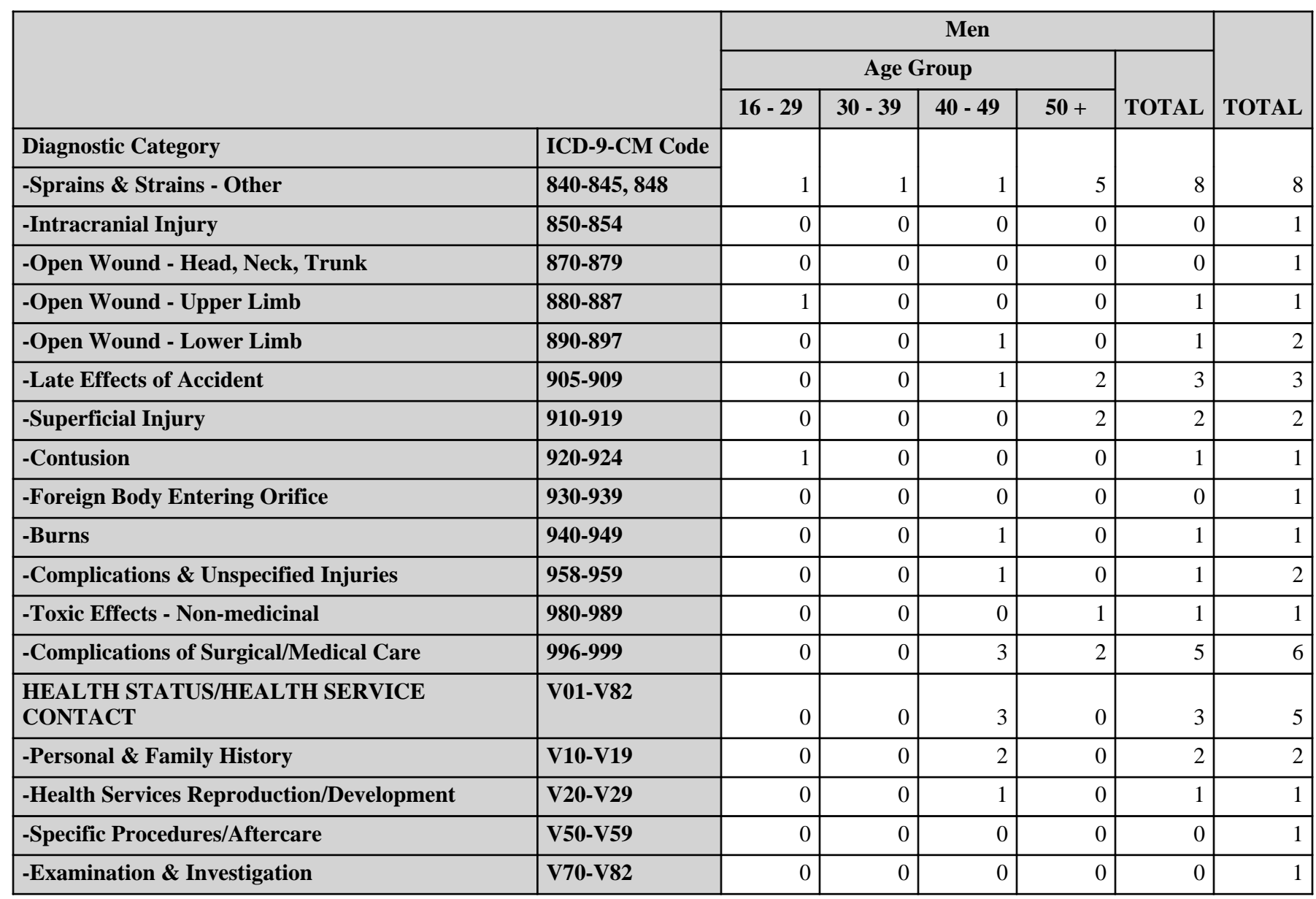

\begin{tabular}{|c|c|c|c|c|c|c|c|c|c|c|c|}
\hline & \multicolumn{5}{|c|}{ Women } & \multicolumn{5}{|c|}{ Men } & \multirow[b]{3}{*}{ TOTAL } \\
\hline & \multicolumn{4}{|c|}{ Age Group } & \multirow[b]{2}{*}{ TOTAL } & \multicolumn{4}{|c|}{ Age Group } & \multirow[b]{2}{*}{ TOTAL } & \\
\hline & $16-29$ & 30 - 39 & $40-49$ & $50+$ & & $16-29$ & 30 - 39 & 40 - 49 & $50+$ & & \\
\hline Diagnostic Category & \multirow[b]{2}{*}{4} & \multirow[b]{2}{*}{5} & \multirow[b]{2}{*}{34} & \multirow[b]{2}{*}{71} & \multirow[b]{2}{*}{114} & \multirow[b]{2}{*}{11} & \multirow[b]{2}{*}{11} & \multirow[b]{2}{*}{65} & \multirow[b]{2}{*}{149} & \multirow[b]{2}{*}{236} & \multirow[b]{2}{*}{350} \\
\hline Total & & & & & & & & & & & \\
\hline
\end{tabular}

*Only those diagnostic categories and gender/age combinations with at least one occurrence appear in this table. 


\section{Kansas City Plant 2008}

Absence Data

Appendix H. Total Number of Calendar Days Absent in Each Diagnostic Category by Gender and Age*

\begin{tabular}{|c|c|c|c|c|c|c|}
\hline & & \multicolumn{5}{|c|}{ Women } \\
\hline & & \multicolumn{4}{|c|}{ Age Group } & \multirow[b]{2}{*}{ TOTAL } \\
\hline & & $16-29$ & $30-39$ & $40-49$ & $\mathbf{5 0 +}$ & \\
\hline Diagnostic Category & ICD-9-CM Code & \multirow[b]{2}{*}{0} & \multirow[b]{2}{*}{0} & \multirow[b]{2}{*}{0} & \multirow[b]{2}{*}{59} & \multirow[b]{2}{*}{59} \\
\hline INFECTIOUS \& PARASITIC DISEASES (DIS) & 001-139 & & & & & \\
\hline MALIGNANT NEOPLASMS & 140-208, 230-234 & 0 & 0 & 61 & 319 & 380 \\
\hline BENIGN \& UNCERTAIN NEOPLASMS & 210-229, 235-239 & 16 & 0 & 86 & 0 & 102 \\
\hline ENDOCRINE/METABOLIC/IMMUNITY & $240-279$ & 0 & 0 & 11 & 88 & 99 \\
\hline BLOOD \& BLOOD-FORMING ORGANS & $280-289$ & 0 & 0 & 43 & 0 & 43 \\
\hline MENTAL DISORDERS & 290-319 & 0 & 0 & 0 & 77 & 77 \\
\hline NERVOUS SYSTEM (NS) \& SENSE ORGANS & 320-389 & 0 & 0 & 197 & 13 & 210 \\
\hline CIRCULATORY SYSTEM & $390-459$ & 0 & 0 & 72 & 90 & 162 \\
\hline RESPIRATORY SYSTEM & $460-519$ & 0 & 10 & 32 & 79 & 121 \\
\hline DIGESTIVE SYSTEM & $520-579$ & 0 & 0 & 90 & 106 & 196 \\
\hline GENITOURINARY SYSTEM & $580-629$ & 0 & 0 & 191 & 53 & 244 \\
\hline SKIN \& SUBCUTANEOUS TISSUE & 680-709 & 0 & 5 & 0 & 58 & 63 \\
\hline $\begin{array}{l}\text { MUSCULOSKELETAL \& CONNECTIVE } \\
\text { TISSUE }\end{array}$ & $710-739$ & 42 & 14 & 334 & 449 & 839 \\
\hline CONGENITAL ANOMALIES & $740-759$ & 0 & 0 & 0 & 0 & 0 \\
\hline $\begin{array}{l}\text { SYMPTOMS, SIGNS, \& ILL-DEFINED } \\
\text { CONDITIONS }\end{array}$ & 780-799 & 7 & 10 & 98 & 163 & 278 \\
\hline INJURY \& POISONING & $800-999$ & 0 & 0 & 210 & 250 & 460 \\
\hline
\end{tabular}

\footnotetext{
*Absences with >1 ICD-9-CM code in the same diagnostic category were counted only once. Only those diagnostic categories and gender/age combinations with at least one occurrence appear in this table.
} 


\section{Kansas City Plant 2008}

Absence Data

Appendix H. Total Number of Calendar Days Absent in Each Diagnostic Category by Gender and Age*

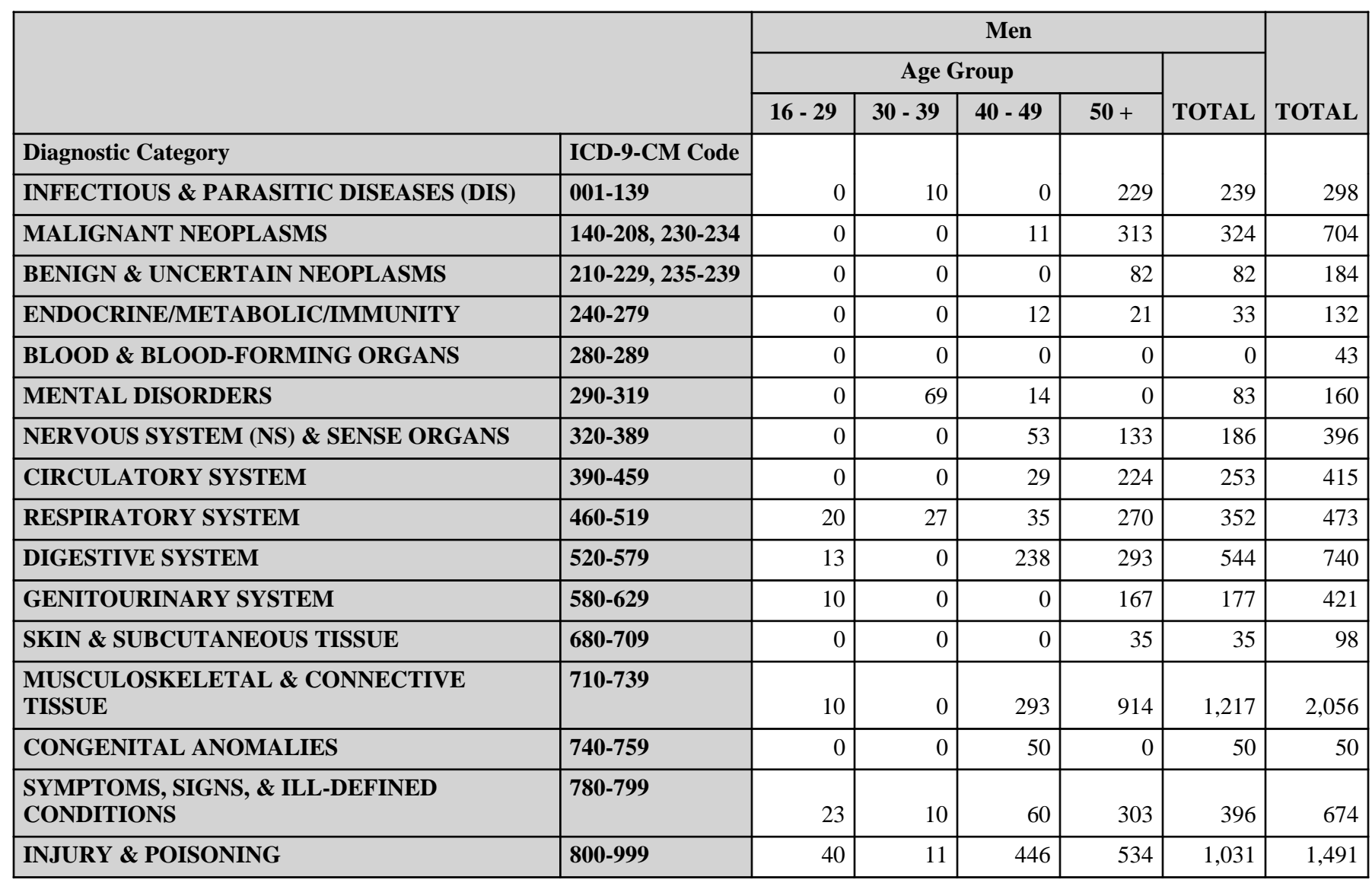

\footnotetext{
*Absences with >1 ICD-9-CM code in the same diagnostic category were counted only once. Only those diagnostic categories and gender/age combinations with at least one occurrence appear in this table.
} 


\section{Kansas City Plant 2008}

Absence Data

Appendix I. Number of Diagnoses in Each Diagnostic Category by Gender and Job Category*

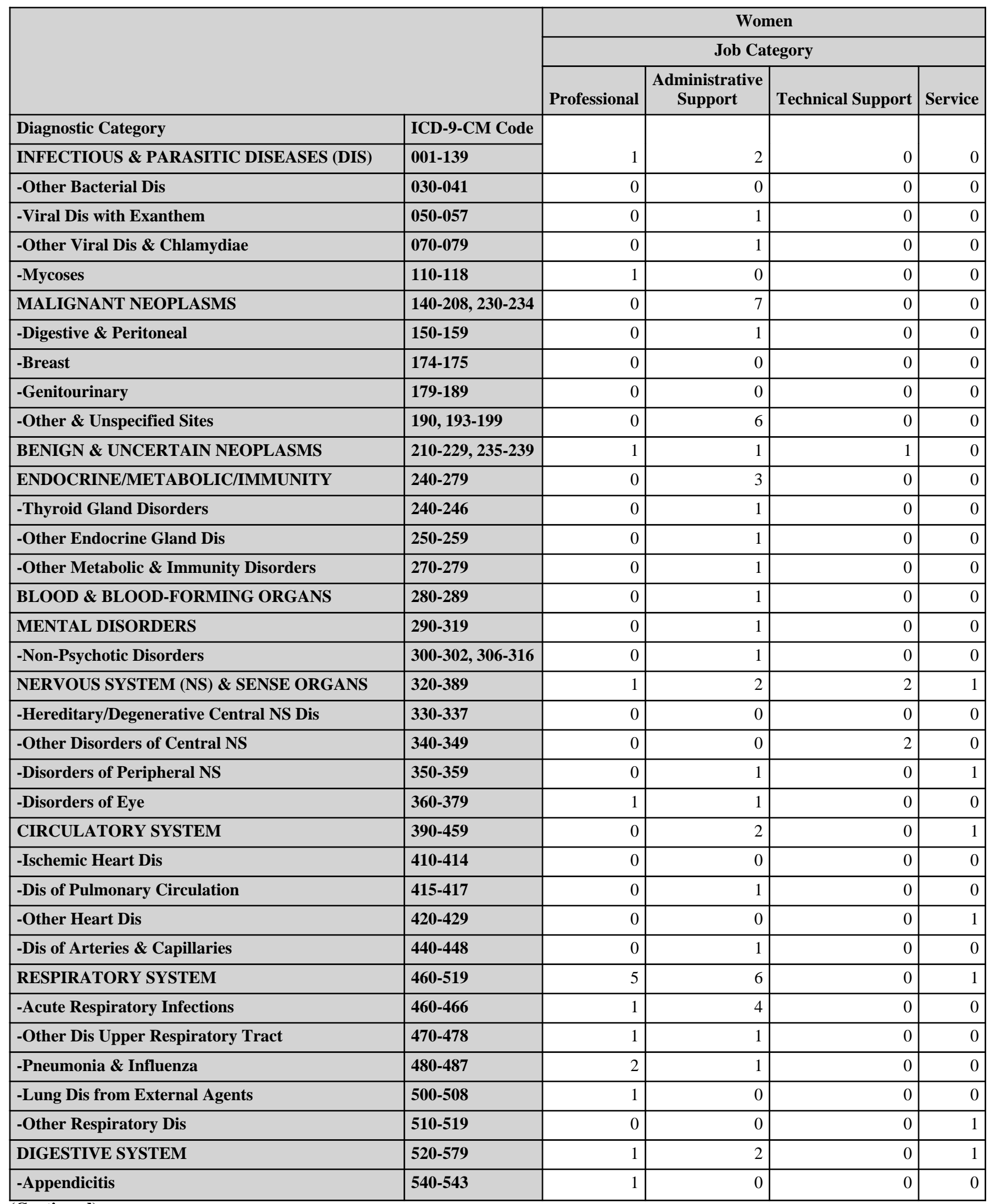

(Continued)

*Only those diagnostic categories and gender/job category combinations with at least one occurrence appear in this table. 


\section{Kansas City Plant 2008}

Absence Data

Appendix I. Number of Diagnoses in Each Diagnostic Category by Gender and Job Category*

\begin{tabular}{|c|c|c|c|c|c|}
\hline & & \multicolumn{4}{|c|}{ Women } \\
\hline & & \multicolumn{3}{|c|}{ Job Category } & \multirow[b]{2}{*}{ TOTAL } \\
\hline & & Security and Fire & Crafts & Line Operators & \\
\hline Diagnostic Category & ICD-9-CM Code & \multirow[b]{2}{*}{1} & \multirow[b]{2}{*}{0} & \multirow[b]{2}{*}{0} & \multirow[b]{2}{*}{4} \\
\hline INFECTIOUS \& PARASITIC DISEASES (DIS) & 001-139 & & & & \\
\hline -Other Bacterial Dis & $030-041$ & 1 & 0 & 0 & 1 \\
\hline -Viral Dis with Exanthem & 050-057 & 0 & 0 & 0 & 1 \\
\hline -Other Viral Dis \& Chlamydiae & 070-079 & 0 & 0 & 0 & 1 \\
\hline -Mycoses & $110-118$ & 0 & 0 & 0 & 1 \\
\hline MALIGNANT NEOPLASMS & 140-208, 230-234 & 0 & 0 & 2 & 9 \\
\hline -Digestive \& Peritoneal & $150-159$ & 0 & 0 & 0 & 1 \\
\hline -Breast & 174-175 & 0 & 0 & 1 & 1 \\
\hline -Genitourinary & 179-189 & 0 & 0 & 1 & 1 \\
\hline -Other \& Unspecified Sites & 190, 193-199 & 0 & 0 & 0 & 6 \\
\hline BENIGN \& UNCERTAIN NEOPLASMS & 210-229, 235-239 & 0 & 0 & 0 & 3 \\
\hline ENDOCRINE/METABOLIC/IMMUNITY & $240-279$ & 0 & 0 & 0 & 3 \\
\hline -Thyroid Gland Disorders & $240-246$ & 0 & 0 & 0 & 1 \\
\hline -Other Endocrine Gland Dis & $250-259$ & 0 & 0 & 0 & 1 \\
\hline -Other Metabolic \& Immunity Disorders & $270-279$ & 0 & 0 & 0 & 1 \\
\hline BLOOD \& BLOOD-FORMING ORGANS & $280-289$ & 0 & 0 & 0 & 1 \\
\hline MENTAL DISORDERS & $290-319$ & 0 & 0 & 0 & 1 \\
\hline -Non-Psychotic Disorders & $300-302,306-316$ & 0 & 0 & 0 & 1 \\
\hline NERVOUS SYSTEM (NS) \& SENSE ORGANS & $320-389$ & 1 & 0 & 0 & 7 \\
\hline -Hereditary/Degenerative Central NS Dis & $330-337$ & 1 & 0 & 0 & 1 \\
\hline -Other Disorders of Central NS & $340-349$ & 0 & 0 & 0 & 2 \\
\hline -Disorders of Peripheral NS & $350-359$ & 0 & 0 & 0 & 2 \\
\hline -Disorders of Eye & $360-379$ & 0 & 0 & 0 & 2 \\
\hline CIRCULATORY SYSTEM & $390-459$ & 0 & 0 & 2 & 5 \\
\hline -Ischemic Heart Dis & $410-414$ & 0 & 0 & 1 & 1 \\
\hline -Dis of Pulmonary Circulation & $415-417$ & 0 & 0 & 0 & 1 \\
\hline -Other Heart Dis & $420-429$ & 0 & 0 & 1 & 2 \\
\hline -Dis of Arteries \& Capillaries & $440-448$ & 0 & 0 & 0 & 1 \\
\hline RESPIRATORY SYSTEM & $460-519$ & 0 & 1 & 0 & 13 \\
\hline -Acute Respiratory Infections & $460-466$ & 0 & 0 & 0 & 5 \\
\hline -Other Dis Upper Respiratory Tract & $470-478$ & 0 & 0 & 0 & 2 \\
\hline -Pneumonia \& Influenza & $480-487$ & 0 & 1 & 0 & 4 \\
\hline -Lung Dis from External Agents & $500-508$ & 0 & 0 & 0 & 1 \\
\hline -Other Respiratory Dis & $510-519$ & 0 & 0 & 0 & 1 \\
\hline DIGESTIVE SYSTEM & $520-579$ & 0 & 1 & 2 & 7 \\
\hline -Appendicitis & $540-543$ & 0 & 0 & 0 & 1 \\
\hline
\end{tabular}

(Continued)

*Only those diagnostic categories and gender/job category combinations with at least one occurrence appear in this table. 


\section{Kansas City Plant 2008}

Absence Data

Appendix I. Number of Diagnoses in Each Diagnostic Category by Gender and Job Category*

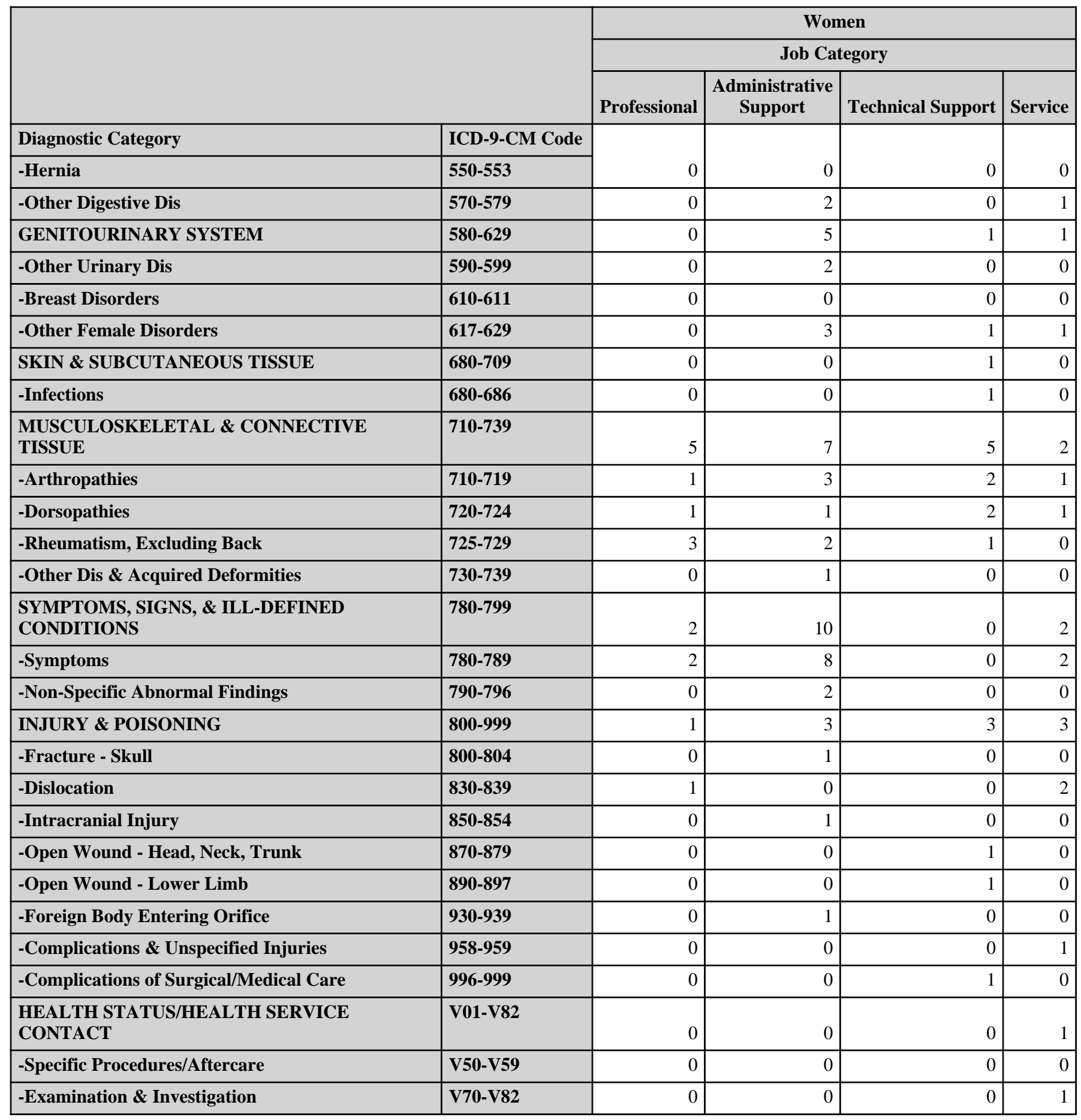

*Only those diagnostic categories and gender/job category combinations with at least one occurrence appear in this table. 


\section{Kansas City Plant 2008}

Absence Data

Appendix I. Number of Diagnoses in Each Diagnostic Category by Gender and Job Category*

\begin{tabular}{|c|c|c|c|c|c|}
\hline & & \multicolumn{4}{|c|}{ Women } \\
\hline & & \multicolumn{3}{|c|}{ Job Category } & \multirow[b]{2}{*}{ TOTAL } \\
\hline & & Security and Fire & Crafts & Line Operators & \\
\hline Diagnostic Category & ICD-9-CM Code & \multirow[b]{2}{*}{0} & \multirow[b]{2}{*}{1} & \multirow[b]{2}{*}{1} & \multirow[b]{2}{*}{2} \\
\hline -Hernia & $550-553$ & & & & \\
\hline -Other Digestive Dis & $570-579$ & 0 & 0 & 1 & 4 \\
\hline GENITOURINARY SYSTEM & $580-629$ & 0 & 0 & 2 & 9 \\
\hline -Other Urinary Dis & $590-599$ & 0 & 0 & 0 & 2 \\
\hline -Breast Disorders & $610-611$ & 0 & 0 & 2 & 2 \\
\hline -Other Female Disorders & $617-629$ & 0 & 0 & 0 & 5 \\
\hline SKIN \& SUBCUTANEOUS TISSUE & $680-709$ & 0 & 0 & 1 & 2 \\
\hline -Infections & $680-686$ & 0 & 0 & 1 & 2 \\
\hline $\begin{array}{l}\text { MUSCULOSKELETAL \& CONNECTIVE } \\
\text { TISSUE }\end{array}$ & $710-739$ & 2 & 0 & 2 & 23 \\
\hline -Arthropathies & $710-719$ & 1 & 0 & 0 & 8 \\
\hline -Dorsopathies & $720-724$ & 0 & 0 & 0 & 5 \\
\hline -Rheumatism, Excluding Back & $725-729$ & 0 & 0 & 1 & 7 \\
\hline -Other Dis \& Acquired Deformities & $730-739$ & 1 & 0 & 1 & 3 \\
\hline $\begin{array}{l}\text { SYMPTOMS, SIGNS, \& ILL-DEFINED } \\
\text { CONDITIONS }\end{array}$ & $780-799$ & 0 & 0 & 1 & 15 \\
\hline -Symptoms & 780-789 & 0 & 0 & 1 & 13 \\
\hline -Non-Specific Abnormal Findings & $790-796$ & 0 & 0 & 0 & 2 \\
\hline INJURY \& POISONING & 800-999 & 0 & 0 & 0 & 10 \\
\hline -Fracture - Skull & $800-804$ & 0 & 0 & 0 & 1 \\
\hline -Dislocation & 830-839 & 0 & 0 & 0 & 3 \\
\hline -Intracranial Injury & $850-854$ & 0 & 0 & 0 & 1 \\
\hline -Open Wound - Head, Neck, Trunk & 870-879 & 0 & 0 & 0 & 1 \\
\hline -Open Wound - Lower Limb & $890-897$ & 0 & 0 & 0 & 1 \\
\hline -Foreign Body Entering Orifice & 930-939 & 0 & 0 & 0 & 1 \\
\hline -Complications \& Unspecified Injuries & 958-959 & 0 & 0 & 0 & 1 \\
\hline -Complications of Surgical/Medical Care & 996-999 & 0 & 0 & 0 & 1 \\
\hline $\begin{array}{l}\text { HEALTH STATUS/HEALTH SERVICE } \\
\text { CONTACT }\end{array}$ & V01-V82 & 0 & 0 & 1 & 2 \\
\hline -Specific Procedures/Aftercare & V50-V59 & 0 & 0 & 1 & 1 \\
\hline -Examination \& Investigation & V70-V82 & 0 & 0 & 0 & 1 \\
\hline
\end{tabular}

*Only those diagnostic categories and gender/job category combinations with at least one occurrence appear in this table. 
Kansas City Plant 2008

Absence Data

Appendix I. Number of Diagnoses in Each Diagnostic Category by Gender and Job Category*

\begin{tabular}{|c|c|c|c|c|c|c|c|c|}
\hline & \multicolumn{8}{|c|}{ Women } \\
\hline & \multicolumn{7}{|c|}{ Job Category } & \multirow[b]{2}{*}{ TOTAL } \\
\hline & Professional & $\begin{array}{c}\text { Administrative } \\
\text { Support }\end{array}$ & $\begin{array}{c}\text { Technical } \\
\text { Support }\end{array}$ & Service & $\begin{array}{l}\text { Security and } \\
\text { Fire }\end{array}$ & Crafts & $\begin{array}{c}\text { Line } \\
\text { Operators }\end{array}$ & \\
\hline Diagnostic Category & \multirow[b]{2}{*}{17} & \multirow[b]{2}{*}{52} & \multirow[b]{2}{*}{13} & \multirow[b]{2}{*}{13} & \multirow[b]{2}{*}{4} & \multirow[b]{2}{*}{2} & \multirow[b]{2}{*}{13} & \multirow[b]{2}{*}{114} \\
\hline Total & & & & & & & & \\
\hline
\end{tabular}

*Only those diagnostic categories and gender/job category combinations with at least one occurrence appear in this table. 


\section{Kansas City Plant 2008}

Absence Data

Appendix I. Number of Diagnoses in Each Diagnostic Category by Gender and Job Category*

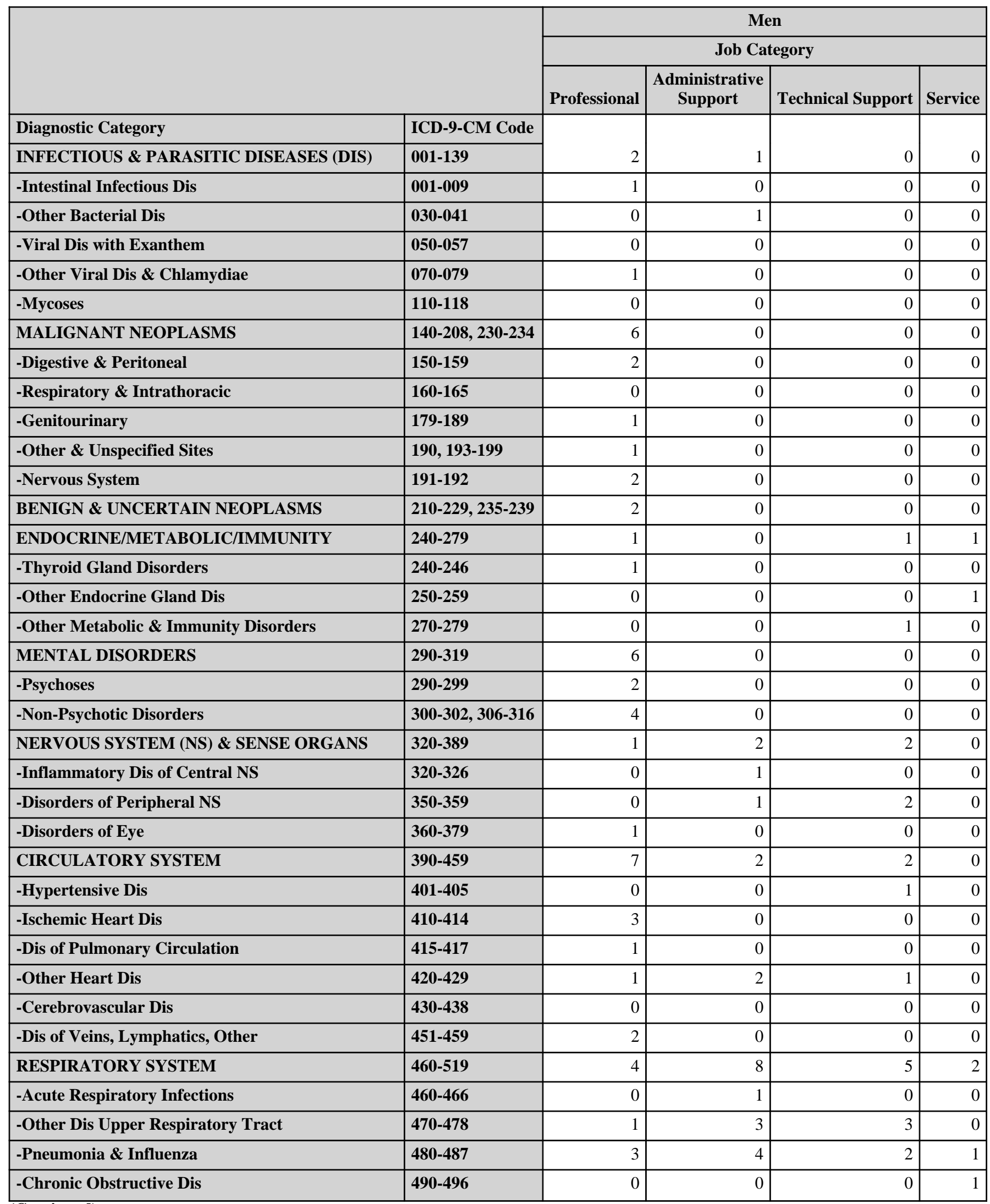

(Continued)

*Only those diagnostic categories and gender/job category combinations with at least one occurrence appear in this table. 


\section{Kansas City Plant 2008}

Absence Data

Appendix I. Number of Diagnoses in Each Diagnostic Category by Gender and Job Category*

\begin{tabular}{|c|c|c|c|c|c|}
\hline & & \multicolumn{4}{|c|}{ Men } \\
\hline & & \multicolumn{3}{|c|}{ Job Category } & \multirow[b]{2}{*}{ TOTAL } \\
\hline & & Security and Fire & Crafts & Line Operators & \\
\hline Diagnostic Category & ICD-9-CM Code & \multirow[b]{2}{*}{0} & \multirow[b]{2}{*}{2} & \multirow[b]{2}{*}{0} & \multirow[b]{2}{*}{5} \\
\hline INFECTIOUS \& PARASITIC DISEASES (DIS) & 001-139 & & & & \\
\hline -Intestinal Infectious Dis & 001-009 & 0 & 0 & 0 & 1 \\
\hline -Other Bacterial Dis & $030-041$ & 0 & 0 & 0 & 1 \\
\hline -Viral Dis with Exanthem & $050-057$ & 0 & 1 & 0 & 1 \\
\hline -Other Viral Dis \& Chlamydiae & 070-079 & 0 & 0 & 0 & 1 \\
\hline -Mycoses & $110-118$ & 0 & 1 & 0 & 1 \\
\hline MALIGNANT NEOPLASMS & 140-208, 230-234 & 0 & 4 & 1 & 11 \\
\hline -Digestive \& Peritoneal & 150-159 & 0 & 1 & 1 & 4 \\
\hline -Respiratory \& Intrathoracic & $160-165$ & 0 & 2 & 0 & 2 \\
\hline -Genitourinary & $179-189$ & 0 & 0 & 0 & 1 \\
\hline -Other \& Unspecified Sites & $190,193-199$ & 0 & 1 & 0 & 2 \\
\hline -Nervous System & 191-192 & 0 & 0 & 0 & 2 \\
\hline BENIGN \& UNCERTAIN NEOPLASMS & 210-229, 235-239 & 0 & 0 & 1 & 3 \\
\hline ENDOCRINE/METABOLIC/IMMUNITY & $240-279$ & 0 & 0 & 0 & 3 \\
\hline -Thyroid Gland Disorders & $240-246$ & 0 & 0 & 0 & 1 \\
\hline -Other Endocrine Gland Dis & $250-259$ & 0 & 0 & 0 & 1 \\
\hline -Other Metabolic \& Immunity Disorders & $270-279$ & 0 & 0 & 0 & 1 \\
\hline MENTAL DISORDERS & 290-319 & 0 & 0 & 0 & 6 \\
\hline -Psychoses & $290-299$ & 0 & 0 & 0 & 2 \\
\hline -Non-Psychotic Disorders & $300-302,306-316$ & 0 & 0 & 0 & 4 \\
\hline NERVOUS SYSTEM (NS) \& SENSE ORGANS & 320-389 & 0 & 1 & 0 & 6 \\
\hline -Inflammatory Dis of Central NS & $320-326$ & 0 & 0 & 0 & 1 \\
\hline -Disorders of Peripheral NS & $350-359$ & 0 & 1 & 0 & 4 \\
\hline -Disorders of Eye & $360-379$ & 0 & 0 & 0 & 1 \\
\hline CIRCULATORY SYSTEM & $390-459$ & 0 & 3 & 3 & 17 \\
\hline -Hypertensive Dis & $401-405$ & 0 & 0 & 0 & 1 \\
\hline -Ischemic Heart Dis & $410-414$ & 0 & 2 & 3 & 8 \\
\hline -Dis of Pulmonary Circulation & $415-417$ & 0 & 0 & 0 & 1 \\
\hline -Other Heart Dis & $420-429$ & 0 & 0 & 0 & 4 \\
\hline -Cerebrovascular Dis & $430-438$ & 0 & 1 & 0 & 1 \\
\hline -Dis of Veins, Lymphatics, Other & 451-459 & 0 & 0 & 0 & 2 \\
\hline RESPIRATORY SYSTEM & $460-519$ & 0 & 1 & 1 & 21 \\
\hline -Acute Respiratory Infections & $460-466$ & 0 & 0 & 0 & 1 \\
\hline -Other Dis Upper Respiratory Tract & $470-478$ & 0 & 0 & 0 & 7 \\
\hline -Pneumonia \& Influenza & $480-487$ & 0 & 1 & 1 & 12 \\
\hline -Chronic Obstructive Dis & $490-496$ & 0 & 0 & 0 & 1 \\
\hline
\end{tabular}

(Continued)

*Only those diagnostic categories and gender/job category combinations with at least one occurrence appear in this table. 


\section{Kansas City Plant 2008}

Absence Data

Appendix I. Number of Diagnoses in Each Diagnostic Category by Gender and Job Category*

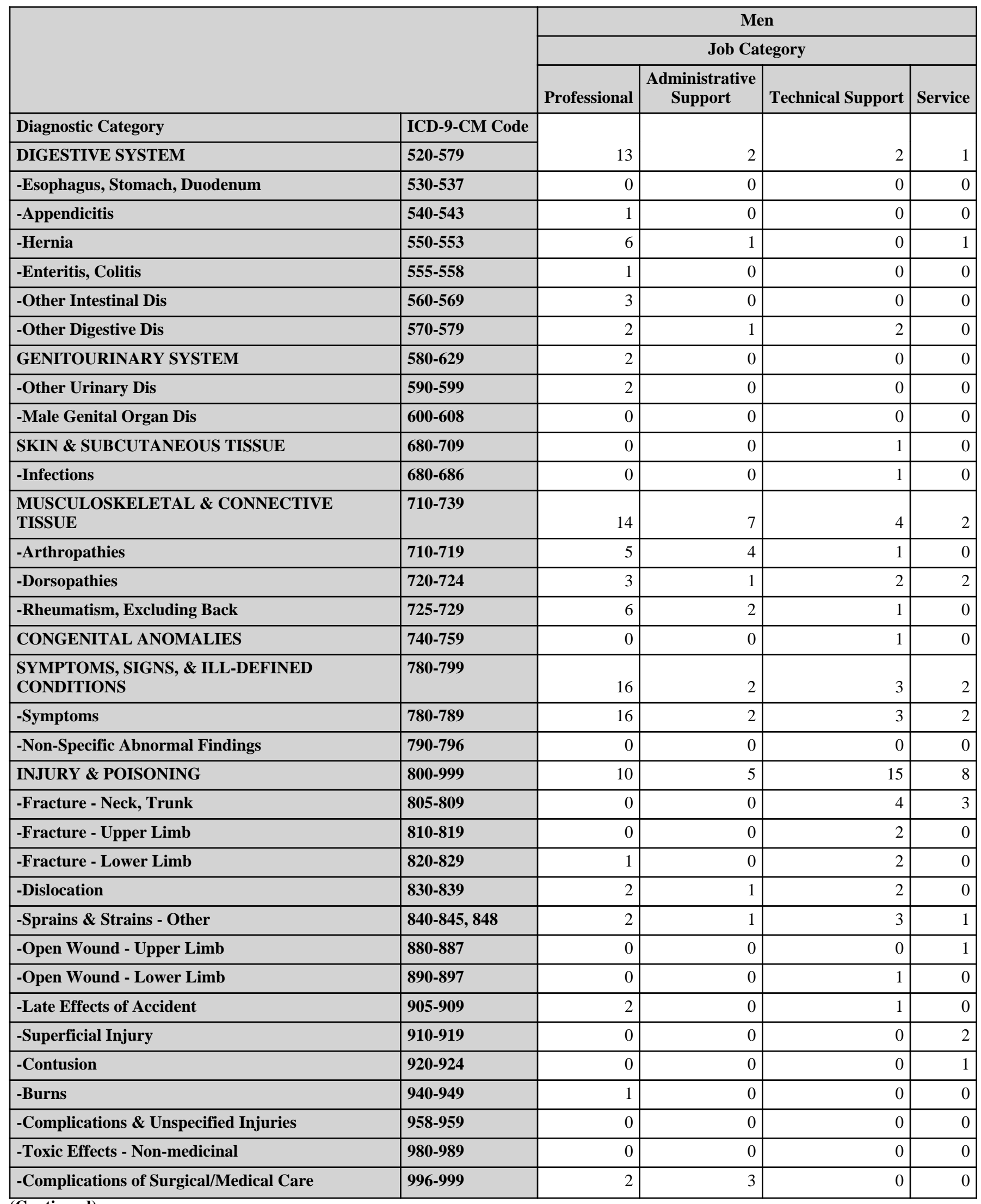

(Continued)

*Only those diagnostic categories and gender/job category combinations with at least one occurrence appear in this table. 


\section{Kansas City Plant 2008}

Absence Data

Appendix I. Number of Diagnoses in Each Diagnostic Category by Gender and Job Category*

\begin{tabular}{|c|c|c|c|c|c|}
\hline & & \multicolumn{4}{|c|}{ Men } \\
\hline & & \multicolumn{3}{|c|}{ Job Category } & \multirow[b]{2}{*}{ TOTAL } \\
\hline & & Security and Fire & Crafts & Line Operators & \\
\hline Diagnostic Category & ICD-9-CM Code & \multirow[b]{2}{*}{1} & \multirow[b]{2}{*}{9} & \multirow[b]{2}{*}{1} & \multirow[b]{2}{*}{29} \\
\hline DIGESTIVE SYSTEM & $520-579$ & & & & \\
\hline -Esophagus, Stomach, Duodenum & $530-537$ & 0 & 1 & 0 & 1 \\
\hline -Appendicitis & $540-543$ & 0 & 0 & 0 & 1 \\
\hline -Hernia & $550-553$ & 1 & 3 & 0 & 12 \\
\hline -Enteritis, Colitis & $555-558$ & 0 & 0 & 0 & 1 \\
\hline -Other Intestinal Dis & $560-569$ & 0 & 1 & 1 & 5 \\
\hline -Other Digestive Dis & $570-579$ & 0 & 4 & 0 & 9 \\
\hline GENITOURINARY SYSTEM & $580-629$ & 0 & 1 & 1 & 4 \\
\hline -Other Urinary Dis & $590-599$ & 0 & 0 & 1 & 3 \\
\hline -Male Genital Organ Dis & $600-608$ & 0 & 1 & 0 & 1 \\
\hline SKIN \& SUBCUTANEOUS TISSUE & $680-709$ & 0 & 0 & 0 & 1 \\
\hline -Infections & $680-686$ & 0 & 0 & 0 & 1 \\
\hline $\begin{array}{l}\text { MUSCULOSKELETAL \& CONNECTIVE } \\
\text { TISSUE }\end{array}$ & $710-739$ & 2 & 9 & 4 & 42 \\
\hline -Arthropathies & $710-719$ & 2 & 5 & 1 & 18 \\
\hline -Dorsopathies & $720-724$ & 0 & 1 & 3 & 12 \\
\hline -Rheumatism, Excluding Back & 725-729 & 0 & 3 & 0 & 12 \\
\hline CONGENITAL ANOMALIES & $740-759$ & 0 & 0 & 0 & 1 \\
\hline $\begin{array}{l}\text { SYMPTOMS, SIGNS, \& ILL-DEFINED } \\
\text { CONDITIONS }\end{array}$ & 780-799 & 0 & 9 & 3 & 35 \\
\hline -Symptoms & 780-789 & 0 & 8 & 3 & 34 \\
\hline -Non-Specific Abnormal Findings & $790-796$ & 0 & 1 & 0 & 1 \\
\hline INJURY \& POISONING & $800-999$ & 3 & 7 & 1 & 49 \\
\hline -Fracture - Neck, Trunk & 805-809 & 0 & 0 & 0 & 7 \\
\hline -Fracture - Upper Limb & $810-819$ & 0 & 0 & 0 & 2 \\
\hline -Fracture - Lower Limb & $820-829$ & 0 & 1 & 0 & 4 \\
\hline -Dislocation & 830-839 & 2 & 5 & 0 & 12 \\
\hline -Sprains \& Strains - Other & $840-845,848$ & 1 & 0 & 0 & 8 \\
\hline -Open Wound - Upper Limb & 880-887 & 0 & 0 & 0 & 1 \\
\hline -Open Wound - Lower Limb & $890-897$ & 0 & 0 & 0 & 1 \\
\hline -Late Effects of Accident & 905-909 & 0 & 0 & 0 & 3 \\
\hline -Superficial Injury & 910-919 & 0 & 0 & 0 & 2 \\
\hline -Contusion & $920-924$ & 0 & 0 & 0 & 1 \\
\hline -Burns & 940-949 & 0 & 0 & 0 & 1 \\
\hline -Complications \& Unspecified Injuries & 958-959 & 0 & 0 & 1 & 1 \\
\hline -Toxic Effects - Non-medicinal & 980-989 & 0 & 1 & 0 & 1 \\
\hline -Complications of Surgical/Medical Care & 996-999 & 0 & 0 & 0 & 5 \\
\hline
\end{tabular}

(Continued)

*Only those diagnostic categories and gender/job category combinations with at least one occurrence appear in this table. 


\section{Kansas City Plant 2008}

Absence Data

Appendix I. Number of Diagnoses in Each Diagnostic Category by Gender and Job Category*

\begin{tabular}{|c|c|c|c|c|c|}
\hline & \multicolumn{4}{|c|}{ Men } \\
\hline & & \multicolumn{4}{|c|}{ Job Category } \\
\hline & & Professional & $\begin{array}{l}\text { Administrative } \\
\text { Support }\end{array}$ & Technical Support & Service \\
\hline Diagnostic Category & ICD-9-CM Code & \multirow[b]{2}{*}{1} & \multirow[b]{2}{*}{2} & \multirow[b]{2}{*}{0} & \multirow[b]{2}{*}{0} \\
\hline $\begin{array}{l}\text { HEALTH STATUS/HEALTH SERVICE } \\
\text { CONTACT }\end{array}$ & V01-V82 & & & & \\
\hline -Personal \& Family History & V10-V19 & 1 & 1 & 0 & 0 \\
\hline -Health Services Reproduction/Development & V20-V29 & 0 & 1 & 0 & 0 \\
\hline
\end{tabular}

*Only those diagnostic categories and gender/job category combinations with at least one occurrence appear in this table. 


\section{Kansas City Plant 2008}

Absence Data

Appendix I. Number of Diagnoses in Each Diagnostic Category by Gender and Job Category*

\begin{tabular}{|c|c|c|c|c|c|}
\hline & & \multicolumn{4}{|c|}{ Men } \\
\hline & & \multicolumn{3}{|c|}{ Job Category } & \multirow[b]{2}{*}{ TOTAL } \\
\hline & & Security and Fire & Crafts & Line Operators & \\
\hline Diagnostic Category & ICD-9-CM Code & & & & \\
\hline $\begin{array}{l}\text { HEALTH STATUS/HEALTH SERVICE } \\
\text { CONTACT }\end{array}$ & V01-V82 & 0 & 0 & 0 & 3 \\
\hline -Personal \& Family History & V10-V19 & 0 & 0 & 0 & 2 \\
\hline -Health Services Reproduction/Development & V20-V29 & 0 & 0 & 0 & 1 \\
\hline
\end{tabular}

\begin{tabular}{|c|c|c|c|c|c|c|c|c|}
\hline & \multicolumn{8}{|c|}{ Men } \\
\hline & \multicolumn{7}{|c|}{ Job Category } & \multirow[b]{2}{*}{ TOTAL } \\
\hline & Professional & $\begin{array}{c}\text { Administrative } \\
\text { Support }\end{array}$ & $\begin{array}{l}\text { Technical } \\
\text { Support }\end{array}$ & Service & $\begin{array}{l}\text { Security and } \\
\text { Fire }\end{array}$ & Crafts & $\begin{array}{c}\text { Line } \\
\text { Operators }\end{array}$ & \\
\hline Diagnostic Category & \multirow[b]{2}{*}{85} & \multirow[b]{2}{*}{31} & \multirow[b]{2}{*}{36} & \multirow[b]{2}{*}{16} & \multirow[b]{2}{*}{6} & \multirow[b]{2}{*}{46} & \multirow[b]{2}{*}{16} & \multirow[b]{2}{*}{236} \\
\hline Total & & & & & & & & \\
\hline
\end{tabular}

*Only those diagnostic categories and gender/job category combinations with at least one occurrence appear in this table. 


\section{Kansas City Plant 2008}

Absence Data

Appendix J. Total Number of Calendar Days Absent in Each Diagnostic Category by Gender and Job Category*

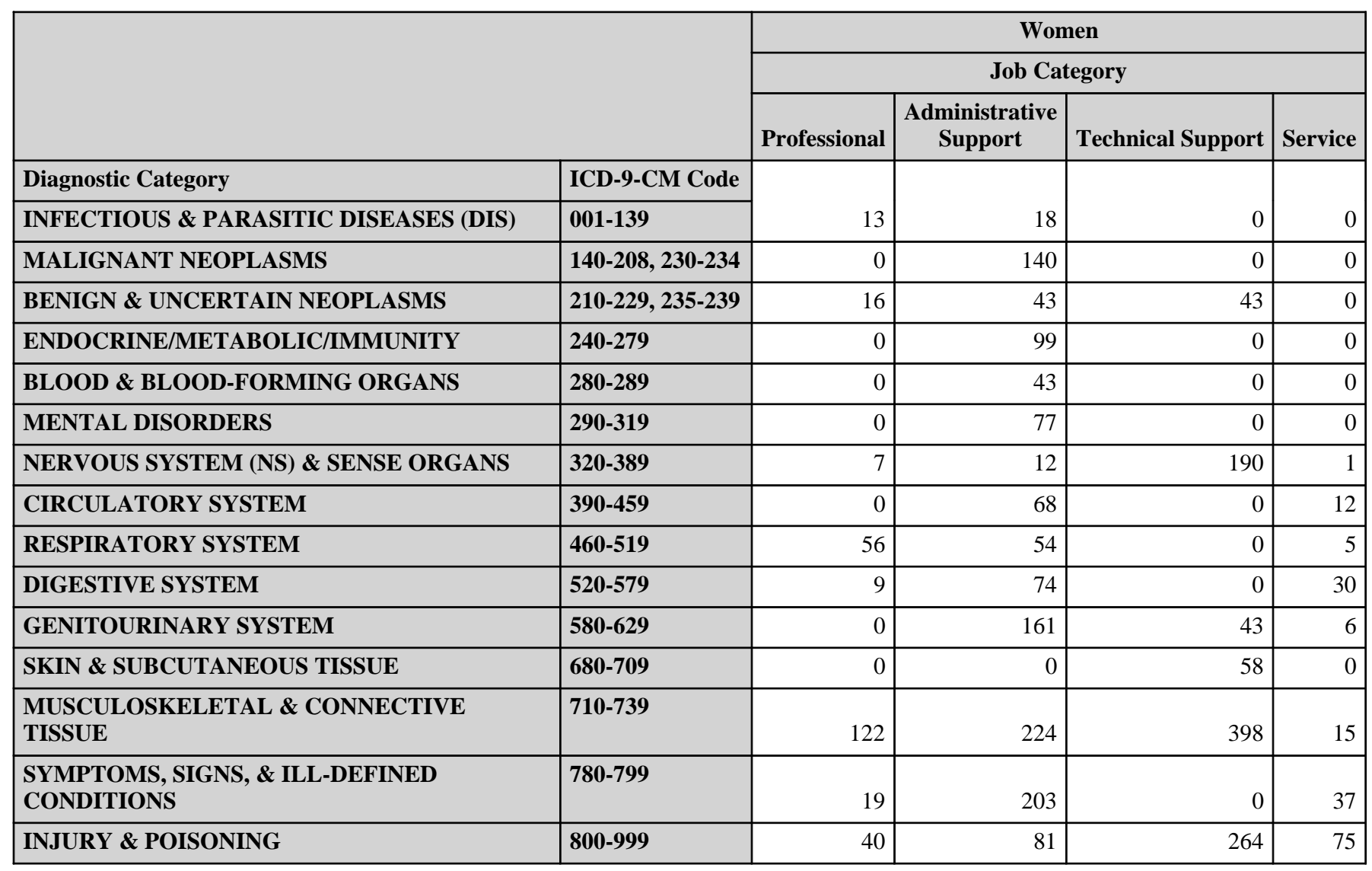

\footnotetext{
*Absences with >1 ICD-9-CM code in the same diagnostic category were counted only once. Only those diagnostic categories and gender/job category combinations with at least one occurrence appear in this table.
} 


\section{Kansas City Plant 2008}

Absence Data

Appendix J. Total Number of Calendar Days Absent in Each Diagnostic Category by Gender and Job Category*

\begin{tabular}{|c|c|c|c|c|c|}
\hline & & \multicolumn{4}{|c|}{ Women } \\
\hline & & \multicolumn{3}{|c|}{ Job Category } & \multirow[b]{2}{*}{ TOTAL } \\
\hline & & Security and Fire & Crafts & Line Operators & \\
\hline Diagnostic Category & ICD-9-CM Code & \multirow[b]{2}{*}{28} & \multirow[b]{2}{*}{0} & \multirow[b]{2}{*}{0} & \multirow[b]{2}{*}{59} \\
\hline INFECTIOUS \& PARASITIC DISEASES (DIS) & 001-139 & & & & \\
\hline MALIGNANT NEOPLASMS & $140-208,230-234$ & 0 & 0 & 240 & 380 \\
\hline BENIGN \& UNCERTAIN NEOPLASMS & 210-229, 235-239 & 0 & 0 & 0 & 102 \\
\hline ENDOCRINE/METABOLIC/IMMUNITY & $240-279$ & 0 & 0 & 0 & 99 \\
\hline BLOOD \& BLOOD-FORMING ORGANS & 280-289 & 0 & 0 & 0 & 43 \\
\hline MENTAL DISORDERS & 290-319 & 0 & 0 & 0 & 77 \\
\hline NERVOUS SYSTEM (NS) \& SENSE ORGANS & $320-389$ & 0 & 0 & 0 & 210 \\
\hline CIRCULATORY SYSTEM & $390-459$ & 0 & 0 & 82 & 162 \\
\hline RESPIRATORY SYSTEM & 460-519 & 0 & 6 & 0 & 121 \\
\hline DIGESTIVE SYSTEM & $520-579$ & 0 & 35 & 48 & 196 \\
\hline GENITOURINARY SYSTEM & $580-629$ & 0 & 0 & 34 & 244 \\
\hline SKIN \& SUBCUTANEOUS TISSUE & 680-709 & 0 & 0 & 5 & 63 \\
\hline $\begin{array}{l}\text { MUSCULOSKELETAL \& CONNECTIVE } \\
\text { TISSUE }\end{array}$ & $710-739$ & 28 & 0 & 52 & 839 \\
\hline $\begin{array}{l}\text { SYMPTOMS, SIGNS, \& ILL-DEFINED } \\
\text { CONDITIONS }\end{array}$ & 780-799 & 0 & 0 & 19 & 278 \\
\hline INJURY \& POISONING & 800-999 & 0 & 0 & 0 & 460 \\
\hline
\end{tabular}

\footnotetext{
*Absences with >1 ICD-9-CM code in the same diagnostic category were counted only once. Only those diagnostic categories and gender/job category combinations with at least one occurrence appear in this table.
} 


\section{Kansas City Plant 2008}

Absence Data

Appendix J. Total Number of Calendar Days Absent in Each Diagnostic Category by Gender and Job Category*

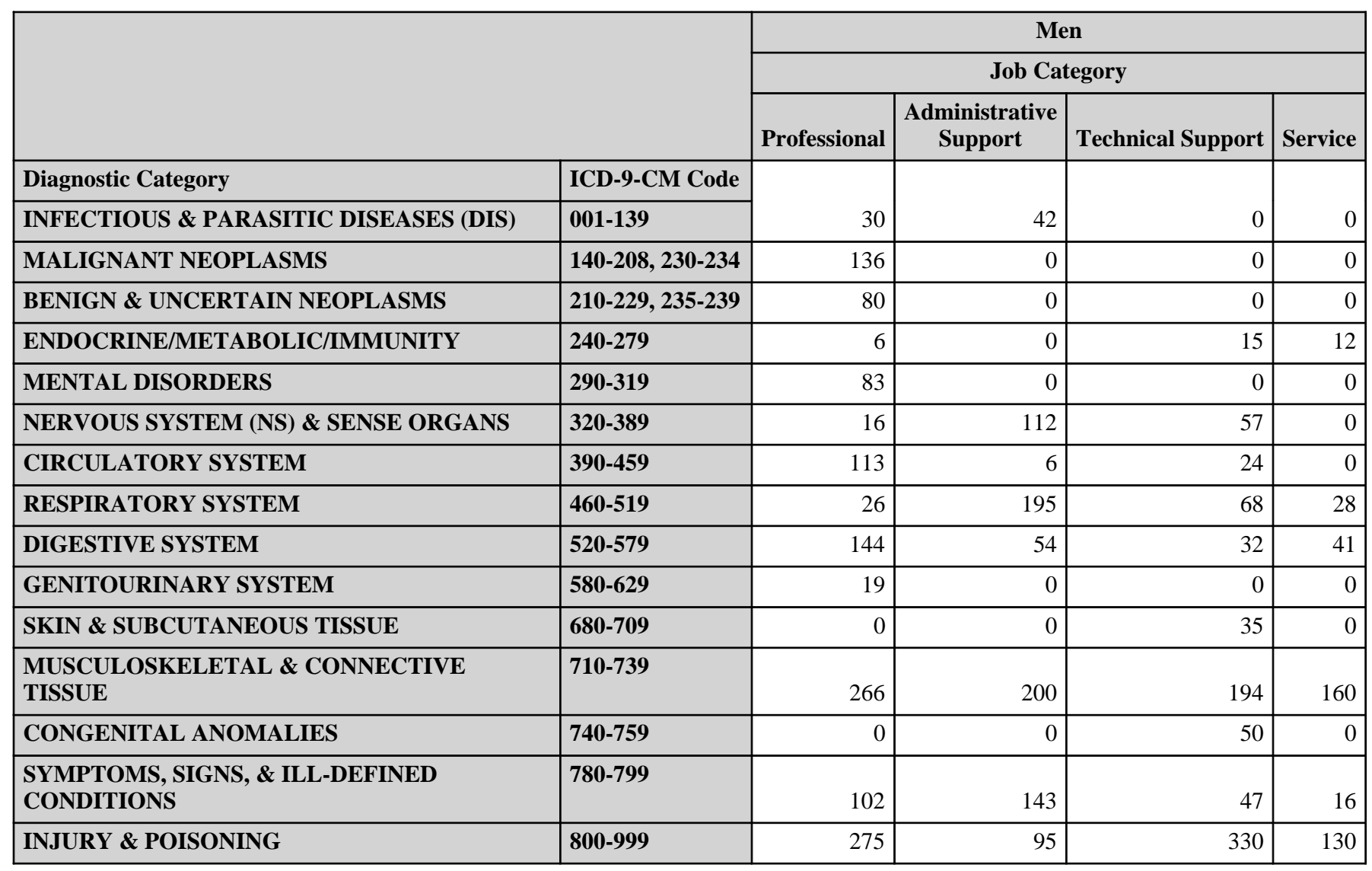

\footnotetext{
*Absences with >1 ICD-9-CM code in the same diagnostic category were counted only once. Only those diagnostic categories and gender/job category combinations with at least one occurrence appear in this table.
} 


\section{Kansas City Plant 2008}

Absence Data

Appendix J. Total Number of Calendar Days Absent in Each Diagnostic Category by Gender and Job Category*

\begin{tabular}{|c|c|c|c|c|c|}
\hline & & \multicolumn{4}{|c|}{ Men } \\
\hline & & \multicolumn{3}{|c|}{ Job Category } & \multirow[b]{2}{*}{ TOTAL } \\
\hline & & Security and Fire & Crafts & Line Operators & \\
\hline Diagnostic Category & ICD-9-CM Code & \multirow[b]{2}{*}{0} & \multirow[b]{2}{*}{167} & \multirow[b]{2}{*}{0} & \multirow[b]{2}{*}{239} \\
\hline INFECTIOUS \& PARASITIC DISEASES (DIS) & 001-139 & & & & \\
\hline MALIGNANT NEOPLASMS & $140-208,230-234$ & 0 & 167 & 21 & 324 \\
\hline BENIGN \& UNCERTAIN NEOPLASMS & 210-229, 235-239 & 0 & 0 & 2 & 82 \\
\hline ENDOCRINE/METABOLIC/IMMUNITY & $240-279$ & 0 & 0 & 0 & 33 \\
\hline MENTAL DISORDERS & 290-319 & 0 & 0 & 0 & 83 \\
\hline NERVOUS SYSTEM (NS) \& SENSE ORGANS & 320-389 & 0 & 1 & 0 & 186 \\
\hline CIRCULATORY SYSTEM & $390-459$ & 0 & 88 & 22 & 253 \\
\hline RESPIRATORY SYSTEM & $460-519$ & 0 & 19 & 16 & 352 \\
\hline DIGESTIVE SYSTEM & $520-579$ & 29 & 109 & 135 & 544 \\
\hline GENITOURINARY SYSTEM & $580-629$ & 0 & 148 & 10 & 177 \\
\hline SKIN \& SUBCUTANEOUS TISSUE & 680-709 & 0 & 0 & 0 & 35 \\
\hline $\begin{array}{l}\text { MUSCULOSKELETAL \& CONNECTIVE } \\
\text { TISSUE }\end{array}$ & $710-739$ & 61 & 231 & 105 & 1,217 \\
\hline CONGENITAL ANOMALIES & 740-759 & 0 & 0 & 0 & 50 \\
\hline $\begin{array}{l}\text { SYMPTOMS, SIGNS, \& ILL-DEFINED } \\
\text { CONDITIONS }\end{array}$ & 780-799 & 0 & 60 & 28 & 396 \\
\hline INJURY \& POISONING & 800-999 & 47 & 144 & 10 & 1,031 \\
\hline
\end{tabular}

\footnotetext{
*Absences with >1 ICD-9-CM code in the same diagnostic category were counted only once. Only those diagnostic categories and gender/job category combinations with at least one occurrence appear in this table.
} 


\section{Kansas City Plant 2008}

Absence Data

Appendix K. Age-Adjusted IIIness and Injury Rates by Diagnostic Category*

Part 1. Men

\begin{tabular}{|c|c|c|c|c|c|}
\hline & & $\begin{array}{l}\text { Number of } \\
\text { Diagnoses }\end{array}$ & $\begin{array}{c}\text { Age-Adjusted } \\
\text { Rate per } 1,000 * *\end{array}$ & \begin{tabular}{c|} 
Lower $95 \%$ \\
Confidence \\
Limit per 1,000
\end{tabular} & $\begin{array}{c}\text { Upper } 95 \% \\
\text { Confidence } \\
\text { Limit per } 1,000\end{array}$ \\
\hline Diagnostic Category & ICD-9-CM Code & & & & \\
\hline INFECTIOUS \& PARASITIC DISEASES (DIS) & 001-139 & 5 & 2.2 & 0.7 & 7.1 \\
\hline -Intestinal Infectious Dis & 001-009 & 1 & 0.2 & 0.0 & 1.5 \\
\hline -Other Bacterial Dis & 030-041 & 1 & 0.2 & 0.0 & 1.5 \\
\hline -Viral Dis with Exanthem & $050-057$ & 1 & 0.2 & 0.0 & 1.5 \\
\hline -Other Viral Dis \& Chlamydiae & 070-079 & 1 & 1.2 & 0.2 & 8.5 \\
\hline -Mycoses & $110-118$ & 1 & 0.4 & 0.1 & 2.9 \\
\hline MALIGNANT NEOPLASMS & $140-208,230-234$ & 11 & 3.3 & 1.8 & 6.2 \\
\hline -Digestive \& Peritoneal & 150-159 & 4 & 0.8 & 0.3 & 2.2 \\
\hline -Respiratory \& Intrathoracic & $160-165$ & 2 & 0.8 & 0.2 & 3.2 \\
\hline -Genitourinary & 179-189 & 1 & 0.2 & 0.0 & 1.5 \\
\hline -Other \& Unspecified Sites & 190, 193-199 & 2 & 0.6 & 0.1 & 2.6 \\
\hline -Nervous System & 191-192 & 2 & 0.8 & 0.2 & 3.4 \\
\hline BENIGN \& UNCERTAIN NEOPLASMS & 210-229, 235-239 & 3 & 0.6 & 0.2 & 1.9 \\
\hline ENDOCRINE/METABOLIC/IMMUNITY & $240-279$ & 3 & 1.0 & 0.3 & 3.3 \\
\hline -Thyroid Gland Disorders & $240-246$ & 1 & 0.2 & 0.0 & 1.5 \\
\hline -Other Endocrine Gland Dis & 250-259 & 1 & 0.4 & 0.1 & 3.0 \\
\hline -Other Metabolic \& Immunity Disorders & $270-279$ & 1 & 0.4 & 0.1 & 2.9 \\
\hline MENTAL DISORDERS & 290-319 & 6 & 4.8 & 2.0 & 11.8 \\
\hline -Psychoses & 290-299 & 2 & 2.4 & 0.6 & 9.6 \\
\hline -Non-Psychotic Disorders & $300-302,306-316$ & 4 & 2.5 & 0.8 & 7.5 \\
\hline NERVOUS SYSTEM (NS) \& SENSE ORGANS & 320-389 & 6 & 1.9 & 0.8 & 4.3 \\
\hline -Inflammatory Dis of Central NS & $320-326$ & 1 & 0.2 & 0.0 & 1.5 \\
\hline -Disorders of Peripheral NS & 350-359 & 4 & 1.5 & 0.5 & 4.0 \\
\hline -Disorders of Eye & $360-379$ & 1 & 0.2 & 0.0 & 1.5 \\
\hline CIRCULATORY SYSTEM & $390-459$ & 17 & 5.6 & 3.4 & 9.2 \\
\hline -Hypertensive Dis & $401-405$ & 1 & 0.4 & 0.1 & 2.9 \\
\hline -Ischemic Heart Dis & $410-414$ & 8 & 2.7 & 1.3 & 5.5 \\
\hline -Dis of Pulmonary Circulation & 415-417 & 1 & 0.2 & 0.0 & 1.5 \\
\hline -Other Heart Dis & $420-429$ & 4 & 1.5 & 0.5 & 4.0 \\
\hline -Cerebrovascular Dis & 430-438 & 1 & 0.4 & 0.1 & 2.9 \\
\hline -Dis of Veins, Lymphatics, Other & 451-459 & 2 & 0.4 & 0.1 & 1.7 \\
\hline RESPIRATORY SYSTEM & $460-519$ & 21 & 11.5 & 6.7 & 19.6 \\
\hline -Acute Respiratory Infections & $460-466$ & 1 & 0.4 & 0.1 & 3.0 \\
\hline -Other Dis Upper Respiratory Tract & \begin{tabular}{|l|}
$470-478$ \\
\end{tabular} & 7 & 6.3 & 2.8 & 14.4 \\
\hline -Pneumonia \& Influenza & $480-487$ & 12 & 4.3 & 2.1 & 8.8 \\
\hline
\end{tabular}

(Continued)

*Only those diagnostic categories with at least one occurrence appear in this table.

**Standardized to age distribution of 2000 U.S. population. 


\section{Kansas City Plant 2008}

Absence Data

Appendix K. Age-Adjusted IIIness and Injury Rates by Diagnostic Category*

Part 1. Men

\begin{tabular}{|c|c|c|c|c|c|}
\hline & & $\begin{array}{c}\text { Number of } \\
\text { Diagnoses }\end{array}$ & $\begin{array}{c}\text { Age-Adjusted } \\
\text { Rate per } 1,000^{* *}\end{array}$ & \begin{tabular}{c|} 
Lower $95 \%$ \\
Confidence \\
Limit per 1,000
\end{tabular} & $\begin{array}{c}\text { Upper } 95 \% \\
\text { Confidence } \\
\text { Limit per 1,000 }\end{array}$ \\
\hline Diagnostic Category & ICD-9-CM Code & & & & \\
\hline -Chronic Obstructive Dis & $490-496$ & 1 & 0.4 & 0.1 & 2.9 \\
\hline DIGESTIVE SYSTEM & 520-579 & 29 & 10.1 & 6.6 & 15.5 \\
\hline -Esophagus, Stomach, Duodenum & 530-537 & 1 & 0.4 & 0.1 & 3.0 \\
\hline -Appendicitis & $540-543$ & 1 & 0.4 & 0.1 & 3.0 \\
\hline -Hernia & $\begin{array}{l}550-553 \\
\end{array}$ & 12 & 3.9 & 2.2 & 7.1 \\
\hline -Enteritis, Colitis & $555-558$ & 1 & 1.4 & 0.2 & 9.7 \\
\hline -Other Intestinal Dis & 560-569 & 5 & 1.3 & 0.5 & 3.2 \\
\hline -Other Digestive Dis & 570-579 & 9 & 2.7 & 1.4 & 5.4 \\
\hline GENITOURINARY SYSTEM & $580-629$ & 4 & 2.6 & 0.8 & 8.3 \\
\hline -Other Urinary Dis & 590-599 & 3 & 2.2 & 0.6 & 8.2 \\
\hline -Male Genital Organ Dis & $600-608$ & 1 & 0.4 & 0.1 & 2.9 \\
\hline SKIN \& SUBCUTANEOUS TISSUE & 680-709 & 1 & 0.2 & 0.0 & 1.5 \\
\hline -Infections & $680-686$ & 1 & 0.2 & 0.0 & 1.5 \\
\hline $\begin{array}{l}\text { MUSCULOSKELETAL \& CONNECTIVE } \\
\text { TISSUE }\end{array}$ & 710-739 & 42 & 13.5 & 9.5 & 19.2 \\
\hline -Arthropathies & 710-719 & 18 & 7.3 & 4.3 & 12.6 \\
\hline -Dorsopathies & 720-724 & 12 & 3.1 & 1.7 & 5.6 \\
\hline -Rheumatism, Excluding Back & 725-729 & 12 & 3.1 & 1.7 & 5.6 \\
\hline CONGENITAL ANOMALIES & 740-759 & 1 & 0.4 & 0.1 & 3.0 \\
\hline $\begin{array}{l}\text { SYMPTOMS, SIGNS, \& ILL-DEFINED } \\
\text { CONDITIONS }\end{array}$ & 780-799 & 35 & 17.5 & 11.6 & 26.4 \\
\hline -Symptoms & 780-789 & 34 & 17.3 & 11.4 & 26.2 \\
\hline -Non-Specific Abnormal Findings & 790-796 & 1 & 0.2 & 0.0 & 1.5 \\
\hline INJURY \& POISONING & $800-999$ & 49 & 19.2 & 13.6 & 27.3 \\
\hline -Fracture - Skull & 800-804 & 7 & 2.1 & 1.0 & 4.6 \\
\hline -Fracture - Upper Limb & $810-819$ & 2 & 0.8 & 0.2 & 3.4 \\
\hline -Fracture - Lower Limb & $820-829$ & 4 & 1.7 & 0.6 & 4.5 \\
\hline -Dislocation & 830-839 & 12 & 3.1 & 1.7 & 5.6 \\
\hline -Sprains \& Strains - Other & $840-845,848$ & 8 & 4.4 & 1.8 & 10.6 \\
\hline -Open Wound - Head, Neck, Trunk & $870-879$ & 2 & 1.8 & 0.4 & 8.5 \\
\hline -Late Effects of Accident & 905-909 & 3 & 0.8 & 0.3 & 2.8 \\
\hline -Superficial Injury & 910-919 & 2 & 0.4 & 0.1 & 1.7 \\
\hline -Contusion & 920-924 & 1 & 1.4 & 0.2 & 9.7 \\
\hline -Burns & 940-949 & 1 & 0.4 & 0.1 & 3.0 \\
\hline -Complications \& Unspecified Injuries & 958-959 & 1 & 0.4 & 0.1 & 3.0 \\
\hline -Toxic Effects - Non-medicinal & $980-989$ & 1 & 0.2 & 0.0 & 1.5 \\
\hline
\end{tabular}

(Continued)

*Only those diagnostic categories with at least one occurrence appear in this table.

**Standardized to age distribution of 2000 U.S. population. 
Kansas City Plant 2008

Absence Data

Appendix K. Age-Adjusted Illness and Injury Rates by Diagnostic Category*

Part 1. Men

\begin{tabular}{|c|c|c|c|c|c|}
\hline & & $\begin{array}{l}\text { Number of } \\
\text { Diagnoses }\end{array}$ & $\begin{array}{c}\text { Age-Adjusted } \\
\text { Rate per } 1,000^{* *}\end{array}$ & $\begin{array}{c}\text { Lower } 95 \% \\
\text { Confidence } \\
\text { Limit per } 1,000\end{array}$ & $\begin{array}{c}\text { Upper } 95 \% \\
\text { Confidence } \\
\text { Limit per 1,000 }\end{array}$ \\
\hline Diagnostic Category & ICD-9-CM Code & \multirow[b]{2}{*}{5} & \multirow[b]{2}{*}{1.7} & \multirow[b]{2}{*}{0.7} & \multirow[b]{2}{*}{4.2} \\
\hline -Complications of Surgical/Medical Care & 996-999 & & & & \\
\hline Total & & 233 & 94.6 & 80.6 & 110.9 \\
\hline
\end{tabular}

* Only those diagnostic categories with at least one occurrence appear in this table.

**Standardized to age distribution of 2000 U.S. population. 


\section{Kansas City Plant 2008}

Absence Data

Appendix K. Age-Adjusted Illness and Injury Rates by Diagnostic Category*

Part 2. Women

\begin{tabular}{|c|c|c|c|c|c|}
\hline & & $\begin{array}{l}\text { Number of } \\
\text { Diagnoses }\end{array}$ & $\begin{array}{c}\text { Age-Adjusted } \\
\text { Rate per } 1,000 * *\end{array}$ & $\begin{array}{c}\text { Lower } 95 \% \\
\text { Confidence } \\
\text { Limit per 1,000 }\end{array}$ & $\begin{array}{c}\text { Upper } 95 \% \\
\text { Confidence } \\
\text { Limit per } 1,000\end{array}$ \\
\hline Diagnostic Category & ICD-9-CM Code & & & & \\
\hline INFECTIOUS \& PARASITIC DISEASES (DIS) & 001-139 & 4 & 4.2 & 1.5 & 12.2 \\
\hline -Other Bacterial Dis & 030-041 & 1 & 0.6 & 0.1 & 4.4 \\
\hline -Viral Dis with Exanthem & 050-057 & 1 & 1.5 & 0.2 & 10.6 \\
\hline -Other Viral Dis \& Chlamydiae & 070-079 & 1 & 1.5 & 0.2 & 10.6 \\
\hline -Mycoses & $110-118$ & 1 & 0.6 & 0.1 & 4.4 \\
\hline MALIGNANT NEOPLASMS & $140-208,230-234$ & 9 & 8.0 & 3.9 & 16.2 \\
\hline -Digestive \& Peritoneal & $150-159$ & 1 & 1.3 & 0.2 & 8.9 \\
\hline -Breast & 174-175 & 1 & 1.5 & 0.2 & 10.6 \\
\hline -Genitourinary & $179-189$ & 1 & 1.5 & 0.2 & 10.6 \\
\hline -Other \& Unspecified Sites & $190,193-199$ & 6 & 3.7 & 1.7 & 8.3 \\
\hline BENIGN \& UNCERTAIN NEOPLASMS & $210-229,235-239$ & 3 & 6.8 & 1.8 & 25.7 \\
\hline ENDOCRINE/METABOLIC/IMMUNITY & $240-279$ & 3 & 2.5 & 0.7 & 8.3 \\
\hline -Thyroid Gland Disorders & $240-246$ & 1 & 1.3 & 0.2 & 8.9 \\
\hline -Other Endocrine Gland Dis & $250-259$ & 1 & 0.6 & 0.1 & 4.4 \\
\hline -Other Metabolic \& Immunity Disorders & 270-279 & 1 & 0.6 & 0.1 & 4.4 \\
\hline BLOOD \& BLOOD-FORMING ORGANS & 280-289 & 1 & 1.3 & 0.2 & 8.9 \\
\hline MENTAL DISORDERS & 290-319 & 1 & 0.6 & 0.1 & 4.4 \\
\hline -Non-Psychotic Disorders & $300-302,306-316$ & 1 & 0.6 & 0.1 & 4.4 \\
\hline NERVOUS SYSTEM (NS) \& SENSE ORGANS & 320-389 & 7 & 7.1 & 3.2 & 15.6 \\
\hline -Hereditary/Degenerative Central NS Dis & 330-337 & 1 & 0.6 & 0.1 & 4.4 \\
\hline -Other Disorders of Central NS & 340-349 & 2 & 2.5 & 0.6 & 10.0 \\
\hline -Disorders of Peripheral NS & $350-359$ & 2 & 2.1 & 0.5 & 9.5 \\
\hline -Disorders of Eye & $360-379$ & 2 & 1.9 & 0.4 & 8.1 \\
\hline CIRCULATORY SYSTEM & $390-459$ & 5 & 6.1 & 2.5 & 15.1 \\
\hline -Ischemic Heart Dis & $410-414$ & 1 & 1.5 & 0.2 & 10.6 \\
\hline -Dis of Pulmonary Circulation & $415-417$ & 1 & 1.3 & 0.2 & 8.9 \\
\hline -Other Heart Dis & $420-429$ & 2 & 2.7 & 0.7 & 11.0 \\
\hline -Dis of Arteries \& Capillaries & 440-448 & 1 & 0.6 & 0.1 & 4.4 \\
\hline RESPIRATORY SYSTEM & $460-519$ & 13 & 16.1 & 8.1 & 32.2 \\
\hline -Acute Respiratory Infections & $460-466$ & 5 & 5.0 & 2.0 & 12.5 \\
\hline -Other Dis Upper Respiratory Tract & $470-478$ & 2 & 5.0 & 0.9 & 28.4 \\
\hline -Pneumonia \& Influenza & $480-487$ & 4 & 4.0 & 1.4 & 11.4 \\
\hline -Lung Dis from External Agents & $500-508$ & 1 & 0.6 & 0.1 & 4.4 \\
\hline -Other Respiratory Dis & \begin{tabular}{|l|}
$510-519$ \\
\end{tabular} & 1 & 1.5 & 0.2 & 10.6 \\
\hline DIGESTIVE SYSTEM & $520-579$ & 7 & 6.5 & 2.9 & 14.4 \\
\hline
\end{tabular}

(Continued)

* Only those diagnostic categories with at least one occurrence appear in this table.

**Standardized to age distribution of 2000 U.S. population. 


\section{Kansas City Plant 2008}

Absence Data

Appendix K. Age-Adjusted IIIness and Injury Rates by Diagnostic Category*

Part 2. Women

\begin{tabular}{|c|c|c|c|c|c|}
\hline & & $\begin{array}{l}\text { Number of } \\
\text { Diagnoses }\end{array}$ & $\begin{array}{c}\text { Age-Adjusted } \\
\text { Rate per } 1,000 * *\end{array}$ & $\begin{array}{c}\text { Lower } 95 \% \\
\text { Confidence } \\
\text { Limit per 1,000 }\end{array}$ & $\begin{array}{c}\text { Upper } 95 \% \\
\text { Confidence } \\
\text { Limit per } 1,000\end{array}$ \\
\hline Diagnostic Category & ICD-9-CM Code & & & & \\
\hline -Appendicitis & $540-543$ & 1 & 0.6 & 0.1 & 4.4 \\
\hline -Hernia & $550-553$ & 2 & 2.1 & 0.5 & 9.5 \\
\hline -Other Digestive Dis & $570-579$ & 4 & 3.7 & 1.3 & 10.5 \\
\hline GENITOURINARY SYSTEM & $580-629$ & 9 & 10.3 & 5.2 & 20.1 \\
\hline -Other Urinary Dis & $590-599$ & 2 & 2.7 & 0.7 & 11.0 \\
\hline -Breast Disorders & $610-611$ & 2 & 2.5 & 0.6 & 10.0 \\
\hline -Other Female Disorders & $617-629$ & 5 & 5.0 & 2.0 & 12.5 \\
\hline SKIN \& SUBCUTANEOUS TISSUE & 680-709 & 2 & 5.0 & 0.9 & 28.4 \\
\hline -Infections & $680-686$ & 2 & 5.0 & 0.9 & 28.4 \\
\hline $\begin{array}{l}\text { MUSCULOSKELETAL \& CONNECTIVE } \\
\text { TISSUE }\end{array}$ & $710-739$ & 23 & 33.7 & 19.4 & 58.7 \\
\hline -Arthropathies & $710-719$ & 8 & 7.1 & 3.4 & 15.0 \\
\hline -Dorsopathies & $720-724$ & 5 & 8.1 & 2.6 & 26.0 \\
\hline -Rheumatism, Excluding Back & 725-729 & 7 & 16.6 & 6.7 & 41.2 \\
\hline -Other Dis \& Acquired Deformities & $730-739$ & 3 & 1.9 & 0.6 & 5.8 \\
\hline $\begin{array}{l}\text { SYMPTOMS, SIGNS, \& ILL-DEFINED } \\
\text { CONDITIONS }\end{array}$ & $780-799$ & 15 & 19.5 & 9.7 & 39.0 \\
\hline -Symptoms & $780-789$ & 13 & 17.6 & 8.3 & 37.4 \\
\hline -Non-Specific Abnormal Findings & $790-796$ & 2 & 1.9 & 0.4 & 8.1 \\
\hline INJURY \& POISONING & $800-999$ & 10 & 9.0 & 4.6 & 17.4 \\
\hline -Fracture - Skull & $800-804$ & 1 & 0.6 & 0.1 & 4.4 \\
\hline -Dislocation & 830-839 & 3 & 1.9 & 0.6 & 5.8 \\
\hline -Intracranial Injury & $850-854$ & 1 & 0.6 & 0.1 & 4.4 \\
\hline -Open Wound - Head, Neck, Trunk & $870-879$ & 2 & 1.9 & 0.4 & 8.1 \\
\hline -Foreign Body Entering Orifice & 930-939 & 1 & 1.3 & 0.2 & 8.9 \\
\hline -Complications \& Unspecified Injuries & 958-959 & 1 & 1.5 & 0.2 & 10.6 \\
\hline -Complications of Surgical/Medical Care & 996-999 & 1 & 1.3 & 0.2 & 8.9 \\
\hline Total & & 112 & 136.6 & 107.7 & 173.2 \\
\hline
\end{tabular}

* Only those diagnostic categories with at least one occurrence appear in this table.

**Standardized to age distribution of 2000 U.S. population. 


\section{Kansas City Plant 2008}

\section{Absence Data}

Appendix K. Age-Adjusted IIIness and Injury Rates by Diagnostic Category*

Part 3. Men and Women

\begin{tabular}{|c|c|c|c|c|c|}
\hline & & $\begin{array}{c}\text { Number of } \\
\text { Diagnoses }\end{array}$ & $\begin{array}{c}\text { Age-Adjusted } \\
\text { Rate per } 1,000^{* *}\end{array}$ & \begin{tabular}{|c|} 
Lower $95 \%$ \\
Confidence \\
Limit per 1,000
\end{tabular} & $\begin{array}{c}\text { Upper } 95 \% \\
\text { Confidence } \\
\text { Limit per 1,000 }\end{array}$ \\
\hline Diagnostic Category & ICD-9-CM Code & & & & \\
\hline INFECTIOUS \& PARASITIC DISEASES (DIS) & 001-139 & 9 & 2.7 & 1.2 & 6.2 \\
\hline -Intestinal Infectious Dis & 001-009 & 1 & 0.2 & 0.0 & 1.1 \\
\hline -Other Bacterial Dis & 030-041 & 2 & 0.3 & 0.1 & 1.3 \\
\hline -Viral Dis with Exanthem & 050-057 & 2 & 0.5 & 0.1 & 2.1 \\
\hline -Other Viral Dis \& Chlamydiae & 070-079 & 2 & 1.3 & 0.3 & 5.9 \\
\hline -Mycoses & 110-118 & 2 & 0.5 & 0.1 & 2.1 \\
\hline MALIGNANT NEOPLASMS & $140-208,230-234$ & 20 & 4.5 & 2.8 & 7.1 \\
\hline -Digestive \& Peritoneal & $150-159$ & 5 & 0.9 & 0.4 & 2.4 \\
\hline -Respiratory \& Intrathoracic & $160-165$ & 2 & 0.7 & 0.2 & 2.6 \\
\hline -Breast & 174-175 & 1 & 0.3 & 0.0 & 2.3 \\
\hline -Genitourinary & 179-189 & 2 & 0.5 & 0.1 & 2.1 \\
\hline -Other \& Unspecified Sites & 190, 193-199 & 8 & 1.4 & 0.7 & 3.0 \\
\hline -Nervous System & 191-192 & 2 & 0.6 & 0.2 & 2.5 \\
\hline BENIGN \& UNCERTAIN NEOPLASMS & 210-229, 235-239 & 6 & 2.1 & 0.7 & 6.1 \\
\hline ENDOCRINE/METABOLIC/IMMUNITY & $240-279$ & 6 & 1.4 & 0.6 & 3.3 \\
\hline -Thyroid Gland Disorders & $240-246$ & 2 & 0.5 & 0.1 & 2.0 \\
\hline -Other Endocrine Gland Dis & 250-259 & 2 & 0.5 & 0.1 & 2.0 \\
\hline -Other Metabolic \& Immunity Disorders & 270-279 & 2 & 0.5 & 0.1 & 2.1 \\
\hline BLOOD \& BLOOD-FORMING ORGANS & $280-289$ & 1 & 0.3 & 0.0 & 2.2 \\
\hline MENTAL DISORDERS & 290-319 & 7 & 3.9 & 1.7 & 9.2 \\
\hline -Psychoses & 290-299 & 2 & 1.9 & 0.5 & 7.5 \\
\hline -Non-Psychotic Disorders & $300-302,306-316$ & 5 & 2.0 & 0.7 & 5.8 \\
\hline NERVOUS SYSTEM (NS) \& SENSE ORGANS & 320-389 & 13 & 3.2 & 1.8 & 5.6 \\
\hline -Inflammatory Dis of Central NS & $320-326$ & 1 & 0.2 & 0.0 & 1.1 \\
\hline -Hereditary/Degenerative Central NS Dis & 330-337 & 1 & 0.2 & 0.0 & 1.1 \\
\hline -Other Disorders of Central NS & 340-349 & 2 & 0.6 & 0.2 & 2.5 \\
\hline -Disorders of Peripheral NS & 350-359 & 6 & 1.6 & 0.7 & 3.7 \\
\hline -Disorders of Eye & $360-379$ & 3 & 0.6 & 0.2 & 2.1 \\
\hline CIRCULATORY SYSTEM & $390-459$ & 22 & 5.8 & 3.7 & 8.9 \\
\hline -Hypertensive Dis & $401-405$ & 1 & 0.3 & 0.0 & 2.3 \\
\hline -Ischemic Heart Dis & $410-414$ & 9 & 2.4 & 1.2 & 4.8 \\
\hline -Dis of Pulmonary Circulation & $415-417$ & 2 & 0.5 & 0.1 & 2.0 \\
\hline -Other Heart Dis & 420-429 & 6 & 1.7 & 0.8 & 3.9 \\
\hline -Cerebrovascular Dis & $430-438$ & 1 & 0.3 & 0.0 & 2.3 \\
\hline -Dis of Arteries \& Capillaries & $440-448$ & 1 & 0.2 & 0.0 & 1.1 \\
\hline
\end{tabular}

(Continued)

* Only those diagnostic categories with at least one occurrence appear in this table.

**Standardized to age distribution of 2000 U.S. population. 


\section{Kansas City Plant 2008}

\section{Absence Data}

Appendix K. Age-Adjusted IIIness and Injury Rates by Diagnostic Category*

Part 3. Men and Women

\begin{tabular}{|c|c|c|c|c|c|}
\hline & & $\begin{array}{l}\text { Number of } \\
\text { Diagnoses }\end{array}$ & $\begin{array}{l}\text { Age-Adjusted } \\
\text { Rate per } 1,000 * *\end{array}$ & $\begin{array}{c}\text { Lower } 95 \% \\
\text { Confidence } \\
\text { Limit per } 1,000\end{array}$ & $\begin{array}{c}\text { Upper } 95 \% \\
\text { Confidence } \\
\text { Limit per 1,000 }\end{array}$ \\
\hline Diagnostic Category & ICD-9-CM Code & \multirow[b]{2}{*}{2} & \multirow[b]{2}{*}{0.3} & \multirow[b]{2}{*}{0.1} & \multirow[b]{2}{*}{1.3} \\
\hline -Dis of Veins, Lymphatics, Other & 451-459 & & & & \\
\hline RESPIRATORY SYSTEM & $460-519$ & 34 & 12.6 & 8.3 & 19.3 \\
\hline -Acute Respiratory Infections & $460-466$ & 6 & 1.6 & 0.7 & 3.6 \\
\hline -Other Dis Upper Respiratory Tract & $470-478$ & 9 & 6.0 & 2.9 & 12.5 \\
\hline -Pneumonia \& Influenza & $480-487$ & 16 & 4.3 & 2.3 & $\overline{7.8}$ \\
\hline -Chronic Obstructive Dis & $490-496$ & 1 & 0.3 & 0.0 & 2.3 \\
\hline -Lung Dis from External Agents & $500-508$ & 1 & 0.2 & 0.0 & 1.1 \\
\hline -Other Respiratory Dis & $510-519$ & 1 & 0.3 & 0.0 & 2.3 \\
\hline DIGESTIVE SYSTEM & $520-579$ & 36 & 9.3 & 6.3 & 13.5 \\
\hline -Esophagus, Stomach, Duodenum & $530-537$ & 1 & 0.3 & 0.0 & 2.2 \\
\hline -Appendicitis & $540-543$ & 2 & 0.5 & 0.1 & 2.0 \\
\hline -Hernia & $550-553$ & 14 & 3.5 & 2.0 & 6.1 \\
\hline -Enteritis, Colitis & $555-558$ & 1 & 1.0 & 0.1 & 7.2 \\
\hline -Other Intestinal Dis & $560-569$ & 5 & 0.9 & 0.4 & 2.4 \\
\hline -Other Digestive Dis & $570-579$ & 13 & 3.0 & 1.7 & 5.3 \\
\hline GENITOURINARY SYSTEM & $580-629$ & 13 & 4.5 & 2.4 & 8.5 \\
\hline -Other Urinary Dis & $590-599$ & 5 & 2.3 & 0.8 & 6.4 \\
\hline -Male Genital Organ Dis & $600-608$ & 1 & 0.3 & 0.0 & 2.3 \\
\hline -Breast Disorders & $610-611$ & 2 & 0.6 & 0.2 & 2.5 \\
\hline -Other Female Disorders & $617-629$ & 5 & 1.3 & 0.5 & 3.1 \\
\hline SKIN \& SUBCUTANEOUS TISSUE & 680-709 & 3 & 1.2 & 0.3 & 5.6 \\
\hline -Infections & $680-686$ & 3 & 1.2 & 0.3 & $\overline{5.6}$ \\
\hline $\begin{array}{l}\text { MUSCULOSKELETAL \& CONNECTIVE } \\
\text { TISSUE }\end{array}$ & $710-739$ & 65 & 18.4 & 13.6 & 25.0 \\
\hline -Arthropathies & 710-719 & 26 & 7.4 & 4.7 & 11.6 \\
\hline -Dorsopathies & $720-724$ & 17 & 4.3 & 2.4 & 7.7 \\
\hline -Rheumatism, Excluding Back & 725-729 & 19 & 6.3 & 3.5 & 11.5 \\
\hline -Other Dis \& Acquired Deformities & 730-739 & 3 & 0.5 & 0.2 & 1.5 \\
\hline CONGENITAL ANOMALIES & 740-759 & 1 & 0.3 & 0.0 & 2.2 \\
\hline $\begin{array}{l}\text { SYMPTOMS, SIGNS, \& ILL-DEFINED } \\
\text { CONDITIONS }\end{array}$ & $780-799$ & 50 & 18.2 & 12.8 & 25.8 \\
\hline -Symptoms & 780-789 & 47 & 17.6 & 12.3 & 25.1 \\
\hline -Non-Specific Abnormal Findings & $790-796$ & 3 & 0.6 & 0.2 & 2.1 \\
\hline INJURY \& POISONING & $800-999$ & 59 & 16.8 & 12.3 & 23.0 \\
\hline -Fracture - Skull & $800-804$ & 8 & 1.7 & 0.8 & 3.6 \\
\hline -Fracture - Upper Limb & $810-819$ & 2 & 0.6 & 0.2 & 2.5 \\
\hline
\end{tabular}

(Continued)

*Only those diagnostic categories with at least one occurrence appear in this table.

**Standardized to age distribution of 2000 U.S. population. 


\section{Kansas City Plant 2008}

Absence Data

Appendix K. Age-Adjusted Illness and Injury Rates by Diagnostic Category*

Part 3. Men and Women

\begin{tabular}{|c|c|c|c|c|c|}
\hline & & $\begin{array}{l}\text { Number of } \\
\text { Diagnoses }\end{array}$ & $\begin{array}{c}\text { Age-Adjusted } \\
\text { Rate per } 1,000 * *\end{array}$ & $\begin{array}{c}\text { Lower } 95 \% \\
\text { Confidence } \\
\text { Limit per 1,000 }\end{array}$ & $\begin{array}{c}\text { Upper } 95 \% \\
\text { Confidence } \\
\text { Limit per 1,000 }\end{array}$ \\
\hline Diagnostic Category & ICD-9-CM Code & \multirow[b]{2}{*}{4} & \multirow[b]{2}{*}{1.3} & \multirow[b]{2}{*}{0.5} & \multirow[b]{2}{*}{3.4} \\
\hline -Fracture - Lower Limb & $820-829$ & & & & \\
\hline -Dislocation & $830-839$ & 15 & 2.9 & 1.7 & 4.9 \\
\hline -Sprains \& Strains - Other & $840-845,848$ & 8 & 3.4 & 1.4 & 8.1 \\
\hline -Intracranial Injury & $850-854$ & 1 & 0.2 & 0.0 & 1.1 \\
\hline -Open Wound - Head, Neck, Trunk & $870-879$ & 4 & 1.8 & 0.5 & 6.1 \\
\hline -Late Effects of Accident & 905-909 & 3 & 0.6 & 0.2 & 2.1 \\
\hline -Superficial Injury & $910-919$ & 2 & 0.3 & 0.1 & 1.3 \\
\hline -Contusion & $920-924$ & 1 & 1.0 & 0.1 & 7.2 \\
\hline -Foreign Body Entering Orifice & 930-939 & 1 & 0.3 & 0.0 & 2.2 \\
\hline -Burns & $940-949$ & 1 & 0.3 & 0.0 & 2.2 \\
\hline -Complications \& Unspecified Injuries & 958-959 & 2 & 0.6 & 0.2 & 2.6 \\
\hline -Toxic Effects - Non-medicinal & 980-989 & 1 & 0.2 & 0.0 & 1.1 \\
\hline -Complications of Surgical/Medical Care & 996-999 & 6 & 1.6 & 0.7 & 3.6 \\
\hline Total & & 345 & 105.3 & 92.4 & 120.0 \\
\hline
\end{tabular}

* Only those diagnostic categories with at least one occurrence appear in this table.

**Standardized to age distribution of 2000 U.S. population. 


\section{Kansas City Plant 2008}

OSHA Data

Appendix L. Number of Workers with at Least One OSHA Event by Gender, Age, and Job Category*

\begin{tabular}{|c|c|c|c|c|c|c|c|}
\hline \multirow{3}{*}{ Job Category } & \multicolumn{3}{|c|}{ Women } & \multicolumn{3}{|c|}{ Men } & \multirow[b]{3}{*}{ TOTAL } \\
\hline & \multicolumn{2}{|c|}{ Age Group } & \multirow[b]{2}{*}{ TOTAL } & \multicolumn{2}{|c|}{ Age Group } & \multirow[b]{2}{*}{ TOTAL } & \\
\hline & $40-49$ & $50+$ & & $40-49$ & $50+$ & & \\
\hline Administrative Support & 0 & 1 & 1 & 0 & 0 & 0 & 1 \\
\hline Technical Support & 0 & 0 & 0 & 1 & 1 & 2 & 2 \\
\hline Service & 1 & 1 & 2 & 0 & 1 & 1 & 3 \\
\hline Security and Fire & 0 & 0 & 0 & 0 & 1 & 1 & 1 \\
\hline Crafts & 0 & 0 & 0 & 0 & 5 & 5 & 5 \\
\hline Line Operators & 1 & 0 & 1 & 0 & 0 & 0 & 1 \\
\hline TOTAL & 2 & 2 & 4 & 1 & 8 & 9 & 13 \\
\hline
\end{tabular}

*Only those job categories and gender/age combinations with at least one OSHA event appear in this table.

Appendix M. Total Number of Workdays Lost or with Restricted Activity from OSHA Events by Gender and Age

\begin{tabular}{|c|c|c|c|c|c|c|c|c|c|}
\hline \multirow[b]{2}{*}{ Age Group } & \multicolumn{3}{|c|}{ Women } & \multicolumn{3}{|c|}{ Men } & \multicolumn{3}{|c|}{ TOTAL } \\
\hline & $\begin{array}{c}\text { Number of } \\
\text { Events }\end{array}$ & \begin{tabular}{|c} 
Days \\
Restricted
\end{tabular} & Days Lost & $\begin{array}{l}\text { Number of } \\
\text { Events }\end{array}$ & $\begin{array}{c}\text { Days } \\
\text { Restricted }\end{array}$ & Days Lost & \begin{tabular}{|c|} 
Number of \\
Events
\end{tabular} & $\begin{array}{c}\text { Days } \\
\text { Restricted }\end{array}$ & Days Lost \\
\hline $40-49$ & 2 & 1 & 0 & 1 & 26 & 20 & 3 & 27 & 20 \\
\hline $50+$ & 2 & 1 & 1 & 8 & 270 & 155 & 10 & 271 & 156 \\
\hline TOTAL & 4 & 2 & 1 & 9 & 296 & 175 & 13 & 298 & 176 \\
\hline
\end{tabular}

Appendix N. Total Number of Workdays Lost or with Restricted Activity from OSHA Events by Gender and Job Category*

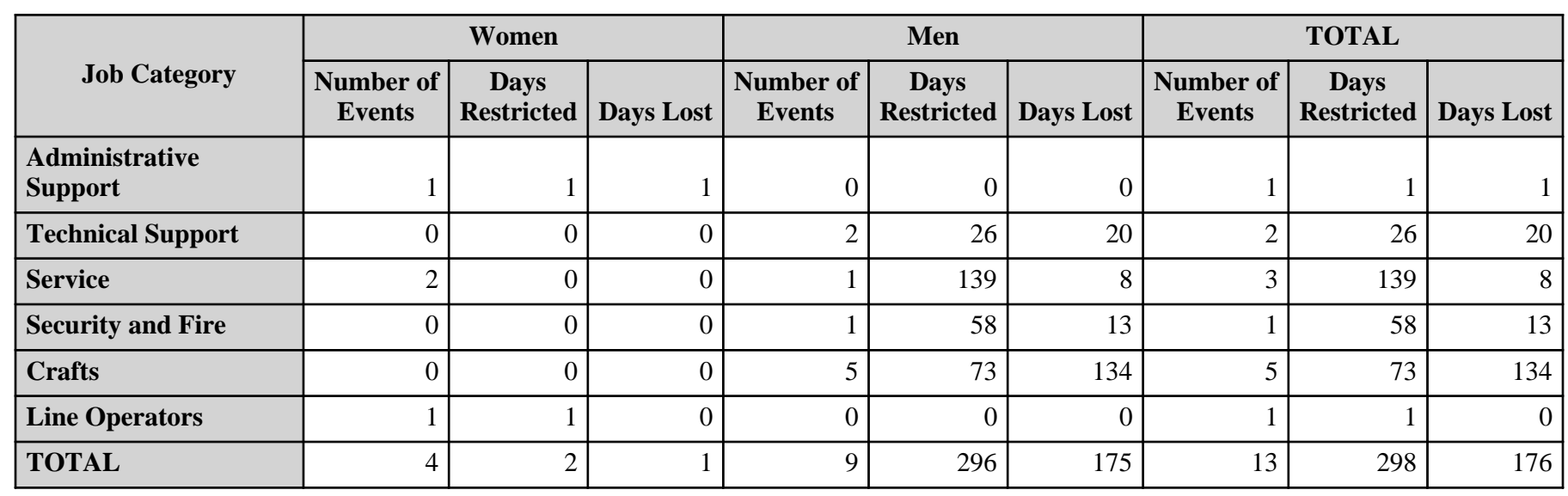

*Only those job categories with at least one OSHA event appear in this table. 


\section{Kansas City Plant 2008}

OSHA Data

Appendix O. Number of Diagnoses in Each Diagnostic Category by Gender and Age*

\begin{tabular}{|c|c|c|c|c|c|c|c|c|}
\hline & & \multicolumn{3}{|c|}{ Women } & \multicolumn{3}{|c|}{ Men } & \multirow[b]{3}{*}{ TOTAL } \\
\hline & & \multicolumn{2}{|c|}{ Age Group } & \multirow[b]{2}{*}{ TOTAL } & \multicolumn{2}{|c|}{ Age Group } & \multirow[b]{2}{*}{ TOTAL } & \\
\hline & & $40-49$ & $50+$ & & $40-49$ & $50+$ & & \\
\hline Diagnostic Category & ICD-9-CM code & & & & & & & \\
\hline MALIGNANT NEOPLASMS & $140-208,230-234$ & 0 & 0 & 0 & 0 & 1 & 1 & 1 \\
\hline -Respiratory \& Intrathoracic & $160-165$ & 0 & 0 & 0 & 0 & 1 & 1 & 1 \\
\hline NERVOUS SYSTEM (NS) \& SENSE ORGANS & 320-389 & 0 & 3 & 3 & 0 & 0 & 0 & 3 \\
\hline -Disorders of Peripheral NS & $350-359$ & 0 & 3 & 3 & 0 & 0 & 0 & 3 \\
\hline RESPIRATORY SYSTEM & $460-519$ & 1 & 0 & 1 & 0 & 0 & 0 & 1 \\
\hline -Lung Dis from External Agents & $500-508$ & 1 & 0 & 1 & 0 & 0 & 0 & 1 \\
\hline DIGESTIVE SYSTEM & $520-579$ & 0 & 0 & 0 & 1 & 0 & 1 & 1 \\
\hline -Hernia & $550-553$ & 0 & 0 & 0 & 1 & 0 & 1 & 1 \\
\hline $\begin{array}{l}\text { MUSCULOSKELETAL \& CONNECTIVE } \\
\text { TISSUE }\end{array}$ & $710-739$ & 2 & 3 & 5 & 0 & 9 & 9 & 14 \\
\hline -Arthropathies & $710-719$ & 1 & 2 & 3 & 0 & 6 & 6 & 9 \\
\hline -Rheumatism, Excluding Back & $725-729$ & 1 & 1 & 2 & 0 & 3 & 3 & 5 \\
\hline $\begin{array}{l}\text { SYMPTOMS, SIGNS, \& ILL-DEFINED } \\
\text { CONDITIONS }\end{array}$ & $780-799$ & 0 & 0 & 0 & 2 & 0 & 2 & 2 \\
\hline -Symptoms & $780-789$ & 0 & 0 & 0 & 2 & 0 & 2 & 2 \\
\hline INJURY \& POISONING & 800-999 & 1 & 1 & 2 & 0 & 5 & 5 & 7 \\
\hline -Sprains \& Strains - Other & $840-845,848$ & 0 & 0 & 0 & 0 & 1 & 1 & 1 \\
\hline -Open Wound - Upper Limb & $880-887$ & 0 & 1 & 1 & 0 & 1 & 1 & 2 \\
\hline -Burns & $940-949$ & 0 & 0 & 0 & 0 & 1 & 1 & 1 \\
\hline -Complications \& Unspecified Injuries & 958-959 & 1 & 0 & 1 & 0 & 2 & 2 & 3 \\
\hline
\end{tabular}

\begin{tabular}{|c|c|c|c|c|c|c|c|}
\hline & \multicolumn{3}{|c|}{ Women } & \multicolumn{3}{|c|}{ Men } & \multirow[b]{3}{*}{ TOTAL } \\
\hline & \multicolumn{2}{|c|}{ Age Group } & \multirow[b]{2}{*}{ TOTAL } & \multicolumn{2}{|c|}{ Age Group } & \multirow[b]{2}{*}{ TOTAL } & \\
\hline & $40-49$ & $50+$ & & $40-49$ & $50+$ & & \\
\hline Diagnostic Category & \multirow[b]{2}{*}{4} & \multirow[b]{2}{*}{7} & \multirow[b]{2}{*}{11} & \multirow[b]{2}{*}{3} & \multirow[b]{2}{*}{15} & \multirow[b]{2}{*}{18} & \multirow[b]{2}{*}{29} \\
\hline Total & & & & & & & \\
\hline
\end{tabular}

*Only those diagnostic categories and gender/age combinations with at least one OSHA event appear in this table. 


\section{Kansas City Plant 2008}

OSHA Data

Appendix P. Number of Workdays Lost or with Restricted Activity in Each Diagnostic Category by Gender and Age*

\begin{tabular}{|c|c|c|c|c|c|}
\hline & & \multicolumn{4}{|c|}{ Women } \\
\hline & & \multicolumn{4}{|c|}{ Age Group } \\
\hline & & \multicolumn{2}{|c|}{$40-49$} & \multicolumn{2}{|c|}{$50+$} \\
\hline & & \begin{tabular}{|c|} 
Days \\
Restricted
\end{tabular} & Days Lost & $\begin{array}{c}\text { Days } \\
\text { Restricted }\end{array}$ & Days Lost \\
\hline Diagnostic Category & ICD-9-CM Codes & \multirow[b]{2}{*}{0} & \multirow[b]{2}{*}{0} & \multirow[b]{2}{*}{1} & \multirow[b]{2}{*}{1} \\
\hline -Disorders of Peripheral NS & $350-359$ & & & & \\
\hline -Lung Dis from External Agents & $500-508$ & 0 & 0 & 0 & 0 \\
\hline -Arthropathies & $710-719$ & 1 & 0 & 1 & 1 \\
\hline -Rheumatism, Excluding Back & $725-729$ & 1 & 0 & 1 & 1 \\
\hline -Open Wound - Upper Limb & $880-887$ & 0 & 0 & 0 & 0 \\
\hline $\begin{array}{l}\text {-Complications \& Unspecified } \\
\text { Injuries }\end{array}$ & 958-959 & 1 & 0 & 0 & 0 \\
\hline
\end{tabular}

\begin{tabular}{|c|c|c|c|c|c|}
\hline & & \multicolumn{4}{|c|}{ Men } \\
\hline & & \multicolumn{4}{|c|}{ Age Group } \\
\hline & & \multicolumn{2}{|c|}{$40-49$} & \multicolumn{2}{|c|}{$50+$} \\
\hline & & \begin{tabular}{|c|} 
Days \\
Restricted
\end{tabular} & Days Lost & \begin{tabular}{|c|} 
Days \\
Restricted
\end{tabular} & Days Lost \\
\hline Diagnostic Category & ICD-9-CM Codes & \multirow[b]{2}{*}{0} & \multirow[b]{2}{*}{0} & \multirow[b]{2}{*}{1} & \multirow[b]{2}{*}{134} \\
\hline -Respiratory \& Intrathoracic & $160-165$ & & & & \\
\hline -Hernia & $550-553$ & 26 & 20 & 0 & 0 \\
\hline -Arthropathies & $710-719$ & 0 & 0 & 130 & 13 \\
\hline -Rheumatism, Excluding Back & 725-729 & 0 & 0 & 211 & 8 \\
\hline -Symptoms & $780-789$ & 26 & 20 & 0 & 0 \\
\hline -Sprains \& Strains - Other & $840-845,848$ & 0 & 0 & 58 & 13 \\
\hline -Open Wound - Upper Limb & $880-887$ & 0 & 0 & 0 & 0 \\
\hline -Burns & 940-949 & 0 & 0 & 0 & 0 \\
\hline $\begin{array}{l}\text {-Complications \& Unspecified } \\
\text { Injuries }\end{array}$ & 958-959 & 0 & 0 & 139 & 8 \\
\hline
\end{tabular}

*OSHA events with >1 ICD-9-CM code in the same diagnostic category were counted only once. Only those diagnostic categories and gender/age combinations with at least one occurrence appear in this table. 
Kansas City Plant 2008

OSHA Data

Appendix Q. Number of Occurrences in Each Accident Category by Gender and Age*

\begin{tabular}{|c|c|c|c|c|c|c|c|c|}
\hline & & \multicolumn{3}{|c|}{ Women } & \multicolumn{3}{|c|}{ Men } & \multirow[b]{3}{*}{ TOTAL } \\
\hline & & \multicolumn{2}{|c|}{ Age Group } & \multirow[b]{2}{*}{ TOTAL } & \multicolumn{2}{|c|}{ Age Group } & \multirow[b]{2}{*}{ TOTAL } & \\
\hline & & $40-49$ & $50+$ & & $40-49$ & $50+$ & & \\
\hline Type of Accident & E Codes & & & & & & & \\
\hline Falls & E880-E888 & 0 & 0 & 0 & 0 & 2 & 2 & 2 \\
\hline Other Accidents & E916-E928 & 1 & 2 & 3 & 1 & 5 & 6 & 9 \\
\hline
\end{tabular}

*Only those accident types and gender/age combinations with at least one occurrence appear in this table. 
Kansas City Plant 2008

OSHA Data

Appendix R. Number of Workdays Lost or with Restricted Activity in Each Accident Category by Gender and Age*

\begin{tabular}{|c|c|c|c|c|c|}
\hline & & \multicolumn{4}{|c|}{ Women } \\
\hline & & \multicolumn{4}{|c|}{ Age Group } \\
\hline & & \multicolumn{2}{|c|}{$40-49$} & \multicolumn{2}{|c|}{$50+$} \\
\hline & & $\begin{array}{c}\text { Days } \\
\text { Restricted }\end{array}$ & Days Lost & $\begin{array}{c}\text { Days } \\
\text { Restricted }\end{array}$ & Days Lost \\
\hline Type of Accident & E Codes & \multirow[b]{2}{*}{1} & \multirow[b]{2}{*}{0} & \multirow[b]{2}{*}{1} & \multirow[b]{2}{*}{1} \\
\hline Other Accidents & E916-E928 & & & & \\
\hline
\end{tabular}

\begin{tabular}{|c|c|c|c|c|c|}
\hline & & \multicolumn{4}{|c|}{ Men } \\
\hline & & \multicolumn{4}{|c|}{ Age Group } \\
\hline & & \multicolumn{2}{|c|}{$40-49$} & \multicolumn{2}{|c|}{$50+$} \\
\hline & & $\begin{array}{c}\text { Days } \\
\text { Restricted }\end{array}$ & Days Lost & \begin{tabular}{c|} 
Days \\
Restricted
\end{tabular} & Days Lost \\
\hline Type of Accident & E Codes & \multirow[b]{2}{*}{0} & \multirow[b]{2}{*}{0} & \multirow[b]{2}{*}{0} & \multirow[b]{2}{*}{0} \\
\hline Falls & E880-E888 & & & & \\
\hline Other Accidents & \begin{tabular}{|l|} 
E916-E928 \\
\end{tabular} & 26 & 20 & 269 & 21 \\
\hline
\end{tabular}

*OSHA events with $>1 \mathrm{E}$ code in the same accident type were counted only once. Only those accident types and gender/age combinations with at least one occurrence appear in this table. 


\section{Kansas City Plant 2008}

OSHA Data

Appendix S. Number of Diagnoses in Each Diagnostic Category by Gender and Job Category*

\begin{tabular}{|c|c|c|c|c|c|}
\hline & & \multicolumn{4}{|c|}{ Women } \\
\hline & & \multicolumn{3}{|c|}{ Job Category } & \multirow[b]{2}{*}{ TOTAL } \\
\hline & & $\begin{array}{c}\text { Administrative } \\
\text { Support }\end{array}$ & Service & Line Operators & \\
\hline Diagnostic Category & ICD-9-CM Code & & & & \\
\hline $\begin{array}{l}\text { NERVOUS SYSTEM } \\
\text { (NS) \& SENSE ORGANS }\end{array}$ & $320-389$ & 3 & 0 & 0 & 3 \\
\hline $\begin{array}{l}\text {-Disorders of Peripheral } \\
\text { NS }\end{array}$ & $350-359$ & 3 & 0 & 0 & 3 \\
\hline $\begin{array}{l}\text { RESPIRATORY } \\
\text { SYSTEM }\end{array}$ & $460-519$ & 0 & 1 & 0 & 1 \\
\hline $\begin{array}{l}\text {-Lung Dis from External } \\
\text { Agents }\end{array}$ & $500-508$ & 0 & 1 & 0 & 1 \\
\hline $\begin{array}{l}\text { MUSCULOSKELETAL } \\
\text { \& CONNECTIVE } \\
\text { TISSUE }\end{array}$ & $710-739$ & 3 & 0 & 2 & 5 \\
\hline -Arthropathies & $710-719$ & 2 & 0 & 1 & 3 \\
\hline $\begin{array}{l}\text {-Rheumatism, Excluding } \\
\text { Back }\end{array}$ & $725-729$ & 1 & 0 & 1 & 2 \\
\hline INJURY \& POISONING & 800-999 & 0 & 1 & 1 & 2 \\
\hline $\begin{array}{l}\text {-Open Wound - Upper } \\
\text { Limb }\end{array}$ & $880-887$ & 0 & 1 & 0 & 1 \\
\hline $\begin{array}{l}\text {-Complications \& } \\
\text { Unspecified Injuries }\end{array}$ & 958-959 & 0 & 0 & 1 & 1 \\
\hline
\end{tabular}

\begin{tabular}{|c|c|c|c|c|}
\hline & \multicolumn{4}{|c|}{ Women } \\
\hline & \multicolumn{3}{|c|}{ Job Category } & \multirow[b]{2}{*}{ TOTAL } \\
\hline & $\begin{array}{c}\text { Administrative } \\
\text { Support }\end{array}$ & Service & Line Operators & \\
\hline Diagnostic Category & \multirow[b]{2}{*}{6} & \multirow[b]{2}{*}{2} & \multirow[b]{2}{*}{3} & \multirow[b]{2}{*}{11} \\
\hline Total & & & & \\
\hline
\end{tabular}

\begin{tabular}{|c|c|c|c|c|c|c|}
\hline & & \multicolumn{5}{|c|}{ Men } \\
\hline & & \multicolumn{4}{|c|}{ Job Category } & \multirow[b]{2}{*}{ TOTAL } \\
\hline & & Technical Support & Service & Security and Fire & Crafts & \\
\hline Diagnostic Category & ICD-9-CM Code & & & & & \\
\hline $\begin{array}{l}\text { MALIGNANT } \\
\text { NEOPLASMS }\end{array}$ & $140-208,230-234$ & 0 & 0 & 0 & 1 & 1 \\
\hline $\begin{array}{l}\text {-Respiratory \& } \\
\text { Intrathoracic }\end{array}$ & $160-165$ & 0 & 0 & 0 & 1 & 1 \\
\hline DIGESTIVE SYSTEM & $520-579$ & 1 & 0 & 0 & 0 & 1 \\
\hline -Hernia & $550-553$ & 1 & 0 & 0 & 0 & 1 \\
\hline $\begin{array}{l}\text { MUSCULOSKELETAL } \\
\text { \& CONNECTIVE } \\
\text { TISSUE }\end{array}$ & $710-739$ & 1 & 1 & 1 & 6 & 9 \\
\hline -Arthropathies & $710-719$ & 1 & 0 & 1 & 4 & 6 \\
\hline
\end{tabular}

(Continued)

*Only those diagnostic categories and gender/job category combinations with at least one occurrence appear in this table. 


\section{Kansas City Plant 2008}

OSHA Data

Appendix S. Number of Diagnoses in Each Diagnostic Category by Gender and Job Category*

\begin{tabular}{|c|c|c|c|c|c|c|}
\hline & & \multicolumn{5}{|c|}{ Men } \\
\hline & & \multicolumn{4}{|c|}{ Job Category } & \multirow[b]{2}{*}{ TOTAL } \\
\hline & & Technical Support & Service & Security and Fire & Crafts & \\
\hline Diagnostic Category & ICD-9-CM Code & & & & & \\
\hline $\begin{array}{l}\text {-Rheumatism, Excluding } \\
\text { Back }\end{array}$ & $725-729$ & 0 & 1 & 0 & 2 & 3 \\
\hline $\begin{array}{l}\text { SYMPTOMS, } \\
\text { SIGNS, \& ILL-DEFINED } \\
\text { CONDITIONS }\end{array}$ & $780-799$ & 2 & 0 & 0 & 0 & 2 \\
\hline -Symptoms & $780-789$ & 2 & 0 & 0 & 0 & 2 \\
\hline INJURY \& POISONING & $800-999$ & 0 & 1 & 1 & 3 & 5 \\
\hline $\begin{array}{l}\text {-Sprains \& Strains - } \\
\text { Other }\end{array}$ & $840-845,848$ & 0 & 0 & 1 & 0 & 1 \\
\hline $\begin{array}{l}\text {-Open Wound - Upper } \\
\text { Limb }\end{array}$ & $880-887$ & 0 & 0 & 0 & 1 & 1 \\
\hline -Burns & 940-949 & 0 & 0 & 0 & 1 & 1 \\
\hline $\begin{array}{l}\text {-Complications \& } \\
\text { Unspecified Injuries }\end{array}$ & 958-959 & 0 & 1 & 0 & 1 & 2 \\
\hline
\end{tabular}

\begin{tabular}{|c|c|c|c|c|c|}
\hline & \multicolumn{5}{|c|}{ Men } \\
\hline & \multicolumn{4}{|c|}{ Job Category } & \multirow[b]{2}{*}{ TOTAL } \\
\hline & Technical Support & Service & Security and Fire & Crafts & \\
\hline Diagnostic Category & \multirow[b]{2}{*}{4} & \multirow[b]{2}{*}{2} & \multirow[b]{2}{*}{2} & \multirow[b]{2}{*}{10} & \multirow[b]{2}{*}{18} \\
\hline Total & & & & & \\
\hline
\end{tabular}

*Only those diagnostic categories and gender/job category combinations with at least one occurrence appear in this table. 


\section{Kansas City Plant 2008}

OSHA Data

Appendix T. Number of Workdays Lost or with Restricted Activity in Each Diagnostic Category by Gender and Job Category*

\begin{tabular}{|c|c|c|c|c|c|c|c|}
\hline & & \multicolumn{6}{|c|}{ Women } \\
\hline & & \multicolumn{6}{|c|}{ Job Category } \\
\hline & & \multicolumn{2}{|c|}{$\begin{array}{l}\text { Administrative } \\
\text { Support }\end{array}$} & \multicolumn{2}{|c|}{ Service } & \multicolumn{2}{|c|}{ Line Operators } \\
\hline & & $\begin{array}{c}\text { Days } \\
\text { Restricted }\end{array}$ & Days Lost & $\begin{array}{c}\text { Days } \\
\text { Restricted }\end{array}$ & Days Lost & $\begin{array}{c}\text { Days } \\
\text { Restricted }\end{array}$ & Days Lost \\
\hline Diagnostic Category & ICD-9-CM Codes & & & & & & \\
\hline -Disorders of Peripheral NS & $350-359$ & 1 & 1 & 0 & 0 & 0 & 0 \\
\hline -Lung Dis from External Agents & $500-508$ & 0 & 0 & 0 & 0 & 0 & 0 \\
\hline -Arthropathies & $710-719$ & 1 & 1 & 0 & 0 & 1 & 0 \\
\hline -Rheumatism, Excluding Back & 725-729 & 1 & 1 & 0 & 0 & 1 & 0 \\
\hline -Open Wound - Upper Limb & $880-887$ & 0 & 0 & 0 & 0 & 0 & 0 \\
\hline $\begin{array}{l}\text {-Complications \& Unspecified } \\
\text { Injuries }\end{array}$ & 958-959 & 0 & 0 & 0 & 0 & 1 & 0 \\
\hline
\end{tabular}

\begin{tabular}{|c|c|c|c|c|c|c|c|c|c|}
\hline & & \multicolumn{8}{|c|}{ Men } \\
\hline & & \multicolumn{8}{|c|}{ Job Category } \\
\hline & & \multicolumn{2}{|c|}{ Technical Support } & \multicolumn{2}{|c|}{ Service } & \multicolumn{2}{|c|}{ Security and Fire } & \multicolumn{2}{|c|}{ Crafts } \\
\hline & & $\begin{array}{c}\text { Days } \\
\text { Restricted }\end{array}$ & $\begin{array}{l}\text { Days } \\
\text { Lost }\end{array}$ & $\begin{array}{c}\text { Days } \\
\text { Restricted }\end{array}$ & $\begin{array}{l}\text { Days } \\
\text { Lost }\end{array}$ & $\begin{array}{c}\text { Days } \\
\text { Restricted }\end{array}$ & $\begin{array}{c}\text { Days } \\
\text { Lost }\end{array}$ & $\begin{array}{c}\text { Days } \\
\text { Restricted }\end{array}$ & $\begin{array}{l}\text { Days } \\
\text { Lost }\end{array}$ \\
\hline Diagnostic Category & ICD-9-CM Codes & & & & & & & & \\
\hline -Respiratory \& Intrathoracic & $160-165$ & 0 & 0 & 0 & 0 & 0 & 0 & 1 & 134 \\
\hline -Hernia & $550-553$ & 26 & 20 & 0 & 0 & 0 & 0 & 0 & 0 \\
\hline -Arthropathies & $710-719$ & 0 & 0 & 0 & 0 & 58 & 13 & 72 & 0 \\
\hline -Rheumatism, Excluding Back & 725-729 & 0 & 0 & 139 & 8 & 0 & 0 & 72 & 0 \\
\hline -Symptoms & $780-789$ & 26 & 20 & 0 & 0 & 0 & 0 & 0 & 0 \\
\hline -Sprains \& Strains - Other & $840-845,848$ & 0 & 0 & 0 & 0 & 58 & 13 & 0 & 0 \\
\hline -Open Wound - Upper Limb & $880-887$ & 0 & 0 & 0 & 0 & 0 & 0 & 0 & 0 \\
\hline -Burns & $940-949$ & 0 & 0 & 0 & 0 & 0 & 0 & 0 & 0 \\
\hline $\begin{array}{l}\text {-Complications \& Unspecified } \\
\text { Injuries }\end{array}$ & 958-959 & 0 & 0 & 139 & 8 & 0 & 0 & 0 & 0 \\
\hline
\end{tabular}

*OSHA events with >1 ICD-9-CM code in the same diagnostic category were counted only once. Only those diagnostic categories and gender/job category combinations with at least one occurrence appear in this table. 
Kansas City Plant 2008

OSHA Data

Appendix U. Number of Occurrences in Each Accident Category by Gender and Job Category*

\begin{tabular}{|c|c|c|c|c|c|}
\hline & & \multicolumn{4}{|c|}{ Women } \\
\hline & & \multicolumn{3}{|c|}{ Job Category } & \multirow[b]{2}{*}{ TOTAL } \\
\hline & & $\begin{array}{l}\text { Administrative } \\
\text { Support }\end{array}$ & Service & $\begin{array}{c}\text { Line } \\
\text { Operators }\end{array}$ & \\
\hline Type of Accident & E CODES & \multirow[b]{2}{*}{1} & \multirow[b]{2}{*}{1} & \multirow[b]{2}{*}{1} & \multirow[b]{2}{*}{3} \\
\hline Other Accidents & E916-E928 & & & & \\
\hline
\end{tabular}

\begin{tabular}{|c|c|c|c|c|c|c|}
\hline & & \multicolumn{5}{|c|}{ Men } \\
\hline & & \multicolumn{4}{|c|}{ Job Category } & \multirow[b]{2}{*}{ TOTAL } \\
\hline & & $\begin{array}{l}\text { Technical } \\
\text { Support }\end{array}$ & Service & $\begin{array}{c}\text { Security and } \\
\text { Fire }\end{array}$ & Crafts & \\
\hline Type of Accident & E CODES & \multirow[b]{2}{*}{1} & \multirow[b]{2}{*}{0} & \multirow[b]{2}{*}{0} & \multirow[b]{2}{*}{1} & \multirow[b]{2}{*}{2} \\
\hline Falls & E880-E888 & & & & & \\
\hline Other Accidents & E916-E928 & 1 & 1 & 1 & 3 & 6 \\
\hline
\end{tabular}

*Only those accident types and gender/job category combinations with at least one occurrence appear in this table. 


\section{Kansas City Plant 2008}

OSHA Data

Appendix V. Number of Workdays Lost or with Restricted Activity in Each Accident Category by Gender and Job Category*

\begin{tabular}{|c|c|c|c|c|c|c|c|}
\hline & & \multicolumn{6}{|c|}{ Women } \\
\hline & & \multicolumn{6}{|c|}{ Job Category } \\
\hline & & \multicolumn{2}{|c|}{$\begin{array}{c}\text { Administrative } \\
\text { Support }\end{array}$} & \multicolumn{2}{|c|}{ Service } & \multicolumn{2}{|c|}{ Line Operators } \\
\hline & & $\begin{array}{c}\text { Days } \\
\text { Restricted }\end{array}$ & Days Lost & $\begin{array}{c}\text { Days } \\
\text { Restricted }\end{array}$ & Days Lost & $\begin{array}{c}\text { Days } \\
\text { Restricted }\end{array}$ & Days Lost \\
\hline Type of Accident & E Codes & \multirow[b]{2}{*}{1} & \multirow[b]{2}{*}{1} & \multirow[b]{2}{*}{0} & \multirow[b]{2}{*}{0} & \multirow[b]{2}{*}{1} & \multirow[b]{2}{*}{0} \\
\hline Other Accidents & E916-E928 & & & & & & \\
\hline
\end{tabular}

\begin{tabular}{|c|c|c|c|c|c|c|c|c|c|}
\hline & & \multicolumn{8}{|c|}{ Men } \\
\hline & & \multicolumn{8}{|c|}{ Job Category } \\
\hline & & \multicolumn{2}{|c|}{ Technical Support } & \multicolumn{2}{|c|}{ Service } & \multicolumn{2}{|c|}{ Security and Fire } & \multicolumn{2}{|c|}{ Crafts } \\
\hline & & $\begin{array}{c}\text { Days } \\
\text { Restricted }\end{array}$ & $\begin{array}{l}\text { Days } \\
\text { Lost }\end{array}$ & $\begin{array}{c}\text { Days } \\
\text { Restricted }\end{array}$ & $\begin{array}{l}\text { Days } \\
\text { Lost }\end{array}$ & $\begin{array}{c}\text { Days } \\
\text { Restricted }\end{array}$ & $\begin{array}{l}\text { Days } \\
\text { Lost }\end{array}$ & $\begin{array}{c}\text { Days } \\
\text { Restricted }\end{array}$ & $\begin{array}{l}\text { Days } \\
\text { Lost }\end{array}$ \\
\hline Type of Accident & E Codes & \multirow[b]{2}{*}{0} & \multirow[b]{2}{*}{0} & \multirow[b]{2}{*}{0} & \multirow[b]{2}{*}{0} & \multirow[b]{2}{*}{0} & \multirow[b]{2}{*}{0} & \multirow[b]{2}{*}{0} & \multirow[b]{2}{*}{0} \\
\hline Falls & E880-E888 & & & & & & & & \\
\hline Other Accidents & E916-E928 & 26 & 20 & 139 & 8 & 58 & 13 & 72 & 0 \\
\hline
\end{tabular}

*OSHA events with >1 E code in the same accident type were counted only once. Only those accident types and gender/job category combinations with at least one occurrence appear in this table. 
Kansas City Plant 2008

OSHA Data

Appendix W. Age-Adjusted OSHA Illness and Injury Rates by Diagnostic Category*

Part 1. Men

\begin{tabular}{|c|c|c|c|c|c|}
\hline & & $\begin{array}{l}\text { Number of } \\
\text { Diagnoses }\end{array}$ & $\begin{array}{c}\text { Age-Adjusted } \\
\text { Rate per } 1,000 * *\end{array}$ & $\begin{array}{c}\text { Lower } 95 \% \\
\text { Confidence } \\
\text { Limit per 1,000 }\end{array}$ & $\begin{array}{c}\text { Upper } 95 \% \\
\text { Confidence } \\
\text { Limit per } 1,000\end{array}$ \\
\hline Diagnostic Category & ICD-9-CM Code & \multirow[b]{2}{*}{1} & \multirow[b]{2}{*}{0.4} & \multirow[b]{2}{*}{0.1} & \multirow[b]{2}{*}{2.9} \\
\hline MALIGNANT NEOPLASMS & $140-208,230-234$ & & & & \\
\hline -Respiratory \& Intrathoracic & $160-165$ & 1 & 0.4 & 0.1 & 2.9 \\
\hline DIGESTIVE SYSTEM & $520-579$ & 1 & 0.4 & 0.1 & 3.0 \\
\hline -Hernia & $550-553$ & 1 & 0.4 & 0.1 & 3.0 \\
\hline $\begin{array}{l}\text { MUSCULOSKELETAL \& CONNECTIVE } \\
\text { TISSUE }\end{array}$ & $710-739$ & 9 & 1.9 & 1.0 & 3.6 \\
\hline -Arthropathies & 710-719 & 6 & 1.3 & 0.6 & 2.8 \\
\hline -Rheumatism, Excluding Back & 725-729 & 3 & 0.6 & 0.2 & 1.9 \\
\hline $\begin{array}{l}\text { SYMPTOMS, SIGNS, \& ILL-DEFINED } \\
\text { CONDITIONS }\end{array}$ & 780-799 & 2 & 0.8 & 0.2 & 3.4 \\
\hline -Symptoms & 780-789 & 2 & 0.8 & 0.2 & 3.4 \\
\hline INJURY \& POISONING & $800-999$ & 5 & 1.0 & 0.4 & 2.5 \\
\hline -Sprains \& Strains - Other & $840-845,848$ & 1 & 0.2 & 0.0 & 1.5 \\
\hline -Open Wound - Upper Limb & $880-887$ & 1 & 0.2 & 0.0 & 1.5 \\
\hline -Burns & $940-949$ & 1 & 0.2 & 0.0 & 1.5 \\
\hline -Complications of Surgical/Medical Care & 996-999 & 2 & 0.4 & 0.1 & 1.7 \\
\hline Total & & 18 & 4.6 & 2.8 & 7.5 \\
\hline
\end{tabular}

* Only those diagnostic categories with at least one occurrence appear in this table.

**Standardized to age distribution of 2000 U.S. population. 
Kansas City Plant 2008

OSHA Data

Appendix W. Age-Adjusted OSHA Illness and Injury Rates by Diagnostic Category*

Part 2. Women

\begin{tabular}{|c|c|c|c|c|c|}
\hline & & $\begin{array}{l}\text { Number of } \\
\text { Diagnoses }\end{array}$ & $\begin{array}{c}\text { Age-Adjusted } \\
\text { Rate per } 1,000 * *\end{array}$ & $\begin{array}{c}\text { Lower } 95 \% \\
\text { Confidence } \\
\text { Limit per 1,000 }\end{array}$ & $\begin{array}{c}\text { Upper } 95 \% \\
\text { Confidence } \\
\text { Limit per 1,000 }\end{array}$ \\
\hline Diagnostic Category & ICD-9-CM Code & \multirow[b]{2}{*}{3} & \multirow[b]{2}{*}{4.5} & \multirow[b]{2}{*}{1.4} & \multirow[b]{2}{*}{13.9} \\
\hline NERVOUS SYSTEM (NS) \& SENSE ORGANS & 320-389 & & & & \\
\hline -Disorders of Peripheral NS & $350-359$ & 3 & 4.5 & 1.4 & 13.9 \\
\hline RESPIRATORY SYSTEM & $460-519$ & 1 & 1.3 & 0.2 & 8.9 \\
\hline -Lung Dis from External Agents & $500-508$ & 1 & 1.3 & 0.2 & 8.9 \\
\hline $\begin{array}{l}\text { MUSCULOSKELETAL \& CONNECTIVE } \\
\text { TISSUE }\end{array}$ & 710-739 & 5 & 7.0 & 2.9 & 16.9 \\
\hline -Arthropathies & $710-719$ & 3 & 4.2 & 1.4 & 13.2 \\
\hline -Rheumatism, Excluding Back & 725-729 & 2 & 2.7 & 0.7 & 11.0 \\
\hline INJURY \& POISONING & $800-999$ & 2 & 1.9 & 0.4 & 8.1 \\
\hline -Open Wound - Upper Limb & $880-887$ & 1 & 0.6 & 0.1 & 4.4 \\
\hline -Complications of Surgical/Medical Care & 996-999 & 1 & 1.3 & 0.2 & 8.9 \\
\hline Total & & 11 & 14.6 & 8.0 & 26.6 \\
\hline
\end{tabular}

* Only those diagnostic categories with at least one occurrence appear in this table.

**Standardized to age distribution of 2000 U.S. population. 


\section{Kansas City Plant 2008}

OSHA Data

Appendix W. Age-Adjusted OSHA Illness and Injury Rates by Diagnostic Category*

Part 3. Men and Women

\begin{tabular}{|c|c|c|c|c|c|}
\hline & & $\begin{array}{l}\text { Number of } \\
\text { Diagnoses }\end{array}$ & $\begin{array}{c}\text { Age-Adjusted } \\
\text { Rate per 1,000** }\end{array}$ & $\begin{array}{c}\text { Lower } 95 \% \\
\text { Confidence } \\
\text { Limit per } 1,000\end{array}$ & $\begin{array}{c}\text { Upper 95\% } \\
\text { Confidence } \\
\text { Limit per 1,000 }\end{array}$ \\
\hline Diagnostic Category & ICD-9-CM Code & \multirow[b]{2}{*}{1} & \multirow[b]{2}{*}{0.3} & \multirow[b]{2}{*}{0.0} & \multirow[b]{2}{*}{2.3} \\
\hline MALIGNANT NEOPLASMS & $140-208,230-234$ & & & & \\
\hline -Respiratory \& Intrathoracic & $160-165$ & 1 & 0.3 & 0.0 & 2.3 \\
\hline NERVOUS SYSTEM (NS) \& SENSE ORGANS & 320-389 & 3 & 1.0 & 0.3 & 3.0 \\
\hline -Disorders of Peripheral NS & $350-359$ & 3 & 1.0 & 0.3 & 3.0 \\
\hline RESPIRATORY SYSTEM & $460-519$ & 1 & 0.3 & 0.0 & 2.2 \\
\hline -Lung Dis from External Agents & $500-508$ & 1 & 0.3 & 0.0 & 2.2 \\
\hline DIGESTIVE SYSTEM & $520-579$ & 1 & 0.3 & 0.0 & 2.2 \\
\hline -Hernia & $550-553$ & 1 & 0.3 & 0.0 & 2.2 \\
\hline $\begin{array}{l}\text { MUSCULOSKELETAL \& CONNECTIVE } \\
\text { TISSUE }\end{array}$ & 710-739 & 14 & 3.0 & 1.7 & 5.3 \\
\hline -Arthropathies & $710-719$ & 9 & 1.9 & 1.0 & 3.8 \\
\hline -Rheumatism, Excluding Back & $725-729$ & 5 & 1.1 & 0.4 & 2.8 \\
\hline $\begin{array}{l}\text { SYMPTOMS, SIGNS, \& ILL-DEFINED } \\
\text { CONDITIONS }\end{array}$ & $780-799$ & 2 & 0.6 & 0.2 & 2.5 \\
\hline -Symptoms & $780-789$ & 2 & 0.6 & 0.2 & 2.5 \\
\hline INJURY \& POISONING & $800-999$ & 7 & 1.3 & 0.6 & 2.7 \\
\hline -Sprains \& Strains - Other & $840-845,848$ & 1 & 0.2 & 0.0 & 1.1 \\
\hline -Open Wound - Upper Limb & $880-887$ & 2 & 0.3 & 0.1 & 1.3 \\
\hline -Burns & $940-949$ & 1 & 0.2 & 0.0 & 1.1 \\
\hline -Complications of Surgical/Medical Care & 996-999 & 3 & 0.6 & 0.2 & 2.1 \\
\hline Total & & 29 & 6.9 & 4.7 & 10.1 \\
\hline
\end{tabular}

* Only those diagnostic categories with at least one occurrence appear in this table.

**Standardized to age distribution of 2000 U.S. population. 